\title{
RAISING THE BAR IN CLINICAL PERFECTIONISM: AN EVALUATION IN THE DOMAIN OF WEIGHT LOSS
}

by

Kirstyn L. Krause

Bachelor of Arts (Honours) in Psychology, York University, Toronto, Ontario, 2013

A thesis
presented to Ryerson University

in partial fulfillment of the requirements for the degree of Master of Arts

in the Program of Psychology

Toronto, Ontario, Canada, 2016

(C) (Kirstyn L. Krause) 2016 


\section{AUTHOR'S DECLARATION FOR ELECTRONIC SUBMISSION OF A THESIS}

I hereby declare that I am the sole author of this thesis. This is a true copy of the thesis, including any required final revisions, as accepted by my examiners.

I authorize Ryerson University to lend this thesis to other institutions or individuals for the purpose of scholarly research

I further authorize Ryerson University to reproduce this thesis by photocopying or by other means, in total or in part, at the request of other institutions or individuals for the purpose of scholarly research.

I understand that my thesis may be made electronically available to the public. 


\title{
Raising the Bar in Clinical Perfectionism: \\ An Evaluation in the Domain of Weight Loss
}

Master of Arts, 2016

Kirstyn L. Krause

Psychology

Ryerson University

\begin{abstract}
Clinical perfectionists who successfully meet a salient, personally demanding, and selfimposed standard are thought to feel dissatisfied with success, reappraise the standard as insufficient, and raise the bar by setting more challenging goals (Shafran et al., 2002). Past studies evaluate raising the bar using tasks that are not salient, personally demanding, or selfimposed. The present study examined raising the bar in the salient domain of weight loss. Overweight participants completed a 2-week food and exercise diary during which they set personal weight goals. Raising the bar was measured by calculating the difference between weight goals at separate time points. The General Goal-setting Questionnaire (Krause et al., 2016) measured (1) standard dissatisfaction and reappraisal, and (2) the tendency to raise the bar. Results revealed that clinical perfectionism significantly predicted standard dissatisfaction and reappraisal, but not the tendency to raise the bar or an increase in weight goals. Implications are discussed.
\end{abstract}




\section{Acknowledgements}

First and foremost, I would like to express my gratitude to my supervisor, Dr. Martin Antony, for his continued support and guidance throughout the entire process of my Masters thesis. Thank you for your constant availability to answer questions and address concerns, for encouraging me to think through the project in a critical and creative manner, and for challenging me to pursue excellence in graduate research.

I would also like to thank Dr. Stephanie Cassin for serving on my supervisory committee and Dr. Stephen Want for sitting on my examining committee. Dr. Cassin, your expertise in the area of eating and weight loss brought great strength and quality to the research. I greatly appreciate your insight, knowledge, and continued presence and commitment to the project. Dr. Want, your enthusiasm for research is contagious.

The recruitment and testing of participants would not have been possible without the help of Kathleen Stewart. Thank you for your commitment, patience, availability, and enthusiasm. I wish you all the best as you begin your Masters degree. Thank you to Dr. Eric Hehman, Philippe Shnaider, and Danielle MacDonald for your statistical consultation. I am truly grateful for your time, willingness to answer questions, and thoughtful recommendations.

Finally, my Masters thesis would not have been possible without the unconditional support, love, and care of my loved ones: Michael, Brigitte, Jared, and Emily Krause, James MacKnight, and Lauren Kennedy. Thank you for your listening ear, genuine interest, cheerleading, and prayers. Through good and bad, you remind me to dream big, to be true to myself, and to pursue wholeheartedly the call that is on my life. 


\section{Table of Contents}

Declaration for Electronic Submission ................................................................................. ii

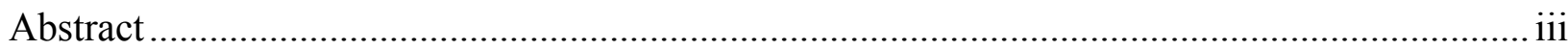

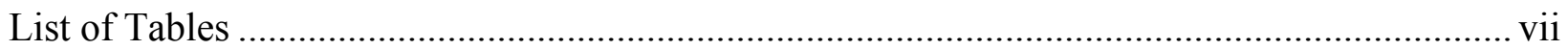

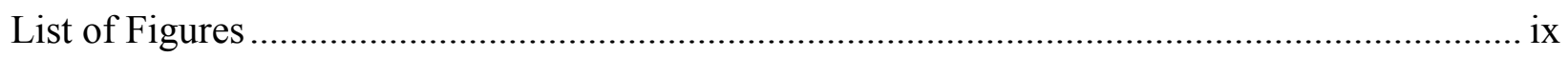

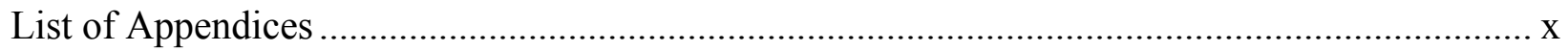

Raising the Bar in Clinical Perfectionism................................................................................. 1

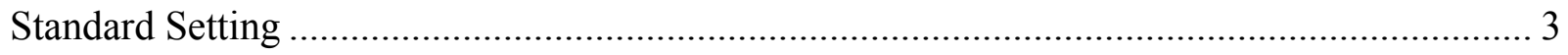

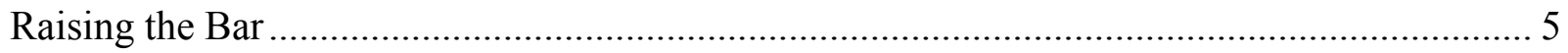

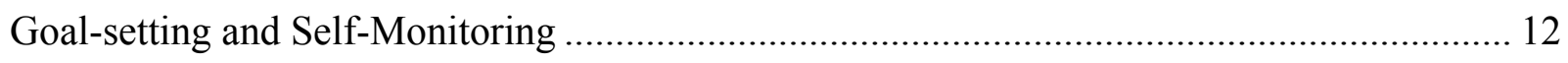

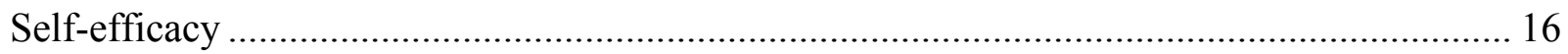

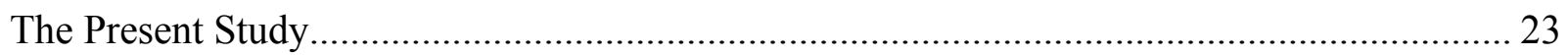

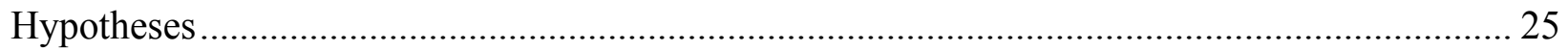

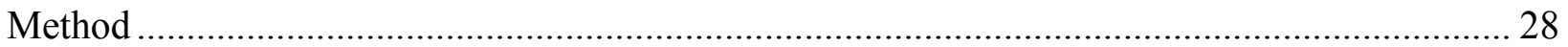

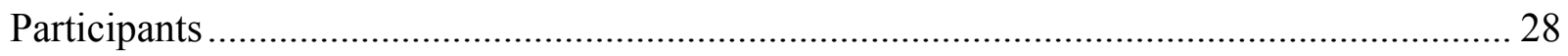

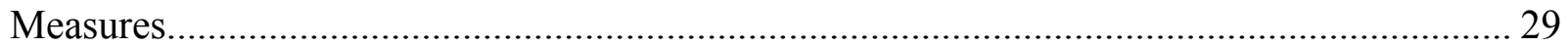

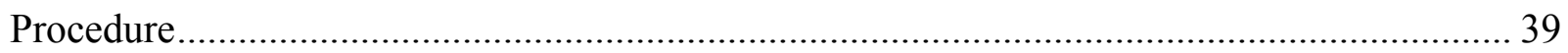

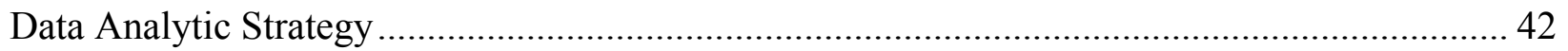

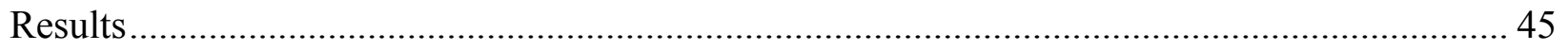

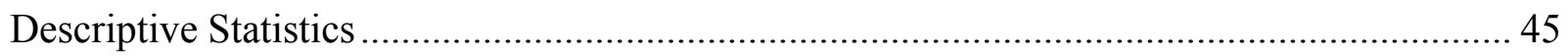

Correlational Analyses: Relationship Between Perfectionism, Self-Efficacy, and Raising the

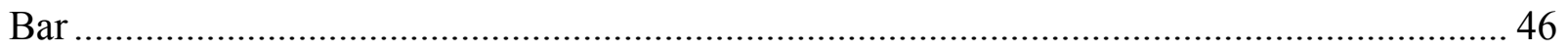

Regression Analyses: Predicting the General Tendency to Raise the Bar ................................ 47 
Regression Analyses: Predicting Weight-Related Raising the Bar.

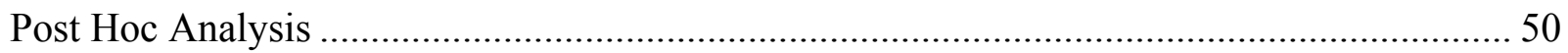

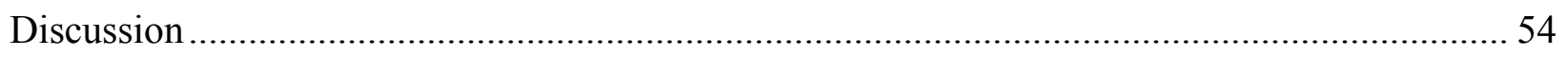

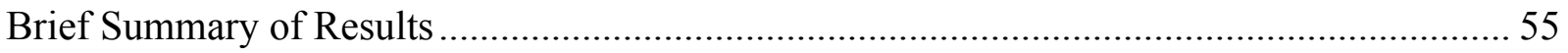

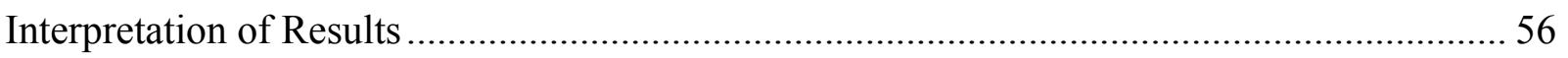

Implications of Findings and Future Recommendations..................................................... 70

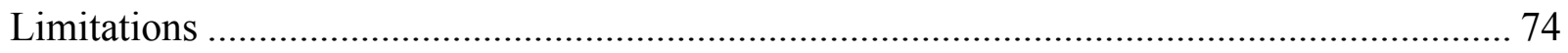

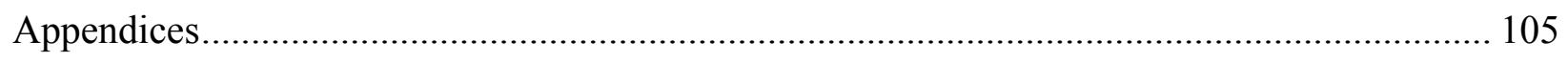

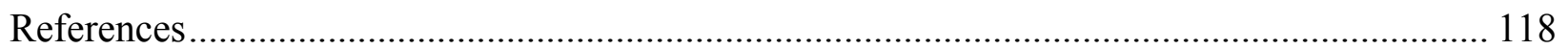




\section{List of Tables}

Table 1: Differences in Measures Between Completers and Noncompleters............................. 80

Table 2: Comparison of Mean Score to Total Possible Score on Primary Variables ................... 81

Table 3: Descriptive Statistics for Weight-Related Goals ...................................................... 82

Table 4: Correlation Matrix for GGSQ, Perfectionism, Self-Efficacy and Psychopathology

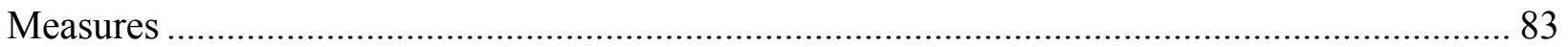

Table 5: Linear Regression for Predicting the General Tendency to Raise the Bar (GGSQ). ..... 84

Table 6: Hierarchical Multiple Regression Analyses Predicting Week 1 Change in Weight-

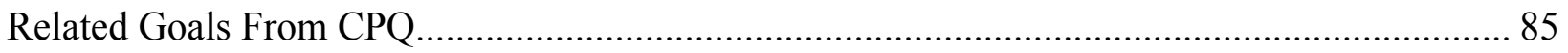

Table 7: Hierarchical Multiple Regression Analyses Predicting Week 1 Change in Weight-

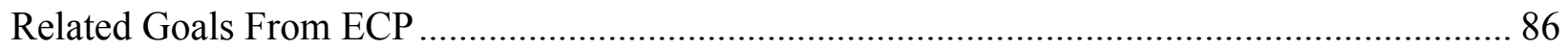

Table 8: Hierarchical Multiple Regression Analyses Predicting Week 1 Change in Weight-

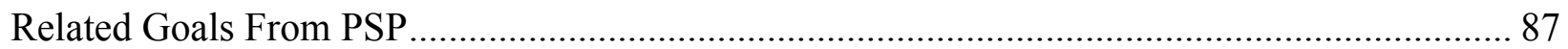

Table 9: Hierarchical Multiple Regression Analyses Predicting Week 2 Change in Weight-

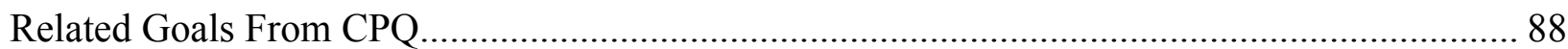

Table 10: Hierarchical Multiple Regression Analyses Predicting Week 2 Change in Weight-

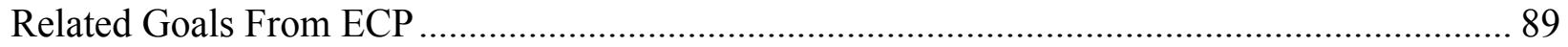

Table 11: Hierarchical Multiple Regression Analyses Predicting Week 2 Change in Weight-

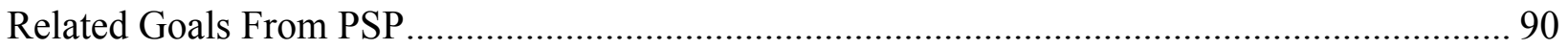

Table 12: Summary of Nonsignificant Simple Slopes for Two-Way Interactions on Weight-

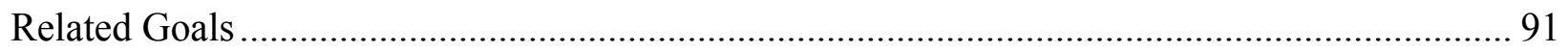


Table 13: Linear Regression Analysis Predicting Total Weight Loss from Perfectionism

Table 14: Description of Reasons for Raising the Bar 93

Table 15: Interrater Agreement for Qualitative Analysis 94

Table 16: Frequency of GGSQ StanD and CPQ Scores in Top Third of GGSQ StanI Participants 95

Table 17: Responses of Participants that Scored in the Top Third of the GGSQ StanI and GGSQ

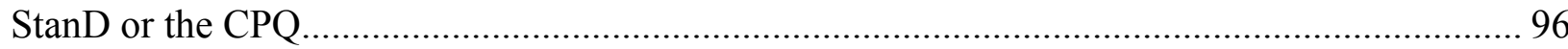

Table 18: Remaining Statements for Self-Improvement with Accompanying GGSQ Factor and CPQ Scores 97 


\section{List of Figures}

Figure 1: Response rate throughout recruitment and testing process.

Figure 2: Simple slopes analysis of ECP by HHG success on Week 1 HHG change.

Figure 3: Simple slopes analysis of CPQ by average HHG self-efficacy on Week 1 HHG change. 100

Figure 4: Simple slopes analysis of ECP by average HHG self-efficacy on Week 1 HHG change. 101

Figure 5: Simple slopes analysis of CPQ by average HHG self-efficacy on Week 2 HHG change. 102

Figure 6: Simple slopes analysis of CPQ by average HHG self-efficacy on Week 2 HHG change. 103

Figure 7: Simple slopes analysis of PSP by average HHG self-efficacy on Week 2 HHG change. 


\section{List of Appendices}

Appendix A Goal-setting Questionnaire .......................................................................... 105

Appendix B General Goal-setting Questionnaire ....................................................... 116

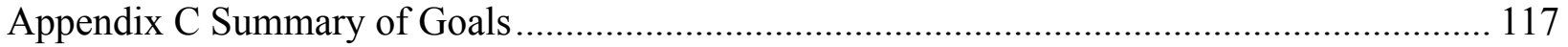




\section{Raising the Bar in Clinical Perfectionism:}

\section{An Evaluation in the Domain of Weight Loss}

Shafran, Cooper, and Fairburn (2002) proposed a cognitive-behavioural model of clinical perfectionism, defining the construct as "the overdependence of self-evaluation on the determined pursuit of personally demanding, self-imposed, standards in at least one highly salient domain, despite adverse consequences" (p. 778). In this model, perfectionistic tendencies are expressed in domains relevant and important to the individual. Self-worth is dependent upon the ability to meet personally challenging and self-set standards in the relevant domain. Failure leads to high levels of negative self-evaluation and self-criticism, and success leads to the reappraisal of previous standards as insufficient. Moreover, clinical perfectionism is understood to be a dysfunctional process, whereby the dependence on self-evaluation results in continual striving toward one's high standards even when this striving results in adverse consequences (e.g., low mood, social withdrawal, fatigue, Shafran et al., 2002).

Although Shafran et al.'s (2002) definition shares features with multidimensional conceptualizations of perfectionism (i.e. standard setting, self-criticism, see Frost, Marten, Lahart, and Rosenblate, 1990; Hewitt and Flett, 1991), the purpose of their model is different from previous models in that it is meant to directly inform clinical treatment and assessment rather than describe an orientation of personality. Specifically, Shafran et al. (2002) criticize multidimensional models for diverting attention away from the theoretical and clinical understanding of perfectionism (i.e., self-evaluation based on high personal standards) by separating it into dimensions that may be related to perfectionism, but perhaps do not reflect the construct itself (i.e., other-oriented perfectionism, parental criticism). This criticism has sparked some debate over the dimensional nature of perfectionism (Dunkley, Blankstein, Masheb, \& 
Grilo, 2006; Hewitt, Flett, Besser, Sherry, \& McGee, 2003; Shafran, Cooper, \& Fairburn, 2003).

Despite the debate over how to best define perfectionism, the definition of clinical perfectionism has proven beneficial in clinical settings. Preliminary research has demonstrated the clinical efficacy of treating clinical perfectionism using Shafran et al.'s (2002) cognitivebehavioural model. Studies include a case study (Shafran, Lee, \& Fairburn, 2004), two case series (Egan \& Hine, 2008; Glover, Brown, Fairburn, \& Shafran, 2007), and a qualitative analysis supporting the core psychopathology and maintaining factors proposed by the model (Riley \& Shafran, 2005). Furthermore, a manualized cognitive-behavioural self-help protocol based on Shafran et al.'s (2002) model has been developed by the authors to treat clinical perfectionism (Shafran, Egan, \& Wade, 2010). To date, one case series (Steele et al., 2013) and three randomized controlled trials (RCT; Egan, van Noort, et al., 2014; Hoiles, Egan, Rees, \& Kane, 2014; Riley, Lee, Cooper, Fairburn, \& Shafran, 2007) have demonstrated the efficacy of this protocol in reducing clinical perfectionism.

Shafran et al.'s (2002) view that perfectionism is dysfunctional is not a novel proposition. According to Shafran et al.'s (2002) model, dysfunctional processes could include the adverse emotional (e.g., low mood), social (e.g., social withdrawal), physical (e.g., fatigue), cognitive (e.g., dichotomous thinking, selective attention on failure), or behavioural (e.g., procrastination) consequences of pursuing excessively high standards. More generally, perfectionism is often referred to as a transdiagnostic process associated with a number of psychological disorders (Egan, Wade, \& Shafran, 2011), including social anxiety disorder (Laurenti, Bruch, \& Hasse, 2008), obsessive-compulsive disorder (Juster et al., 1996; Moretz \& McKay, 2009), panic disorder and agoraphobia (Antony, Purdon, Huta, \& Swinson, 1998; Iketani et al., 2002), eating disorders (Bardone-Cone, Sturm, Lawson, Robinson, \& Smith, 2010), and depression (Enns \& 
Cox, 2005). Perfectionism has also been shown to predict higher levels of comorbidity (Bieling, Summerfeldt, Israeli, \& Antony, 2004). Moreover, it has been suggested that perfectionism may negatively influence treatment outcome by impeding the development of a strong therapeutic alliance (Blatt, Zuroff, Bondi, Sanislow, \& Pilkonis, 1998; Zuroff et al. 2000). Shafran et al. (2002) emphasize the importance of identifying the core dysfunctional characteristics of

clinically relevant perfectionism to inform treatment and assessment, and propose that a strength of the model is its provision of testable hypotheses. However, research on the understanding of key features of the model is still in its early stages. Therefore, the aim of the current study is to test an aspect Shafran et al.'s (2002) model, namely that clinical perfectionists set higher standards following success due to the reappraisal that initial standards were insufficient.

\section{Standard Setting}

Numerous definitions of perfectionism have been proposed over the years. While these definitions differ in various ways, setting high or excessive standards is identified as a core feature of perfectionism in many of these definitions (Egan, Wade, et al., 2014). For example, perfectionism has been described as having "standards [that] are high beyond reach or reason" (Burns, 1980, p. 34) or as "the tendency to believe there is a perfect solution to every problem, that doing everything perfectly (i.e., mistake-free) is not only possible, but also necessary" (Obsessive Compulsive Cognitions Working Group, OCCWG, 1997, p. 678). The setting of high standards is also apparent in multidimensional models, in which perfectionism has been described as "the setting of excessively high standards for performance accompanied by tendencies for overly critical self-evaluations of one's own behaviour" (Frost et al., 1990, p. 450) and as having unrealistic standards for oneself (Self-oriented perfectionism, SOP), for others (Other-oriented perfectionism, OOP), or believing that others have unrealistic standards 
for oneself (Socially prescribed perfectionism, SPP; Hewitt \& Flett, 1991).

A number of studies have examined the role of standard setting in perfectionism. Alden, Bieling, and Wallace (1994) showed that SOP is associated with setting standards that exceed one's perceived ability and with increased importance in achieving those standards in participants with social anxiety disorder and dysphoria. SPP, on the other hand, was associated with repeated self-appraisal but not setting high standards. Bieling and Alden (1997) showed that SPP was associated with the belief that others have high standards for oneself in participants with social anxiety disorder compared to controls. In a study examining the link between perfectionism and affect, Besser, Flett, and Hewitt (2004) manipulated success and failure by providing positive or negative feedback to participants. Findings showed that SOP was associated with significant increases in negative affect, independent of feedback, performance, or task difficulty.

Shafran et al. (2002) also emphasize standard setting as a core feature of clinical perfectionism. However, standard setting on its own is not sufficient to capture the clinical nature of dysfunctional perfectionism, as standard setting can also be seen as a "normal" perfectionistic behaviour (Hamacheck, 1978) that can lead to feelings of satisfaction and bolster self-esteem (Terry-Short, Glynn Owens, Slade, \& Dewey, 1995). Instead, standard setting in clinical perfectionism is maladaptive. Fear and avoidance of failure are thought to motivate perfectionistic striving more than the need for self-improvement. Whether someone is successful in meeting standards is thought to determine self-worth, where unsuccessful attempts to meet those standards result in "self-criticism and negative self-evaluation" (p. 778). Furthermore, there are a number of important elements specific to setting perfectionistic standards within Shafran et al.'s (2002) model. First, standards that the individual sets must be self-imposed. In other words, 
even if someone else initially suggested the goal, it has since been adopted as one's own.

Second, clinical perfectionists have high standards in specific life domains that are relevant and important to them (e.g., eating and weight loss), but not in areas that are unimportant. Third, while other definitions of perfectionism simply mention that standards are excessively high, Shafran et al.'s (2002) definition specifies that standards must be personally demanding, emphasizing the subjective nature of the difficulty and effort exerted. Finally, because individuals with clinical perfectionism are persistently striving to do better, standards are continually being set and reset.

\section{Raising the Bar}

The resetting of standards has been referred to as raising the bar (Shafran et al., 2002), and was the focus of the present study. According to the model, when clinical perfectionists successfully meet their demanding and stringent standards, they reappraise those standards as not being sufficiently challenging. While the experience of success may be temporarily reinforcing, a person with clinical perfectionism will not be satisfied for long, and will instead raise the bar, setting him or herself up for failure (Shafran et al., 2002). Consistent with this component of the model, a qualitative analysis by Riley and Shafran (2005) demonstrated that positive emotions following success in those with clinical perfectionism were more short-lived than in those without clinical perfectionism.

Some preliminary research has examined raising the bar more directly. Kobori, Hayakawa, and Tanno (2009) examined raising the bar using the Stroop task. Participants were informed that, on average, $15 \%$ of the trials on a Stroop task are answered incorrectly. Participants were asked to attempt to perform at least as well as average (i.e., less than or equal to $15 \%$ answered incorrectly). Success was manipulated by informing participants that they had 
performed above average, regardless of actual performance. Participants then had the choice between two goals for a second Stroop task: answer less than $15 \%$ or less than $10 \%$ incorrectly. They were also told that if they did not achieve their goal, their honorarium would be decreased by half. Results revealed that participants high in SOP were more likely to raise the bar (i.e., choose 10\%) for the second Stroop task. Although these results are muddled by threatening to reduce the incentive, it could be suggested that an aversive consequence of failure actually bolsters the findings. One might expect that the consequence of less money would prevent participants from choosing the more challenging goal. However, in spite of this consequence, perfectionism still predicted raising the bar.

Lo and Abbott (2013a) explored the relationship between standard setting and adaptive perfectionists, maladaptive perfectionists, and nonperfectionists. These categories were determined using cutoff scores developed by Rice and Ashby (2007). Those scoring high in the high standards subscale (i.e., setting elevated standards for the self) on the Almost Perfect Scale Revised (APS-R; Slaney, Rice, Mobley, Trippi, \& Ashby, 2001) were classified as perfectionists. Of this group, those who scored high vs. low on the discrepancy subscale (i.e., the perception of continuously failing to achieve one's elevated standards) of the APS-R were classified as maladaptive and adaptive perfectionists respectively (Lo \& Abbott, 2013a). Using a procedure similar to Kobori et al. (2009), participants were asked after completing an anagram task whether they wanted to complete a second anagram task of similar or increased difficulty (i.e. raising the bar). Half of the participants were placed in a low expectations group, where they were told that 3 out of the 15 anagrams should be solved by the end of the task, and the other half of participants were placed in a high expectations group, where they were told that 12 out of the 15 anagrams should be solved by the end of the task. When in the low expectations group, adaptive 
perfectionists and nonperfectionists were significantly more likely to chose a second anagram of increased difficulty than one of similar difficulty (i.e., $67 \%$ and $63 \%$ chose a more difficult anagram, respectively). Likewise, when in the high expectations group, adaptive perfectionists and nonperfectionists were significantly more likely to choose a second anagram of similar difficulty than one of increased difficulty (i.e., $94 \%$ and $80 \%$ chose an similarly difficult anagram respectively). Although results were not significant for maladaptive perfectionists, this group showed a similar pattern to both adaptive perfectionists and nonperfectionists, with $61 \%$ of maladaptive perfectionists choosing a more difficult anagram when in the low expectations group, and $67 \%$ choosing an equally difficult anagram when in the high expectations group. This suggests that raising the bar occurs for an achievable goal (i.e., solving more than 3 of 12 anagrams), but not when the goal is already sufficiently demanding, regardless of type or level of perfectionism (i.e., solving more than 12 of 15 anagrams, Lo \& Abbott, 2013a).

Stoeber, Hutchfield, and Wood (2008) examined the impact of success or failure feedback on perfectionistic striving, aspiration level, self-efficacy, and self-criticism. Participants were asked to complete one of seven tests of seemingly increasing difficulty level, whereas in actuality each test was the same. Following completion of the first test, participants were provided with false success or failure feedback regarding their performance, and were then asked to select the difficulty level of a second test. The choice of a second test was viewed as a measure of aspiration level. Therefore, although these researchers did not base their study on Shafran et al.'s (2002) conceptualization of clinical perfectionism, choosing a second test was conceptually similar to raising the bar. Results revealed that participants high in perfectionism, as measured by the Striving for Perfection Scale (SPS; Stoeber, Otto, Perscheck, Becker, \& Stoll, 2007), showed increases in aspiration level (i.e., raising the bar) following success feedback. In 
contrast, participants high in self-criticism showed decreases in aspiration level regardless of success or failure.

Egan, Dick, and Allen (2012) examined raising the bar after both success and failure. This study improved on previous research by predicating performance on actual success or failure rather than manipulating feedback. Participants completed three sets of a nonverbal reasoning test. For the first set of questions, participants set a goal for the number of questions they wanted to answer correctly. After receiving feedback about their performance, participants were asked to set a goal for the second set of questions, and so forth. Results revealed that clinical perfectionism, as measured by the Clinical Perfectionism Questionnaire (CPQ; Fairburn, Cooper, \& Shafran, 2003a), was associated with setting higher standards for the first set of questions, such that participants higher in clinical perfectionism set higher goals. However, this association was not present for the second or third set of questions, indicating that clinical perfectionists did not raise the bar after success or failure.

Two major limitations prevent an integrated interpretation of these studies. First, perfectionism is defined and measured in different ways across studies. Kobori et al. (2009) used the Japanese version (Otani \& Sakurai, 1995) of the Hewitt and Flett Multidimensional Perfectionism Scale (HMPS, Hewitt \& Flett, 1991, see Measures section). Lo and Abbott (2013a) assessed adaptive and maladaptive perfectionism as measured by the APS-R (Slaney et al., 2001). Stoeber et al. (2008) refer to perfectionistic striving as measured by the SPS (Stoeber et al., 2007). Of all the available studies examining the model-specific construct of raising the bar, only Egan et al. (2012) used the CPQ (Fairburn et al., 2003a), measuring perfectionism as conceptualized by Shafran et al. (2002). At the very least, this measure should be included alongside other commonly used measures of perfectionism. Therefore, given the various 
methodological choices employed, it is not surprising to find such diverse and sometimes contradictory results across studies.

Second, and more importantly, these studies examine raising the bar in an artificial laboratory setting using tasks that are not necessarily personally relevant to individuals with clinical perfectionism. Shafran et al.'s (2002) model suggests that clinical perfectionism presents in life domains that are important and relevant to the individual, and that standards must be selfimposed and personally demanding. The underlying assumption in the previously reviewed studies is that completing an aptitude test or cognitive task in a laboratory is synonymous with standard setting in a relevant and important life domain (e.g., weight, work, school). However, laboratory tasks used to examine raising the bar have not been self-imposed, but rather are chosen by the experimenter (e.g., Stroop or anagram task). Moreover, whether a task is personally demanding is dependent on the individual. What is difficult for one participant may be easy for another. According to Shafran et al. (2002), raising the bar occurs after perceived success. With the exception of Egan et al. (2012), past studies have manipulated success and failure feedback. Egan et al. (2012) suggested that false feedback may reduce the face validity of the task such that participants may be able to recognize the disconnect between actual performance and the feedback received. What is truly important in the cognitive-behavioural model of clinical perfectionism is whether the individual perceives that he or she was successful, rather than whether he or she received positive or negative performance feedback.

One study successfully examined standard setting in a salient life domain. Extending findings from a previous study (Brown et al., 1999), Bieling, Israeli, Smith, and Antony (2003) asked a sample of university students to report on their behaviours, standards, attributions, and perfectionism at two points in time: (1) 1 week before a midterm exam and (2) after receiving 
their midterm results. Specifically, Bieling et al. (2003) collected information regarding exam standards, the discrepancy between standards and performance, positive and negative affect, exam attributions, and standards for future exams. Perfectionism was measured using the HMPS, Frost et al. Multidimensional Perfectionism Scale (FMPS, Frost et al., 1990; see Measures section), as well as composite perfectionism scores created using subscales of the HMPS and FMPS (Frost, Heimberg, Holt, Mattia, \& Neubauer, 1993; Bieling, Israeli, \& Antony, 2004). These composite scores were defined as (1) positive achievement striving, conceptualized as an adaptive form of perfectionism associated with positive affect (Frost et al., 1993), and (2) maladaptive evaluative concerns perfectionism, conceptualized as a maladaptive form of perfectionism associated with negative affect, depression, and anxiety (Frost et al., 1993; Bieling et al., 2004). Results revealed that higher perfectionism scores on the FMPS, positive achievement striving, and maladaptive evaluative concerns perfectionism were associated with setting higher midterm standards, feeling more negative affect about the midterm, not achieving one's expected results, planning to study more for future exams, and setting higher standards for future exams. Interestingly, when looking at the positive achievement striving composite score and the maladaptive evaluative concerns composite perfectionism scores separately, positive achievement striving predicted setting higher standards for future exams, whereas maladaptive evaluative concerns did not.

In their seminal article on clinical perfectionism, Shafran et al.'s (2002) cognitivebehavioural model was evaluated in the context of eating disorders. They proposed that perfectionism and eating disorders are not simply co-occurring issues, but rather that eating disorders are "the expression of perfectionism in the domain of eating, shape, or weight and their control" (p. 783). In fact, Fairburn, Cooper, and Shafran (2003b) describe clinical perfectionism 
as one of the four maintaining mechanisms of eating pathology. Two experimental studies have demonstrated the relevance of standard setting in the context of eating and weight loss. Using a nonclinical female sample, Shafran et al. (2006) experimentally manipulated personal standards, a core element of clinical perfectionism, to explore their impact on eating patterns, behaviours, and attitudes. Participants signed a contract to do everything in their lives to either the highest or the lowest possible standard for the following 24 hours. Results revealed that participants in the high personal standards condition consumed fewer high calorie foods, invested more effort to restrict overall intake of food, and felt more regret after eating than participants in the low personal standards condition. Replicating Shafran et al.'s (2006) procedure, Boone, Soenens, Vansteenkiste, and Braet (2012) found that participants attempting to uphold high personal standards for 24 hours reported more restrained eating and binge eating than participants asked to maintain low standards. While these studies were experimental, and therefore standards were not self-imposed, it follows that eating and weight loss is a relevant and important life domain in which to examine standard setting and raising the bar.

Therefore, the purpose of the present study was to examine the phenomenon of raising the bar as hypothesized by Shafran et al.'s (2002) model of clinical perfectionism in a sample of individuals who are overweight or obese and wish to lose weight. Individuals were asked to set and reset a number of personal weight-related goals at three time points while using a mobile application (“app") with which they self-monitored food intake and exercise. The terms "standards" and "goals" were synonymous for the purpose of the current study. This design was chosen because it (1) examined raising the bar in a real world setting (i.e., self-monitoring of actual food consumption and energy expenditure) and (2) involved a self-imposed and personally demanding task (i.e., desire to lose weight and selecting personal weight-related goals). Goal- 
setting and self-monitoring are considered to be the cornerstone of behavioural weight loss interventions (Levy, Finch, Crowell, Talley \& Jeffery, 2007). An explanation of goal-setting and self-monitoring, as well as a theoretical and empirical review of their relevance to weight loss is provided below. Additionally, self-efficacy has been shown to be associated with performance (Bandura, 1977), goal-setting (Locke \& Latham, 1990), and perfectionism (e.g., Martin, Flett, Hewitt, Krames, \& Szanto, 1996). Although Shafran et al. (2002) do not address self-efficacy in their model, one's beliefs regarding the ability to accomplish a goal must be taken into consideration when examining raising the bar. Therefore, a review of self-efficacy's relationship to performance, goal-setting, and perfectionism is also provided.

\section{Goal-setting and Self-Monitoring}

Goal-setting and self-monitoring are understood to be effective weight loss strategies, particularly when used together (Levy et al., 2007). Specifically, guidelines for the treatment of obesity have identified behaviour modification strategies such as self-monitoring and setting realistic goals as integral features of planned weight loss interventions, alongside the reduction of energy intake and increase of energy expenditure (American Gastroenterological Association, 2002; Jensen et al., 2014). In a qualitative study, McKee, Ntoumanis, and Smith (2013) found that individuals who successfully maintained weight loss 1 year after a weight loss intervention reported engaging in consistent self-monitoring and realistic goal-setting. Additionally, a metaregression revealed that weight loss interventions are most effective when they include selfmonitoring in combination with at least one other self-regulatory strategy, one of which is goalsetting (Michie, Abraham, Whittington, and McAteer, \& Gupta, 2009)

Goal-setting. The overarching finding in research evaluating goal-setting is that specific, challenging goals produce better performance and outcomes than easy, "do-your-best" goals. For 
example, a goal where one intends to exercise more is vague and nonspecific, and not likely to achieve positive results. Conversely, a goal where one intends to take a brisk 20-minute daily walk is manageable, measureable through a food and exercise diary, and focused on a specific behaviour (Levy et al., 2007). This finding that specific goals are better than "do-your-best" goals is robust and has been demonstrated in over 100 separate tasks, including more than 40000 participants in a number of different research settings and countries around the world (Locke \& Latham, 2002). Branching out of organizational psychology, the utility of goal-setting as a behavioural change strategy has been examined in a number of health domains including, but not limited to, diabetes (De Walt et al., 2009), smoking (Borrelli \& Mermelstein, 1994), coronary heart disease (Fernandez, Rajaratnam, Evans, \& Speizer, 2012), alcohol consumption (Cho, 2007), and related to the current study, exercise and dietary behaviour (Kyllo \& Landers, 1995; Pearson, 2012). In a meta-analysis, Kyllo and Landers (1995) revealed that setting goals improved sport and exercise performance by 0.34 of a standard deviation. In a systematic review evaluating the utility of goal-setting as a strategy for dietary and physical activity behavioural change, Pearson (2012) found that combining specific short-term goals with performance feedback and self-monitoring resulted in a number of positive outcomes including reductions in weight, Body Mass Index (BMI; an individual's body mass as measured by weight in kilograms divided by the square of height in meters), food consumption, and increases in exercise and intention to engage in healthy behaviours.

Performance is improved when challenging, specific goals are set, because they act to direct the individual's attention towards goal-consistent behaviour, and increase effort and persistence. In other words, individuals will be more focused, expend greater effort, and work longer and harder to achieve a challenging, specific goal than they will to achieve a vague, 
nonspecific goal (Locke \& Latham, 1990). For example, De Vet, Nelissen, Zeelenberg, and De Ridder (2012) found that setting higher weight loss goals predicted increased self-reported effort to achieve those goals. Therefore, setting goals that are specific and focused on the behaviour are recommended to achieve success.

Self-Monitoring. Self-monitoring involves documenting one's food and drink intake, exercise, and weight loss. Its purpose is to increase the overall awareness of positive and negative eating patterns, and environmental or psychological stimuli that contribute to episodes of overeating. It serves both as an assessment and treatment planning tool as well as a progress report, and allows the individual to set specific goals that target the antecedents and consequences of overeating episodes. For example, if going to the grocery store on an empty stomach (antecedent) triggers an episode of overeating, then a goal could be to grocery shop on a full stomach. Likewise, following an episode of overeating (consequence), goals can be tailored to avoid relapse (Levy et al., 2007).

In a systematic review of dietary, exercise, and weighing self-monitoring studies, Burke, Wang, and Sevick (2011) identified a consistent positive relationship between self-monitoring and weight loss. Important to this relationship is the consistency with which one monitors food intake when attempting to lose weight. In a number of early studies that examined the impact of self-monitoring on weight loss, participants who kept more consistent food diaries lost more weight than participants who kept less consistent food diaries (Baker \& Kirschenbaum, 1993; Baker \& Kirschenbaum, 1998; Boutelle \& Kirschenbaum, 1998; Boutelle, Kirschenbaum, Baker, $\&$ Mitchell, 1999). The frequency of self-monitoring has also been associated with greater outcomes such as weight loss percentage (Burke et al., 2008). 
Research suggests that the method of self-monitoring in weight loss programs should be tailored to meet individual preferences (Shay, Seibert, Watts, Sbrocco, \& Pagliara, 2009; Yon, Johnson, Harvey-Berino, Gold, \& Howard. 2007). For example, Shay et al. (2009) found that adherence to self-monitoring was higher when participants were able to choose their preferred method of tracking (paper dairy vs. personal digital assistant [PDA], vs. website), and that different methods did not result in different anthropometric outcomes.

Mobile Self-Monitoring App. The current study used a smartphone app to self-monitor food consumption and energy expenditure. Smartphone apps for promoting weight-related behavioural change are a more recent and attractive alternative to pen and paper or PDA methods of self-monitoring, given their constant accessibility, ability to tailor the program to conform to the needs of the client, and provision of automated feedback (Middelweerd, Mollee, van der Wal, Brug, \& te Velde, 2014). A number of studies have demonstrated the utility of specific weightrelated self-monitoring apps (Lee, Chae, Kim, Ho, \& Choi, 2010; Mattila, Lappalainen, Pärkkä, Salminen and Korhonen, 2010; Tsai et al., 2007). One study found that self-monitoring and goalsetting on a smartphone combined with texted feedback resulted in significantly more weight loss and a larger waist reduction than a no-treatment control group at 1-year follow-up (Haapala, Barengo, Biggs, Surakka, \& Manninen, 2009). In another study, Carter, Burley, Nukjaer, and Cade (2013) conducted an RCT to compare a smartphone weight loss self-monitoring app to both a website version of the app and a pen and paper method. Like Happala et al. (2009), this smartphone app incorporated self-monitoring, goal-setting, and text message feedback. Attrition was significantly higher, and adherence to self-monitoring significantly lower in both the website and pen and paper diary groups compared to the smartphone group. As this was a pilot trial, the RCT did not have sufficient power to detect differences in anthropomorphic measures such as 
weight change and BMI across groups. However, using intent-to-treat data, the smartphone group was found to lose significantly more weight at 6 months than the other two groups. Moreover, weight loss by the smartphone group was comparable to other 6-month diet interventions (Carter et al., 2013). Likewise, Wharton, Johnston, Cunningham, and Sterner (2014) found that participants using a smartphone app for weight loss were significantly more consistent in self-monitoring food intake than participants using a pen and paper diary. However, while adherence was greater for the smartphone app group, all groups experienced a significant decrease in weight and BMI. Interestingly, self-monitoring consistency did not differ across the smartphone app group and a group that self-monitored using an electronic memo pad on their iPhone. In summary, the literature demonstrates not only that self-monitoring and goal-setting are effective strategies for weight loss, but that these methods can be administered effectively via smartphone app.

\section{Self-efficacy}

In his social-cognitive theory, Bandura (1977) defined self-efficacy as an individual's belief that he or she can successfully execute a behaviour necessary to produce a desired outcome. Self-efficacy is strengthened in four ways: (1) by directly experiencing success (mastery), (2) by seeing others successfully complete a task (modeling), (3) by being verbally encouraged or exhorted (persuasion), or (4) by not experiencing states of aversive arousal when completing a task (physiological feedback). According to Bandura's (1977) model, an individual's level of self-efficacy determines the amount of effort he or she will contribute to a task, and the time he or she will persist at a task when faced with problems or obstacles before abandoning it. Empirically, Bandura (1977) found a positive relationship between self-efficacy and performance, in that the better one expects to perform, the more successful the outcome. The 
positive relationship between self-efficacy and improved performance is empirically supported (see Locke \& Latham, 1990 for review).

Self-efficacy and goal-setting. As mentioned previously, goal-setting theory notes that the effort, persistence, and ultimately the performance of an individual are improved by setting challenging, specific goals (Locke \& Latham, 1990). It follows that the outcome of a certain executed behaviour, such as engaging in weight loss strategies like goal-setting and selfmonitoring, is influenced not only by the strategies themselves, but also by psychosocial processes such as self-efficacy. Indeed, a 35-year review of the goal-setting literature shows that individuals with higher self-efficacy set higher personal goals, have greater commitment to goals assigned to them, use better strategies to successfully complete the goals, and have more positive responses to negative feedback than do individuals with low self-efficacy (Locke \& Latham, 2002).

Self-efficacy has been examined in the context of health related goal-setting. Higher selfefficacy has been shown to significantly predict positive short- and long-term outcomes in a number of different health domains, including smoking cessation, use of contraception, alcohol consumption, exercise behaviours, and weight loss or maintenance (Strecher, DeVellis, Becker, \& Rosenstock, 1986). In a sample of adult overweight females, Dennis and Goldberg (1996) identified two types of weight-control self-efficacy beliefs using factor analysis of Q sorts. The first type, labeled assured women, were characterized by high self-efficacy in that they exhibited an independent and persistent attitude toward weight control, faced problems head on, and were unlikely to give up easily. They were also goal-directed and confident in their abilities to lose weight. The second type, labeled disbelieving women, had low self-esteem and displayed the opposite characteristics of the assured women. Results from the study revealed that assured 
women lost significantly more weight than disbelieving women. Moreover, women who transitioned from disbelieving to assured over the course of the intervention lost twice as much weight than disbelieving women. Assuredness was also associated with higher self-esteem, better mood, and more positive eating patterns. These results show how the outcome of the same weight loss intervention can be dependent upon an individual's level of self-efficacy.

In a commentary on social-cognitive theory and health promotion, Bandura (1998) explained how self-efficacy and self-regulatory behaviours such as self-monitoring or goalsetting are interconnected. Specifically, self-efficacy influences the perceived cause of success and failure through self-monitoring and determines the difficulty of, and commitment to, chosen goals. Therefore, given that performance, goal-setting, and feedback are integral parts of many weight loss interventions, and that self-efficacy influences these processes, it makes sense that measures of self-efficacy would be commonly incorporated into studies evaluating the efficacy of overall weight loss interventions or specific behavioural strategies such as goal-setting or selfmonitoring (e.g., Burke et al., 2009; De Vet, et al., 2012; Haapala et al., 2009; Napolitano, Hayes, Bennett, Ives, \& Foster, 2013; Shay et al., 2009). Without accounting for an individual's perceived ability to successfully engage in a weight loss strategy such as self-monitoring or goalsetting, it becomes difficult to piece together whether outcomes are due to inappropriate goalsetting, ineffective interventions, or simply low self-efficacy. In other words, measuring selfefficacy provides a clearer picture of the mechanisms involved in the outcomes of a weight loss intervention.

A consistent relationship exists between high levels of self-efficacy and success in weight loss interventions (Byrne, 2002; Elfhag \& Rossner, 2005; Gormally, Rardin, \& Black, 1980; Jeffery et al., 1984). Moreover, higher self-efficacy significantly predicts future weight loss 
(Haapala et al., 2009; Rodin, Elias, Silberstein, \& Wagner, 1988; Teixeira et al., 2004), BMI reduction (Stotland \& Zuroff, 1991), and engagement in exercise and dieting behaviours such as eliminating sweets between meals, removing fatty or starchy foods from one's diet, or decreasing overall food intake (Bagozzi \& Edwards, 2000; Conn, 1998). Self-efficacy has also been shown to mediate the relationship between the use of behavioural weight loss strategies and positive weight-related or dietary health outcomes. Kitsantas (2000) found that higher self-efficacy to implement behavioural weight loss strategies, such as self-monitoring, goal-setting, seeking information or help from others, and environmental structuring, is associated with increased weight loss. Participants of healthy weight, or those who had maintained weight loss used more strategies and had higher self-efficacy about using strategies than a group of overweight participants. In another study attempting to increase dietary fiber consumption, Schnoll and Zimmerman (2001) had college nutrition students participate in a goal-setting and selfmonitoring study. Results revealed that students who set goals performed better than students who did not set goals. Students who set goals also reported higher dietary fiber self-efficacy. Importantly, the impact of self-monitoring and goal-setting on fiber consumption was mediated by dietary fiber self-efficacy. That is, strategies such as self-monitoring and goal-setting acted to increase self-efficacy, and it was this combination that resulted in positive outcomes.

Self-efficacy and perfectionism. Given what is known about self-efficacy's relationship with performance, and its mediational role between goal-setting and behavioural change outcomes, self-efficacy may be an important construct to consider when examining perfectionism. That is, perfectionists are known to strive to achieve excessively high standards and goals. Conceptually, a perfectionist's perceived belief in his or her ability to achieve those goals may have an impact on which goals are set and on the outcome of the task (Lo \& Abbott, 
2013b). Burns (1980) posited that perfectionism undermines self-efficacy. The higher the standard, the less likely it is that one will believe a successful outcome is possible. Therefore, according to Burns (1980), self-efficacy is expected to be low in perfectionists because they hold excessively high standards. On the contrary, Bandura (1986) suggested that the high expectations of the perfectionist may improve performance (Lo \& Abbott, 2013b). Successful performance of increasingly challenging goals is understood to foster self-efficacy. Therefore, if the high standards of the perfectionist are successfully achieved, this will bolster self-efficacy. Much of the research examining perfectionism and self-efficacy is correlational. Some findings reveal negative associations in line with Burns' (1980) conceptualization (e.g., Kralj, 1989), while others reveal positive associations, more in line with Bandura's (1986) conceptualization. As perfectionism is often considered a multidimensional construct (e.g., Frost et al., 1990; Hewitt \& Flett, 1991), these positive and negative associations differ depending on the dimension of perfectionism under consideration. For example, Martin et al. (1996) found that while SOP and OOP were positively correlated with a measure of general self-efficacy, a negative correlation was found for SPP and self-efficacy. The authors suggested that items indicating parental criticism in SPP could explain the negative correlation with self-efficacy in that unsupportive parents may undermine a high level of self-efficacy. Mills and Blankstein (2000) found similar patterns. Hart, Gilner, Handal, and Gfeller (1998) found the opposite pattern, in which SOP and OOP were negatively correlated with self-efficacy, and SPP was positively correlated with selfefficacy. Here, the authors suggested that because SPP originates outside the individual, he or she can dismiss unrealistic standards and preserve self-efficacy, whereas those individuals who are high in SOP and SOP are unable to recognize the excessive nature of their standards, therefore damaging self-efficacy. 
Self-efficacy assessments have been included as adjunct measures in a select number of the perfectionism studies focusing on standard setting. When controlling for self-criticism, Stoeber et al. (2008) found that perfectionistic striving, as measured by the SPS (Stoeber et al., 2007) was positively correlated with self-efficacy. Additionally, a number of studies have examined the relationship between adaptive and maladaptive perfectionism, as measured by the APS-R, and self-efficacy (Ganske \& Ashby, 2007; Lo \& Abbott, 2013a; LoCicero \& Ashby, 2000; Zhang \& Cai, 2012; Zhang, Xie, \& Cai, 2013). The consistent finding from these studies is that adaptive perfectionism is associated with higher levels of self-efficacy than maladaptive perfectionism and nonperfectionism. Lower levels of self-efficacy in maladaptive perfectionism are understandable given that maladaptive perfectionism in the APS-R is measured by items that capture distress caused by a dissonance between goals and outcome (e.g., My performance rarely measures up to my standards). In other words, the discrepancy scale measures the failure of individuals to live up to their excessively high standards (Egan, Wade, Shafran, \& Antony, 2014). In a study by Alden et al. (1994), individuals high in SOP set goals or standards that exceeded their level of social self-efficacy.

Gosselin (2003) experimentally explored the impact of feedback on self-efficacy in perfectionism. Participants were split into groups of low, moderate, or high perfectionism depending on scores on the Brief Perfectionism Scale (BPS, Gosselin, Boone, Sinek, \& Tangney, 2002). After completing a word task, participants were provided with either negative or neutral feedback on their performance. Participants low in perfectionism demonstrated a significant pre to posttest decrease in self-efficacy following negative feedback, whereas individuals high in perfectionism maintained the same level of self-efficacy pre to posttest following negative feedback. The authors interpreted this finding to mean that individuals high in perfectionism 
attend more closely to personal feedback than to external feedback, avoiding potential blows to self-efficacy.

No known studies have yet explored the relationship between self-efficacy and clinical perfectionism, as defined by Shafran et al. (2002). Moreover, although Shafran et al.'s (2002) cognitive-behavioural model of clinical perfectionism discusses goal-setting and raising the bar, it does not address the role of self-efficacy. Whether an individual with clinical perfectionism raises the bar, or sets a more challenging goal may be dependent upon his or her level of selfefficacy. As dysfunctional self-evaluation, self-criticism, and fear of failure are key elements of the model, it is possible that clinical perfectionism may hinder high levels of self-efficacy. Conceptually, it follows that clinical perfectionism could be negatively related with self-efficacy. Given the interconnected relationship between goal-setting and self-efficacy, and the fact that self-efficacy has yet to be examined in the context of clinical perfectionism, it was explored in the present study.

Self-efficacy is a domain specific construct rather than a global and generalized trait, in that an individual's perceived ability to execute a task will change from context to context. Therefore, measures of self-efficacy are often tailored to the specific task or behaviour in question (Bandura, 2006). In the present study, self-efficacy was examined in two ways. In line with Bandura's (2006) suggestion, the first method of measuring self-efficacy in the current study was to ask participants to rate the extent to which they believe they were able to achieve a number of weight-related goals they had selected. The second method was to administer the Eating Self-Efficacy Scale (ESES; Glynn \& Ruderman, 1986), a self-report scale developed to assess the perceived ability of dieting or overweight individuals to control overeating. Administering the ESES to participants in a 10-session behavioural weight loss program, Glynn 
and Ruderman (1986) found a significant increase in eating self-efficacy over the course of the intervention, and that these increases were significantly correlated with weight loss. In another study, Peters (1996) found that eating self-efficacy was higher in individuals who had maintained weight loss for longer than 1.5 years compared to individuals who had regained the weight, or control individuals who did not have a history of attempting to lose weight. Improvements in eating self-efficacy over the course of other dietary interventions have been observed (Irwin, 1998; McCann et al., 1995; Prezioso, 1995). Studies have yet to examine perfectionism and eating self-efficacy in the context of goal-setting and weight loss interventions. Using a female university sample, Bardone-Cone, Brownstone, Higgins, Harney, and Fitzsimmons-Craft (2012) used a subset of items from the ESES examining difficulty controlling overeating when experiencing negative affect to explore the relationship between perfectionism and self-efficacy. SOP and SPP were associated with lower levels of eating self-efficacy. However, only SPP interacted with school-related stress to predict low eating self-efficacy when experiencing negative affect.

\section{The Present Study}

According to Shafran et al. (2002), clinical perfectionism is characterized by the negative self-evaluation of the outcome of personally challenging, individually chosen standards in salient life domains. With the exception of Bieling et al.'s (2003) study, which examined perfectionism in a real world academic setting, previous research exploring standard setting and raising the bar has taken place in laboratory settings with experimenter-imposed tasks that do not necessarily align with domains that are important and relevant to the individual. Therefore, the purpose of the present study was to improve upon limitations in previous studies by examining the phenomenon of raising the bar in a real world setting. Given the conceptual link between clinical 
perfectionism and eating disorders (Shafran et al., 2002), raising the bar was explored in the area of eating and weight loss. To ensure that the domain was self-imposed, a group of individuals who were overweight or obese and desired to lose weight completed a 2-week self-monitoring food and exercise diary using a smartphone app. During this time, they set and reset a number of weight-related goals (e.g., weight loss, calorie consumption, exercise, and healthy habit goals). This served as a measure of raising the bar. In addition, participants were asked about their general tendency to raise the bar after success. As raising the bar is hypothesized in the model to occur after success, weight-related perceived success was evaluated. Furthermore, the influence of self-efficacy was also examined given its relationship with overall performance, goal-setting, and perfectionism. 


\section{Hypotheses}

\section{Correlational Hypotheses: Relationship Between Perfectionism, Self-Efficacy, and Raising}

\section{the Bar}

1. It was predicted that there would be significant associations between perfectionism, selfefficacy, and the general tendency to raise the bar. Specifically,

a. A positive correlation was expected between clinical perfectionism, as measured by the $\mathrm{CPQ}$, and the general tendency to raise the bar, as measured by the General Goal-setting Questionnaire (GGSQ).

b. A positive correlation was expected between general eating self-efficacy, as measured by the ESES, and the general tendency to raise the bar.

c. A negative correlation was expected between general eating self-efficacy and both clinical perfectionism and a maladaptive composite perfectionism score (Evaluative concerns perfectionism, ECP, Dunkley, Blankstein, Halsall, Williams, \& Winkworth, 2000; Stoeber \& Otto, 2006)

d. A positive correlation was expected between general eating self-efficacy and an adaptive composite perfectionism score (Personal standards perfectionism, PSP, Dunkley et al., 2000; Stoeber \& Otto, 2006).

\section{Regression Hypotheses: Predicting the General Tendency to Raise the Bar}

2. It was predicted that perfectionism (i.e., CPQ, ECP) and general eating self-efficacy, would significantly predict the overall tendency to raise the bar, such that higher scores on these measures would result in a greater tendency to raise the bar.

3. Bieling et al. (2003) found that adaptive perfectionism, but not maladaptive perfectionism, as measured by Frost et al. (1993), significantly predicted setting higher standards for an 
upcoming exam. These measurements have since been refined by ECP and PSP (Dunkley et al., 2000). Shafran et al. (2002) did not conceptualize raising the bar as an adaptive behaviour; however, it could be that those high in adaptive perfectionism may raise the bar in a realistic way as a goal-setting tactic or personal challenge. Therefore, the study investigated whether PSP significantly predicted a greater tendency to raise the bar.

\section{Regression Hypotheses: Predicting Weight-Related Raising the Bar}

4. It was predicted that clinical perfectionism would significantly predict raising the bar in all weight related goals (i.e., weight loss, calorie consumption, exercise, and healthy habit goals), such that higher scores in clinical perfectionism would predict a significant increase in goals on Week 1 (Day 1 to Day 8) and Week 2 (Day 8 to Day 15).

5. Shafran et al. (2002) conceptualize clinical perfectionism as dysfunctional, linking it to maladaptive traits such as fear of failure and self-criticism. Moreover, the model hypothesizes that the pursuit of excessively high standards is continued despite adverse consequences. This is related to the understanding of ECP, an unhealthy form of perfectionism characterized by self-criticism and inability to be satisfied with one's performance (Dunkley et al., 2000). Therefore, it was predicted that ECP would significantly predict raising the bar in all weight-related goals, such that higher ECP scores would predict a significant increase in goals on Week 1 (Day 1 to Day 8) and Week 2 (Day 8 to Day 15).

6. As in hypothesis 3 , the study investigated whether adaptive perfectionism, as measured by PSP, significantly predicted raising the bar in all weight related goals (i.e., weight loss, calorie consumption, exercise, and healthy habit goals), such that higher scores on the PSP scale would predict a significant increase in goals on Week 1 (Day 1 to Day 8) and Week 2 (Day 8 to Day 15). 
7. It was predicted that goal-specific self-efficacy would significantly predict raising the bar in all weight related goals (i.e., weight loss, calorie consumption, exercise, and healthy habit goals), such that higher scores on goal-specific self-efficacy would predict a significant increase in goals on Week 1 (Day 1 to Day 8) and Week 2 (Day 8 to Day 15).

\section{Regression Hypotheses: Moderation of Goal-Specific Perceived Success and Self-Efficacy}

8. As raising the bar is considered to occur following success (Shafran et al., 2002), it was predicted that goal-specific perceived success would moderate the relationship between clinical perfectionism and raising the bar in all weight related goals (i.e., weight loss, calorie consumption, exercise, and healthy habit goals). Specifically, higher scores in clinical perfectionism would predict a significant increase in goals on Week 1 (Day 1 to Day 8) and Week 2 (Day 8 to Day 15) when perceived success was high, as demonstrated by a significant interaction between clinical perfectionism and goal-specific perceived success.

9. Self-efficacy has been shown to positively impact performance, to be bolstered by success, and to influence goal-setting. Therefore, it was predicted that goal-specific perceived success and self-efficacy would moderate the relationship between clinical perfectionism and raising the bar in all weight related goals (i.e., weight loss, calorie consumption, exercise, and healthy habit goals). Specifically, higher levels of clinical perfectionism would predict a significant increase on Week 1 (Day 1 to Day 8) and Week 2 (Day 8 to Day 15) when goalspecific perceived success and self-efficacy were high, as demonstrated by a three-way interaction between clinical perfectionism, goal-specific perceived success, and self-efficacy. 


\section{Method}

\section{Participants}

Participants were required to be between the ages of 17 and 65 years, have a BMI over $25.0 \mathrm{~kg} / \mathrm{m}^{2}$, and have a smartphone and valid email address. Participants were permitted to engage in other behavioural or dietary weight loss strategies or interventions as long as they did not interfere with the procedure of the current study. The BMI cutoff was selected based on World Health Organization (WHO, 2006) recommendations, which classifies individuals with a BMI of over $25.0 \mathrm{~kg} / \mathrm{m}^{2}$ as overweight. A description of the response rate throughout the study from initial phone screen to completion is provided in Figure 1, including information about eligibility. The final sample for the current study included 66 participants ( 54 females) with a mean age of 27.17 years $(S D=10.64$, range $=17$ to 59$)$. Participants had a mean BMI of 30.77 $\mathrm{kg} / \mathrm{m}^{2}(S D=3.63$, range $=25.1$ to 41.3$)$. Participants were largely well educated, with $76.3 \%(n$ $=51$ ) having completed at least some college or university. The greatest number of participants identified as "White/European" $(n=23,34.8 \%)$ and "Asian" $(n=25,37.9 \%)$, whereas a smaller number identified as "Hispanic/Latin American” ( $n=5,7.6 \%)$, "Middle Eastern” $(n=5,7.6 \%)$, "Black/Afro-Caribbean/African" ( $n=3,4.5 \%)$, "Biracial/multiracial” $(n=3,4.5 \%)$, "Aboriginal" $(n=2,3.0 \%)$, and "Other" $(n=5,7.6 \%)$. Twenty-seven $(40.9 \%)$ participants reported being "single," $23(34.8 \%)$ as "in a steady relationship," $14(21.2 \%)$ as “married/cohabiting," and two (3\%) as "divorced." Over half of participants $(n=39,59.2 \%)$ reported an annual family income of $\$ 60000$ to $\$ 80000$ or less, with 11 (16.7\%) participants unable to report on income. Although participants were recruited throughout the community via posters, online advertising (e.g., Kijiji, Craigslist), and social media (e.g., Reddit, Facebook), participants were largely comprised of postsecondary students, with 50 participants currently 
enrolled in an educational program (48 = university degree, $2=$ adult/continuing education).

\section{Measures}

Clinical Perfectionism Questionnaire (CPQ). The CPQ (Fairburn et al., 2003a), which was the primary measure of perfectionism and predictor variable of raising the bar in the current study, is a 12-item, self-report questionnaire designed to assess clinical perfectionism as defined by Shafran et al. (2002). Specifically, the CPQ measures the tendency to set perfectionistic standards and the impact of failing to meet these standards on one's self-worth (e.g., Over the past month, have you felt a failure as a person because you have not succeeded in meeting your goals?). Items are rated using a 4-point scale ranging from 1 (not at all) to 4 (all of the time). A total score is derived by summing the 12 items. Higher scores represent a greater severity of clinical perfectionism. Although the CPQ was created to reflect a unidimensional construct of clinical perfectionism, preliminary evidence suggests that the CPQ can be reliably divided into two factors: (1) personal standards and (2) emotional concerns and consequences (Egan et al., 2016; Dickie, Surgenor, Wilson, \& McDowall, 2012; Stoeber \& Damien, 2014). However, there is some discrepancy across these studies regarding which items should be retained to create these two factors. When examining standard setting, Egan et al. (2012) used the total score to measure clinical perfectionism. As such, only the CPQ total score was used in the present study. The psychometric properties of the CPQ have been demonstrated in both nonclinical (Chang \& Sanna, 2012; Dickie et al., 2012; Egan et al., 2016; Stoeber \& Damien, 2014) and eating disorder samples (Egan et al., 2016), with the total score and the two factors showing moderate test-retest reliability and internal consistency, and convergent and discriminant validity. Cronbach's $\alpha=.63$ in the present study. Traditionally, the CPQ measures perfectionism outside the domain of eating, weight, and appearance as indicated by the instructions (e.g., In this questionnaire we are 
only concerned with perfectionism that affects areas of life other than your eating, weight, or appearance). Given that the current study was interested in examining clinical perfectionism specifically within the domain of eating and weight loss, these instructions were removed.

Evaluative concerns and personal standards perfectionism. Evaluative concerns perfectionism (ECP) and personal standards perfectionism (PSP; Dunkley et al., 2000; Stoeber \& Otto, 2006) acted as secondary perfectionism measures and predictor variables of raising the bar in the current study. ECP and PSP are composite scores created from subscales of the FMPS (Frost et al., 1990) and the HMPS (Hewitt \& Flett, 1991).

The FMPS is a 35-item self-report questionnaire that assesses six dimensions of perfectionism: (1) concern over mistakes (CM; experiencing anxiety about making mistakes or being a failure), (2) doubts about actions (DA; second guessing the quality of one's action or work), (3) personal standards (PS; having excessively high standards for oneself), (4) parental expectations (PE; believing that one does not live up to his or her parent's expectations), (5) parental criticism (PC; believing that one's parents will criticize him or her for not living up to standards), and (6) organization ( $\mathrm{O}$; intently focusing on establishing precision, order, or organization). Some of these dimensions seem to assess elements or features of perfectionism (e.g., CM, DA, PS, O), and others seem to assess correlates or causes of perfectionism (e.g., PE, PC; Egan et al., 2014). Items are rated on a 5-point scale ranging from 1 (strongly disagree) to 5 (strongly agree). Items in each dimension are summed to create subscale scores. A total score can be derived by summing the scores of all subscales, with the exception of the $\mathrm{O}$ subscale, as this subscale demonstrated poor intercorrelation with the other subscales and with the total score (Frost et al., 1990). Higher scores indicate greater perfectionism. The FMPS has been shown to 
have acceptable convergent validity, discriminant validity and internal consistency for the six subscales with Cronbach's $\alpha$ ranging from .77 to .93 (Frost et al., 1990).

The HMPS is a 45-item self-report measure that assesses three dimensions of perfectionism: (1) self-oriented perfectionism (SOP; setting excessively high standards for oneself and being overly critical of one's performance), (2) other-oriented perfectionism (OOP; having excessively high standards for others and being overly critical of their performance, and (3) socially prescribed perfectionism (SPP; believing that significant others have excessively high standards for oneself, and placing importance on meeting those standards). Items are rated on a 7-point scale ranging from 1 (strongly disagree) to 7 (strongly agree). Subscale scores can be obtained by summing the items for each subscale. Higher scores represent greater perfectionism. The HMPS has demonstrated adequate test-retest reliability $(r=.60-.69)$ and good internal consistency for each subscale, with Cronbach's $\alpha$ ranging from .82 to .87 . Concurrent validity for the HMPS has been demonstrated in clinical and nonclinical samples, and evidence from a student sample supports its 3-factor structure (Hewitt \& Flett, 1991; Hewitt, Flett, Turnbull-Donovan, \& Mikail, 1991).

ECP is understood as the aspect of perfectionism that involves the perception of unrealistic and negative expectations of others, high levels of self-criticism, and the inability to experience satisfaction upon meeting one's goals, and is computed by summing the $z$-scores of subscales FMPS CM, FMPS DA, and HMPS SP. PSP is understood as the aspect of perfectionism that involves setting personal goals and standards, and is computed by summing the $z$-scores of subscales FMPS PS and HMPS SOP. Overall, the perfectionism literature generally supports the notion that perfectionism is comprised of two distinct forms: healthy perfectionism and unhealthy perfectionism, often referred to as adaptive or maladaptive 
perfectionism (Stoeber \& Otto, 2006). Healthy and unhealthy perfectionism have been labeled in a number of different ways (e.g., Dunkley et al., 2000; Frost et al., 1993; Rice, Ashby, \& Slaney, 1998). Regardless of label or measurement method, findings demonstrate that healthy perfectionism is associated with adaptive characteristics (e.g., conscientiousness, positive affect, active coping styles, achievement) to a greater extent than unhealthy perfectionism regardless of measurement method (Stoeber \& Otto, 2006). Although Frost et al. (1993), and other researchers (e.g., Bieling et al., 2003, 2004) have included all HMPS and FMPS subscales into healthy and unhealthy composite perfectionism scores, more recent recommendations justify the removal of the HMPS OOP subscale (Stoeber 2014, 2015) and the FMPS O, PE, and PC subscales (Frost et al., 1990; Stoeber \& Otto, 2006). Specifically, Stoeber $(2014,2015)$ found that OOP is uniquely associated with negative characteristics such as narcissism, Machiavellianism, aggressive humor, and uncaring traits compared to SOP or SPP. As discussed earlier, Frost et al. (1990) recommended that the $\mathrm{O}$ subscale not be included when calculating overall perfectionism due to its poor intercorrelation with other subscales and the total score. Furthermore, PE and PC can be understood as reflecting antecedent rather than core aspects of perfectionism (Stoeber \& Otto, 2006).

Given that clinical perfectionism is conceptualized as a dysfunctional, unhealthy form of perfectionism (Shafran et al., 2002), it was of interest in the current study to see whether convergence would be found between the CPQ and ECP. Furthermore, Bieling et al. (2003) found that adaptive perfectionism, but not maladaptive perfectionism, predicted raising the bar. This in contrast to what might be expected in the context of the clinical perfectionism model.

Eating Disorders Examination - Questionnaire (EDE-Q). Originally developed as an investigator-based interview (Cooper \& Fairburn, 1987), the EDE-Q (Fairburn \& Bèglin, 1994) 
was reformatted into a 28-item self-report questionnaire. Both formats were designed to assess the severity of eating disorder psychopathology over the past 28 days. The EDE-Q includes four cognitive subscales or features of eating disorders: (1) dietary restraint, (2) eating concern, (3) weight concern, and (4) shape concern. There is some debate in the literature over the appropriate organization of these factors (Hrabosky, White, Masheb, Rothschild, BurkeMartindale, \& Grilo, 2008; Peterson et al., 2007), and whether the cognitive subscales are better represented by one overarching dimension of eating disorder psychopathology (Aardoom, Dingmans, Slof Op't Landt, \& Van Furth, 2012). The measure also includes items that address the frequency of a number of behavioural features of eating disorders including binge eating, self-induced vomiting, misuse of laxatives or diuretics, and excessive exercise. Items are rated based on the frequency of behaviours or cognitions on a 7-point scale ranging from 0 (no days) to 6 (everyday), or from 0 (not at all) to 6 (markedly), depending on the item. A separate set of items ask the individual to indicate the number of times he or she has engaged in a behaviour over the previous 28 days. However, these items are not included in the total or subscale scores, and are instead used as a diagnostic assessment tool. In a systematic review of the psychometric properties of the EDE-Q (Berg, Peterson, Frazier, \& Crow, 2012), investigators reported adequate test-retest reliability for the four cognitive subscales and behavioural items, and fair to excellent internal consistency, with Cronbach's $\alpha$ ranging from .70 to .93 for the four cognitive subscales. There is some evidence for convergent validity for the full scale (Fairburn \& Beglin, 1994) and for the dietary restraint subscale (Bardone-Cone \& Boyd, 2007), however research is still needed to establish the validity for the other three subscales as well as the behavioural frequency items (Berg et al., 2012). In the present study, Cronbach's $\alpha$ for the total and subscale scores ranged from .80 to .93 . 
Accurately identifying an episode of binge eating, as defined by the fifth edition of the Diagnostic and Statistical Manual of Mental Disorders (DSM-5, American Psychological Association, 2013), is one of the biggest challenges in the assessment of eating pathology (Anderson, Lundgren, Shapiro, \& Paulosky, 2004). Research shows that individuals may incorrectly use the term "binge eating" when experiencing a loss of eating control even when a normal amount of food was consumed. However, both of these elements are necessary for the diagnosis of a binge eating episode (Anderson et al., 2004; Telch, Pratt, \& Niego, 1998).

Therefore, for the current study, a description and examples of binge eating episode was included at the beginning of the questionnaire to inform participants how to identify binge eating in a consistent, systematic manner.

Depression Anxiety Stress Scales - 21 item version (DASS-21). The DASS-21 is the short form of the original 42-item self-report measure of the Depression Anxiety Stress Scales (Lovibond \& Lovibond, 1995a) designed to assess emotional states of depression (e.g., I couldn't seem to experience any positive feeling at all), anxiety (e.g., I felt I was close to panic), and stress (e.g., I found it hard to wind down) over the previous week. The DASS-21 has several advantages compared to the full version, including smaller interfactor correlations, fewer items, and a neater factor structure (Antony, Bieling, Cox, Enns, \& Swinson, 1998). Specifically, the factor structure of the DASS-21 has been supported in both clinical and nonclinical samples (Clara, Cox, \& Enns, 2001; Sinclair et al., 2012). Items are rated using a 4-point scale ranging from 0 (did not apply at all) to 3 (applied to me very much). When scoring the DASS-21, items in each subscale are summed. Total subscale scores are then doubled in order to be comparable to the full version. The DASS-21 has been shown to have good convergent and discriminant validity and excellent internal consistency, with Cronbach's $\alpha$ ranging from .84 to .94 in both 
clinical and nonclinical samples (Antony et al., 1998; Sinclair et al., 2012). In the present study, Cronbach's $\alpha$ ranged from .82 to .93 .

Eating Self-Efficacy Scale (ESES). The ESES (Glynn \& Ruderman, 1986) was developed primarily for use by individuals who are overweight or dieting. It is comprised of 25 self-report items designed to assess the perceived ability to control overeating in a number of different situations (e.g., after work or school, when preparing food, when with friends or family) or during certain emotional states (e.g., when angry, tense, or annoyed). These two factors have been labeled socially acceptable circumstances (SAC) and negative affect (NA) respectively. Total and factor scores are created by summing items. The current study used the total score to calculate general eating self-efficacy. Items are rated using a 7-point Likert scale ranging from 1 (no difficulty controlling eating) to 7 (most difficulty controlling eating). Therefore, lower scores represent higher levels of eating self-efficacy. The ESES has demonstrated good convergent validity, acceptable test-retest reliability $(r=.70, p<.001)$, and good internal consistency for the total score, with Cronbach's $\alpha=.92$ (Glynn \& Ruderman, 1986). Cronbach's $\alpha=.94$ in the present study.

My Fitness Pal ${ }^{\circledR}$ (MFP) Food and Exercise Diary. MFP is a free self-monitoring food and exercise diary smartphone app used to track energy consumption and expenditure, and is compatible with iPhone, iPad, Android, Blackberry, and Windows smartphones. Users create an account with a valid email address, and receive a suggested number of calories to consume based on their BMI and desired weekly weight loss goal ( $0.5-2$ pounds [lbs.] per week). Daily caloric consumption and exercise can be tracked using the smartphone app or by logging on at www.myfitnesspal.com. To track caloric consumption, users enter any food item consumed into a searchable food database of over 4 million items, which provides a number of potential food 
matches complete with nutritional facts (e.g., calories, fat [g], cholesterol [mg], sodium [mg], carbohydrates $[\mathrm{g}]$ ). The MFP database recognizes regular food items (e.g., apple, rye bread), meals (e.g., Pad Thai, spaghetti with meat sauce), or food brands or restaurants (e.g., Swiss Chalet, homemade, Heinz), and provides a number of relevant options after entering an item into the search bar. A similar database exists to track exercise. MFP does not require Internet access or mobile data to search food items or to update the food and exercise diary. However, when connected to WiFi or mobile data, MFP syncs smartphone app information with the online account at www.myfitnesspal.com. MFP offers a number of features to facilitate tracking, including daily reminders, progress charts and reports, diary sharing, and personalized databases to $\log$ frequent foods or exercises more easily.

Goal-Setting Questionnaire (GSQ). The GSQ was designed for the current study to assess the construct of raising the bar for weight-related goals pertaining to MFP tracking and weight loss. The GSQ includes four goal domains: (1) weight loss goals (WLG), (2) calorie consumption goals (CCG), (3) exercise goals (EG), and (4) healthy habit goals (HHG). Each of these goal domains includes a measure of (1) raising the bar, (2) goal-specific self-efficacy and (3) goal-specific perceived success. Raising the bar was measured by calculating actual changes in each of the four goal domains at two times points: Week 1 (Day 1 to Day 8), and week 2 (Day 8 to Day 15). For example, if a participant set a WLG of $1.5 \mathrm{lbs}$. on Day 1, and $2 \mathrm{lbs}$. on Day 8 , then the change score for Week 1 would equal $0.5 \mathrm{lbs}$. An increase in goals represented raising the bar.

Goal-specific self-efficacy was measured for each goal domain at each time point (Day 1, Day 8, Day 15) by asking participants to indicate on a 7-point scale ranging from 1 (not at all successful) to 7 (completely successful), the extent to which they expect to be successful in 
meeting their goal the upcoming week. Goal-specific perceived success was measured for each goal domain at two time points (Day 8, Day 15) by asking participants to reflect back on the previous week and indicate how successful they were at meeting their goal. This item used the same 7-point scale as goal-specific self-efficacy.

$\boldsymbol{W L G}$. For WLG, participants were asked how many pounds they would like to lose per week. This goal domain was split into two parts: (1) A MFP WLG and (2) a personal WLG. As MFP restricts individuals to four options ranging between $0.5 \mathrm{lbs}$. per week to $2 \mathrm{lbs}$. per week, the personal WLG asked participants (1) if they would set a different WLG than MFP, and (2) if yes, what that WLG would be. The personal WLG was implemented to avoid a possible ceiling effect on the number of pounds participants wished to lose per week. For the current study, only the MFP WLG was analyzed as the minority of participants chose to set a personal WLG, leading to insufficient sample size for the change score analysis for Week $1(n=25)$ and for Week $2(n=19)$. Raising the bar was represented by a larger number of weekly pounds selected on Day 8 compared to Day 1, or on Day 15 compared to Day 8.

CCG. MFP provides participants with a suggested number of calories to consume per day based on their BMI, weekly weight loss goal, and total overall weight loss goal. For CCG, participants were asked how many days during the upcoming week they would like to be at or below the suggested number of MFP calories. Raising the bar was represented by a larger number of days on Day 8 compared to Day 1, or on Day 15 compared to Day 8.

$\boldsymbol{E G}$. For EG, participants were asked to indicate the amount of exercise they would like to complete during the upcoming week by selecting the number of days, and the duration (in minutes) of each exercise session. Participants were also asked to indicate the type of exercise (e.g., running, swimming, weight training, etc.). Raising the bar was represented by a larger 
number of total weekly exercise minutes on Day 8 compared to Day 1, or on Day 15 compared to Day 8.

HHG. For HHG, participants were asked to select a number of healthy behaviours they would like to adopt for the week (e.g., avoid distractions while eating, drink water instead of sugary drinks, or eat one dark green and one orange vegetable per day). Participants were able to choose as many HHG as they wanted from a list of 15 goals, as well as add additional goals not mentioned in the list. Raising the bar was represented by a larger number of total healthy habit goals selected on Day 8 compared to Day 1, or on Day 15 compared to Day 8. See Appendix A.

General Goal-setting Questionnaire (GGSQ). The GGSQ (Krause et al., 2016) is a 9item self-report measure designed to assess the general tendency of individuals to raise the bar in any relevant goal-setting domain. The questionnaire provides a brief description of goal-setting and examples of different domains in which individuals might set goals. Krause et al. (2016) found that the scale is best captured by one higher order factor (i.e., total score) and two lowerorder factors: Standard Dissatisfaction (GGSQ StanD), the dissatisfaction with success and reappraisal of successful goals as being insufficiently challenging (e.g., "Meeting my goal means that it must have been too easy") and Standard Increase (GGSQ StanI), the subsequent setting of more challenging goals (e.g., "I move onto more challenging or difficult goals after meeting a goal”). Items are rated on a 7-point scale, ranging from 1 (strongly disagree) to 7 (strongly agree) and summed to create total and factor scores. Higher scores represent a stronger tendency to raise the bar. Krause et al. (2016) found good test-retest reliability and internal consistency for the total and factor scores, with Cronbach's $\alpha$ for the total and factor scores ranged from .87 to 93. Higher CPQ scores were shown to significantly predict an increase in total and factor scores (Krause et al., 2016). In the present study, Cronbach's $\alpha$ ranged from .80 to .90. See Appendix B. 


\section{Procedure}

The present study involved three weekly in-person lab visits over the course of 14 consecutive days of MFP food and exercise tracking. Participants were tested individually by one of two experimenters. Phone or email screens were conducted to ascertain eligibility (e.g., appropriate BMI, age, smartphone, email, etc.). As weight-related goal domains were created to measure goals for a period of 7 days, in-person lab visits were scheduled to occur at the same time exactly 1 week apart (e.g., Monday at 9:00). If participants needed to reschedule, they were permitted to come in either 1 day before or after the 1 week time mark so as to not interfere with weight-related goal-setting or ratings of goal-specific perceived success and self-efficacy. For the second lab visit, five (7.6\%) participants were scheduled 1 day after the 1 week time mark. For the third lab visit, five $(7.6 \%)$ participants were scheduled 1 day after the 1 week time mark and $4(6.1 \%)$ were scheduled 1 day before the 1 week time mark.

First Lab visit. On Day 1, participants completed the first in-person 1-hour lab visit. First, informed consent was obtained. Next, participants' BMI was objectively determined by measuring baseline weight and height using a digital scale and measuring tape. In the rare case where a participant's BMI was at or under $25.0 \mathrm{~kg} / \mathrm{m}^{2}$, participants were compensated $\$ 10$ for attending the first meeting, and provided with some brief psychoeducation about their BMI and weight falling within a healthy range. Next, participants completed the questionnaire battery (CPQ, FMPS, HMPS, EDE-Q, DASS-21, and ESES) using Qualtrics online software. Each participant was assigned a nonidentifying questionnaire user ID for all study materials. After completing the questionnaires, participants were provided information on the efficacy of selfmonitoring and goal-setting in behavioural weight loss programs. During this discussion, participants were instructed to abstain from weighing themselves in-between lab visits for the 
duration of the study. Given that weight fluctuates on a daily basis, weighing oneself weekly is a general recommendation in cognitive-behavioural eating disorder and weight interventions (e.g., Fairburn, 2013). Next, participants created an MFP account using a personal email, by logging onto www.myfitnesspal.com with the experimenter. As MFP accounts require the creation of a username, a separate MFP user ID was created so as to not connect identifying information (i.e., the participant's email) with the questionnaire user ID. In order to link questionnaire data with MFP data, the participant's email, questionnaire user ID, and MFP user ID were kept in a password protected document only accessible to the researchers involved in the study. Participants received thorough instruction on using MFP (e.g., navigating the app, entering food and exercise) and were given the option of setting reminders in their phones to facilitate daily self-monitoring. Finally, participants were introduced to and completed the GSQ. The experimenter left the room while participants completed questionnaires (e.g., Qualtrics, GSQ, etc.). Participants received a hard copy of their weight-related Day 1 goals, as completed on the GSQ, to take home as a reminder of their intended goals throughout the week. See Appendix C.

Second Lab Visit. On Day 8, participants returned for a 20-minute lab visit. Participants were asked if they had weighed themselves during the week, and were weighed using the same electronic scale as Day 1 to determine actual weight loss. Participants then logged onto their MFP account on a lab computer, and the experimenter documented their net calories and total exercise minutes each day on a separate sheet of paper. Participants were then provided with the summary of their weight-related Day 1 goals. This summary enabled them to rate perceived success while completing the GSQ a second time. Participants again received a hard copy of their new weight-related Day 8 goals to take home as a reminder of their intended goals throughout the week. Participants received \$10 for coming to the second lab visit. 
Third Lab Visit. On Day 15, participants returned for a final 20-minute lab visit. Participants were asked if they weighed themselves during the week, and were weighed using the same electronic scale as Day 1 and Day 8 to determine actual weight loss. Participants then logged onto their MFP account on a lab computer, and the experimenter tracked their net calories and total exercise minutes each day on a separate sheet of paper. Participants were then provided with the summary of their weight-related Day 8 goals, and completed the GSQ a third time. Following completion of the GSQ, participants completed the GGSQ using the online Qualtrics software. The following open-ended question was also included in the Qualtrics questionnaire: What factors determine whether you decide to set a more challenging goal for yourself after successfully meeting a previous goal? Responses to this question were used for a posthoc qualitative analysis. Finally, participants were fully debriefed and received $\$ 10$ for coming to the third lab visit. 


\section{Data Analytic Strategy}

\section{Descriptive Analyses}

Before conducting any analyses, raw data were screened for normality, outliers, homogeneity of variance, multicollinearity, homoscedasticity, and missing data. All missing values were replaced using series mean substitution, and outliers were identified by transforming total scores into $z$-scores. Based on the normal distribution, one would expect all standardized values to fall within the absolute value of 3.29 (Tabachnick \& Fidell, 2007), and 1\% of values to exceed the absolute value of 2.58. Outliers for weight-related goals were not removed given that goals set are meant to be self-imposed and personally demanding. Any parametric violations or problems were addressed prior to conducting any further statistical tests. Statistical significance was set a priori at less than or equal to $p=.05$.

\section{Correlational Analyses: Relationship Between Perfectionism, Self-Efficacy, and Raising the}

\section{Bar}

Correlational analyses were used to test Hypothesis 1. The CPQ, ECP, PSP, GGSQ total and factor scores, ESES, DASS anxiety, stress, and depression, and the EDEQ total score were entered into a bivariate correlational matrix, using two-way Pearson $r$ correlation coefficients. The DASS and EDEQ were entered for descriptive purposes to identify the extent to which these measures of psychopathology were related to perfectionism, raising the bar, and self-efficacy.

\section{Regression Analyses: Predicting the General Tendency to Raise the Bar}

A series of linear regressions were used to test Hypotheses 2 through 3 . The CPQ, ECP, PSP, and ESES were entered into separate models to predict GGSQ total, StanD and StanI scores, creating a total of 12 linear regressions.

\section{Regression Analyses: Predicting Weight-Related Raising the Bar}


A series of moderated hierarchical regressions were used to test Hypotheses 4 through 9. Raising the bar was measured for each weight-related goal domain (i.e., WLG, CCG, EG, HHG) by calculating the actual change in goals for Week 1 (Day 8-Day 1) and for Week 2 (Day 15-Day 8). A significant increase in goal difficulty on Day 8 compared to Day 1, or Day 15 compared to Day 8 (e.g., more weekly weight loss, calorie consumption days, exercise minutes, or healthy habit goals) represented raising the bar. A separate hierarchical regression was conducted for each weight-related goal (i.e., WLG, CCG, EG, HHG) and time where raising the bar was measured (i.e., Week 1 and Week 2). This created a total of eight regressions (4 goal domains x 2 raising the bar measurements) for each measure of perfectionism (i.e., CPQ, ECP, PSP).

Goal-specific self-efficacy for Week 1 was measured by averaging the goal-specific selfefficacy score of Day 1 and Day 8 for each respective goal domain. Self-efficacy for Week 2 was measured by averaging the goal-specific self-efficacy score of Day 8 and Day 15. As participants only rated their goal-specific perceived success on Day 8 and Day 15, success for Week 1 was measured by using the Day 8 goal-specific perceived success score for each respective goal domain. Success for Week 2 was measured by using the Day 15 goal-specific perceived success score for each respective goal domain. Therefore, a separate measure of goal-specific selfefficacy and perceived success was used for each of the eight hierarchical regressions. Three independent predictors were entered using forced entry into Step 1 of each hierarchical regression: (1) perfectionism (i.e., CPQ, ECP, or PSP), (2) goal-specific self-efficacy, and (3) goal-specific perceived success. These main effects were used to test the hypotheses that perfectionism, as measured by the CPQ (Hypothesis 4), ECP (Hypothesis 5), PSP (Hypothesis 6), and goal-specific self-efficacy (Hypothesis 7) significantly predicted raising the bar in weight-related goals. The two-way interaction terms and the three-way interaction terms of these 
independent predictors were entered using forced entry into Step 2 and Step 3 respectively of each hierarchical regression to see whether goal-specific perceived success, goal-specific selfefficacy, or both moderated the relationship between perfectionism and raising the bar (Hypothesis 8 and 9).

To account for multicollinearity when examining moderation in regression analyses, all single predictors, two-way, and three-way interaction variables were centered on the mean before entering them into the model. To control for violations of normality of errors and homoscedasticity across weight-related goal domains, $\Delta \mathbf{R}^{2}$ and beta values were based on 1000 bootstrap samples. Bias corrected and accelerated (BCa) 95\% confidence intervals (CI) were provided for each unstandardized beta. Significant unstandardized beta coefficients for independent predictors were interpreted only when no significant two-way interaction was present. Similarly, significant two-way unstandardized beta coefficients were interpreted only when no significant three-way interaction was present. Significant beta coefficients for interactions were evaluated using simple slopes analysis (Aiken \& West, 1991). Slopes were evaluated at $1 S D$ above and below the mean of the centered moderator variable (e.g., goalspecific perceived success or self-efficacy). 


\section{Results}

\section{Descriptive Statistics}

Upon examination of the self-report questionnaire data, no $z$-scores were larger than the absolute value of 3.29. One $z$-score exceeded the absolute value of 2.58 for the (1) EDEQ shape concern subscale, (2) DASS anxiety subscale, and (3) GGSQ StanI. The box-plot distribution of these scales did not identify these values as outliers. Therefore, they were not removed from analyses. Two values exceeded the absolute value of 2.58 for the GGSQ StanD and were identified as outliers on the boxplot distribution of this scale. Removing these values from analyses had no effect on results for GGSQ StanD. Therefore, they were not removed from analyses. Due to a procedural error, one participant completed the GGSQ following debriefing and was removed from analyses. As such, analyses involving the GGSQ total or factor scores are based on a sample of 65 completed participants.

Using independent samples $t$-tests and chi-square tests, comparisons between study completers $(n=66)$ and those that dropped out or were removed from the final analysis $(n=20)$ revealed no significant differences between groups on age, gender, ethnicity, education, relationship status, income, GGSQ total or factor scores, ECP, DASS anxiety or depression, ESES, or EDEQ eating, weight, or shape concern. Participants who completed the study were significantly higher than those who dropped out or were removed from the final analysis on the CPQ, PSP, EDEQ dietary restraint subscale, and DASS stress subscale (See Table 1). The mean and standard deviation of the completer sample on primary measures compared to the total possible score on that measure are presented in Table 2. The mean, standard deviation, minimum, maximum, and mode for weight-related goals on each day are presented in Table 3. Additionally, examining the distribution of weight-related goals using histograms and boxplots 
revealed a negative skew for WLG and CCG and a positive skew for EG and HHG on Day 1, Day 8, and Day 15 (Plots not shown). Five outliers were identified for EG on Day 1 (Total exercise minutes $=420,475,540,630,900)$, and 2 outliers for EG on both Day 8 (Total exercise minutes $=475,540)$ and Day $15($ Total exercise minutes $=475,525)$. These outliers were not removed in subsequent analyses given that weight-related goals are meant to be self-imposed and personally demanding. No outliers were found for the WLG, CCG, or EG. Clinical perfectionism was not significantly correlated with WLG, CCG, EG, or HHG on Day 1, Day 8, or Day 15 ( $r$ ranging from .001 to .178). Given the parametric violations when conducting a one-way analysis of variance (ANOVA; i.e., normality, sphericity), a nonparametric Friedman's ANOVA was used to calculate whether weight-related goals (i.e., WLG, CCG, EG, HHG) increased or decreased significantly over time. Friedman's ANOVA was nonsignificant for WLG, $\chi^{2}(2)=$ $4.51, p=.105, \mathrm{CCG}, \chi^{2}(2)=2.24, p=.326$, and EG, $\chi^{2}(2)=5.04, p=.08$, demonstrating that these weight-related goals did not significantly increase or decrease across Day 1, Day 8, and Day 15. In contrast, Friedman's ANOVA was significant for HHG, $\chi^{2}(2)=9.93, p=.007$. Stepwise comparisons revealed that, on average, participants selected significantly less HHG on Day 15 than on Day $1, T=307.50, r=-.29, p=.001$, or on Day $8, T=249.00, r=-.27, p=.002$ (See Table 3).

Correlational Analyses: Relationship Between Perfectionism, Self-Efficacy, and Raising the Bar

The correlation matrix between all relevant variables for Hypothesis 1 is presented in Table 4. Psychopathology measures (i.e., DASS, EDEQ) are also included in this matrix. First, it was predicted that a positive correlation would be found between the CPQ and the GGSQ total and factor scores. This hypothesis was partially confirmed, with significant positive correlations 
between the CPQ and the GGSQ total and GGSQ StanD, but not for the GGSQ StanI score. Second, it was predicted that a positive correlation would be found between the ESES and GGSQ total and factor scores. This hypothesis was partially confirmed, as demonstrated by a significant positive correlation between ESES and GGSQ total and GGSQ StanD scores, but not the GGSQ StanI score. Third, it was predicted that a negative correlation would be found between the ESES and both the CPQ and ECP. The opposite was found, with significant positive correlations between both the ESES and the CPQ, and the ESES and ECP. Finally, it was predicted that a positive correlation would be found between the ESES and PSP. This prediction was not confirmed. No significant correlations were found between the GGSQ StanI score and any other measures. Furthermore, PSP, unlike all other measures, was not significantly correlated with either the GGSQ total or factor scores.

\section{Regression Analyses: Predicting the General Tendency to Raise the Bar}

For Hypothesis 2, it was predicted that higher scores in perfectionism (CPQ, ECP) and general eating self-efficacy would significantly predict the general tendency to raise the bar, as measured by the GGSQ total and factor scores. Hypothesis 2 was partially supported (See Table 5), such that higher scores on the CPQ, ECP, and ESES predicted an increase in both GGSQ total and StanD, but not StanI scores. This is consistent with a lack of significant correlations between perfectionism and self-efficacy measures and the GGSQ StanI scores. For Hypothesis 3, it was predicted that PSP would also result in a general tendency to raise the bar. This hypothesis was based on Bieling et al. (2003), who found that adaptive perfectionism, but not maladaptive perfectionism significantly predicted setting higher standards for an upcoming exam. Hypothesis 3 was not supported. PSP did not significantly predict GGSQ total or factor scores. This is also in line with the lack of significant correlations between PSP and GGSQ total and factor scores. 


\section{Regression Analyses: Predicting Weight-Related Raising the Bar}

For Hypotheses 4-7, it was predicted that perfectionism (i.e., CPQ, ECP, PSP), and goalspecific self-efficacy would predict a significant increase in goals on Week 1 (Day 1 to Day 8) and Week 2 (Day 8 to Day 15) in all weight-related goals (e.g., WLG, CCG, EG, and HHG). For Hypotheses 8 and 9, it was predicted that goal-specific perceived success and self-efficacy would moderate the relationship between the CPQ and raising the bar. As a further exploratory analysis, it was also examined whether these two-way and three-way interactions resulted in a greater increase in weight-related goal domains when predicted by other measures of perfectionism (i.e., ECP, PSP). Results for these moderated hierarchical regressions are presented in Tables 6 through 11 .

Individual predictors. Hypotheses 4-7 were not supported for any weight-related goal for either Week 1 or Week 2 change scores of raising the bar. Although not included in the current study's hypotheses, goal-specific perceived success as a single predictor of raising the bar in Step 1 was significant in two cases. A one point increase above the mean for CCG success significantly predicted a .28 increase in CCG days for Week 2 (Day 8 to Day 15) when CPQ and CCG self-efficacy are held constant at the mean (See Table 9). This finding converged with the ECP model, which revealed a significant increase of .29 CCG days (See Table 10) when ECP and CCG self-efficacy are held constant at the mean.

Two-way interactions. Hypothesis 8 was largely not supported for any weight-related goal for either Week 1 or Week 2 change scores of raising the bar, with the exception of HHG. Examining the two-way interactions between perfectionism and goal-specific perceived success, a significant beta was found for $E C P$ x HHG perceived success on HHG change for Week 1

(Day 1 to Day 8; See Table 7). A one point increase above the mean of ECP $x$ HHG perceived 
success significantly predicted a .22 decrease in HHG change from Day 1 to Day 8 . However, the $95 \%$ BCa CI included zero (-0.47-0.04). Simple slopes analysis (Aiken \& West, 1991) for ECP $x$ HHG perceived success on HHG for Week 1 found that low HHG perceived success ($1.57,1 S D$ below the mean) resulted in a significant upward slope, $b=0.38, t=2.18, p=.033$, such that HHG change scores increased as ECP scores increased (See Figure 2). The slope for high HHG perceived success for ECP Week 1 was not significant (See Table 12).

Examining the two-way interactions between perfectionism and goal-specific selfefficacy, significant betas were found for both HHG change on Week 1 and 2. For HHG Week 1 change, a one point increase above the mean of $C P Q \times H H G$ self-efficacy significantly predicted a .23 increase in HHG selected from Day 1 to Day 8. However, the 95\% BCa CI for HHG included zero (-0.03-0.57), and $\Delta \mathrm{R}^{2}$ of Step 2 did not account for a significant amount of model variance (See Table 6). A similar pattern was found for ECP but not PSP (See Tables 7 and 8 respectively). Simple slopes analysis for Week $1 C P Q \times H H G$ self-efficacy can be found in Figure 3. High HHG self-efficacy $(0.84,1 S D$ above the mean) resulted in a significant upward slope, $b=0.22, t=2.68, p=.01$, such that HHG change scores increased as CPQ scores increased (See Figure 3). The slope for low HHG self-efficacy (-0.84, $1 S D$ below the mean) was not significant (See Table 12). For ECP, both high and low HHG self-efficacy slopes were significant $(b=0.47, t=3.07, p=.003$ and $b=-0.42, t=-2.16, p=.035$, respectively). When HHG self-efficacy was high, HHG change scores increased as ECP scores increased. In contrast, when HHG self-efficacy scores were low, HHG change scores decreased as ECP scores increased (See Figure 4). Slopes for PSP were not evaluated given the lack of significant $\Delta R^{2}$ and betas in the model (See Table 8). 
For HHG Week 2 change, a one point increase above the mean of CPQ $x H H G$ selfefficacy significantly predicted a .23 decrease in HHG change from Day 8 to Day 15 (See Table 9). A similar pattern was found for ECP and PSP (Tables 10 and 11 respectively). For PSP, the 95\% BCa CI for HHG included zero (-0.69-0.14), and the $\Delta \mathrm{R}^{2}$ of Step 2 did not account for a significant amount of model variance. Simple slopes analysis (Aiken \& West, 1991) of the interaction between $C P Q \times H H G$ self-efficacy for Week 2 can be found in Figure 5. High HHG self-efficacy $(0.91,1 S D$ above the mean) resulted in a significant downward slope, $b=-0.15, t=$ $-2.41, p=.019$, such that HHG change scores decreased as CPQ scores increased (See Figure 5). The slope for low HHG self-efficacy (-0.84, $1 S D$ below the mean) was not significant (See Table 12). For ECP, high HHG self-efficacy was significant, $b=-0.24, \mathrm{t}=-2.13, p=.038$, but low HHG self-efficacy was not significant (See Figure 6). When HHG self-efficacy was high, HHG change scores significantly decreased as ECP scores increased. For PSP, the slope for high self-efficacy was not significant (See Table 12), but the slope for low HHG self-efficacy was significant, $b=0.50, t=2.51, p=.015$. When HHG self-efficacy was low, HHG change scores significantly increased as PSP scores increased (See Figure 7). In summary, when HHG selfefficacy was high, HHG change scores decreased as CPQ and ECP increased. When HHG selfefficacy was low, HHG change scores increased as ECP and PSP increased.

Three-way interactions. Hypothesis 9 was not supported, as no significant three-way interactions between the CPQ, goal-specific perceived success, and self-efficacy were found for weight-related goals.

\section{Post Hoc Analysis}

Prediction of total weight loss from perfectionism. Additional post hoc analyses explored whether perfectionism, as measured by CPQ, ECP, and PSP significantly predicted 
overall weight loss. This is a relevant question given that the current study explored perfectionism in a sample that wished to lose weight. Descriptively, the average weight of participants appeared to decrease by approximately $1 \mathrm{lb}$ per week. The mean and standard deviation for completed participants' weight on Day 1, Day 8, and Day 15 was $184.84(S D=$ $29.31), 183.46(S D=28.73)$, and $182.76(S D=28.52)$ respectively. Total weight loss was a variable created to represent the change between participants' weight on Day 1 and their weight on Day 15, such that Day 15 was subtracted from Day 1. A positive score indicated weight lost (e.g., $150-145=5$ pounds lost). A negative score indicated weight gained (e.g., $150-155=-5$ pounds gained). Three linear regressions were run with perfectionism as the predictor and total weight loss as the outcome. Results are presented in Table 13. CPQ was the only significant predictor of total weight loss. A one point increase in the CPQ resulted in a .17 decrease in total weight loss. Therefore, higher levels of perfectionism predicted less weight loss than lower levels of perfectionism.

Qualitative analysis of raising the bar. Shafran et al. (2002) hypothesized that clinical perfectionists raise the bar following success because they reappraise their standard as being insufficiently challenging. In order to identify reasons why individuals raise the bar, participants were asked the following open-ended question in an online Qualtrics questionnaire after completing the GGSQ on Day 15: What factors determine whether you decide to set a more challenging goal for yourself after successfully meeting a previous goal? In order to evaluate factors that lead to raising the bar in individuals who self-report raising the bar, responses provided by participants that scored in the top third (a score of $\geq 14 / 21$ ) on the GGSQ StanI score were included in this analysis. GGSQ StanI includes three questions: (1) When I meet a goal, I set more challenging or difficult goals the next time, (2) I feel the need to set more challenging 
goals after meeting a previous goal, and (3) I move onto more challenging or difficult goals after meeting a goal. It was predicted that there would be a number of reasons contributing to raising the bar, but that participants high in GGSQ StanI and either GGSQ StanD $(\geq 28 / 42)$ or the CPQ ( $\geq 32 / 48)$ would identify reasons for raising the bar that align with Shafran et al.'s (2002) model. Specifically, reasons for raising the bar should refer to a lack of satisfaction with previous goals, the inability to appreciate success, and the motivation to raise the bar in order to avoid failure.

Forty-nine of 66 participants scored in the top third of the GGSQ StanI scale, with 26 participants (49\%) scoring just above the cutoff with a score of 14 to 16 . The second half of participants were relatively evenly distributed among other scores with 5 participants each (10.2\%) scoring 17, 18, and 19, 4 (8.2\%) participants scoring 20, and $6(12.2 \%)$ participants scoring 21. Two of these participants did not provide a response to the open-ended question. Upon examination, open-ended responses fell into five over-arching reasons for raising the bar: (1) Attitude towards the goal, (2) Resources, (3) Difficulty level, (4) Success, and (5) SelfImprovement. The description and examples of each of these reasons can be found in Table 14 . Each phrase or element of a participant's response was coded as corresponding to one of the above categories. In order to establish interrater reliability of these categories, the description in Table 14 was provided to a second coder, blind to the participant's score on the GGSQ StanI and to the initial coding of each statement. Interrater reliability is presented in Table 15.

For the 49 participants who scored in the top third of the GGSQ StanI, the mean and standard deviation of the GGSQ StanD and the CPQ was 20.43 (7.13), and 28.78 (4.95) respectively, demonstrating that on average, participants fell just below half on the GGSQ StanD scale, and just above half on the CPQ. Frequencies of each of these scales are provided in Table 16. Bivariate Pearson $r$ correlations between GGSQ StanI and the GGSQ StanD $(r=-0.73, p$ 
$=.617)$, and the GGSQ StanI and the CPQ $(r=.26, p=.072)$ revealed no significant correlations between the scales.

In order to evaluate the prediction that individuals in the top third of the GGSQ StanI would report themes related to Shafran et al.'s (2002) model of raising the bar particularly when they scored high in the GGSQ StanD, or CPQ, responses for participants (1) in the top third of the GGSQ StanI and (2) in the top third of the GGSQ StanD or the top third of the CPQ scale are presented in Table 17. Interestingly, only two participants scoring in the top third of the GGSQ StanI and either the GGSQ StanD or CPQ gave a reason for the self-improvement category. Given that this category seems to reference setting higher standards most closely, the remaining four statements from this category are presented in Table 18 with their corresponding GGSQ StanD and CPQ scores. While a select few of participants did indicate the need to always aim for higher standards, these statements are not necessarily related to the lack of satisfaction with previously met goals (GGSQ StanD) or with higher clinical perfectionism (CPQ). 


\section{Discussion}

The purpose of the present study was to examine the phenomenon of raising the bar in clinical perfectionism (Shafran et al., 2002). Previous research has evaluated raising the bar using specific experimental tasks (e.g., Stroop task, Kobori et al., 2009; anagram task, Lo \& Abbott, 2013a), in artificial laboratory settings. These past experimental designs deviate from Shafran et al.'s (2002) conceptualization of perfectionism as the stringent pursuit of standards that are (1) in a highly salient domain (e.g., weight, work, school), (2) personally demanding, and (3) self-imposed. Fairburn et al. (2003b) have identified clinical perfectionism as a maintaining mechanism of eating pathology. Therefore, it was deemed appropriate to examine raising the bar in the domain of eating and weight loss. Furthermore, in order to ensure that standards were salient, personally demanding, and self-imposed, only individuals who were overweight or obese and wished to lose weight were recruited for the present study. Eligible participants completed a 2-week self-monitoring and goal-setting study where they (1) tracked their eating and exercise on a smartphone app, and (2) set personal weight-related goals on Day 1, Day 8, and Day 15. It was hypothesized that higher levels of clinical perfectionism would significantly predict both the general tendency to raise the bar, as measured by the GGSQ, as well as a greater increase in four weight-related goals from Day 1 to Day 8, and from Day 8 to Day 15. Furthermore, it was hypothesized that goal-specific perceived success and self-efficacy would moderate the relationship between clinical perfectionism and raising the bar in weight-related goals, such that those higher in clinical perfectionism would raise the bar when goal-specific perceived success and self-efficacy were high. 


\section{Brief Summary of Results}

A number of important results emerged from the present study. First, participants who completed the study protocol had significantly greater clinical perfectionism, PSP, dietary restraint, and stress than participants who dropped out from the study. Second, general eating self-efficacy was positively associated with both clinical perfectionism and ECP, but not PSP. Third, clinical perfectionism, ECP, and general eating self-efficacy were positively associated with and significantly predicted the GGSQ total score. This pattern was also found when examining the relationship of these three variables to the specific tendency to feel dissatisfied with success and reappraise the goal as insufficient, as measured by the GGSQ StanD. However, neither type of perfectionism nor general eating self-efficacy were related to or significantly predicted the specific tendency to set more challenging goals following success, as measured by the GGSQ StanI. PSP was not predictive of the GGSQ total or factor scores. Fourth, there was no consistent predictive pattern of weight-related goals from perfectionism, goal-specific selfefficacy, and success. Higher levels of perfectionism (i.e., CPQ, ECP, PSP) and goal-specific self-efficacy did not predict a greater increase in weight-related goals at either time point. However, goal-specific perceived success significantly predicted raising the bar, as demonstrated by an increase in CCG. While goal-specific perceived success and self-efficacy did not moderate the relationship between perfectionism and raising the bar when evaluated together, each of these variables interacted with perfectionism independently in HHG. These patterns were not consistent across time points, weight-related goals, measures of perfectionism, or level of the moderator. Fifth, post hoc analyses revealed (1) that those higher in clinical perfectionism lost less weight overall than those lower in perfectionism, and (2) individuals scoring high in the specific tendency to set more challenging goals following success, as measured by the GGSQ 
StanI, identified a variety of reasons that contribute to their process of raising the bar following success. However, examination of these reasons overall did not align with Shafran et al.'s (2002) hypothesis that raising the bar occurs due to standard dissatisfaction and reappraisal.

An interpretation of these results is provided within the context of Shafran et al.'s (2002) cognitive-behavioural conceptualization of clinical perfectionism and existing research on standard setting and raising the bar in perfectionism. Following this interpretation is a description of the implications of these findings and recommendations for future research, followed by an explanation of the study's limitations.

\section{Interpretation of Results}

\section{Higher levels of perfectionism, stress, and dietary restraint in completers.}

Participants who completed the study were significantly higher in clinical perfectionism, PSP, dietary restraint, and stress than individuals who dropped out at some point during the study. Completing a 14-day self-monitoring and goal-setting protocol requires a certain level of commitment, motivation, and determination. Given that only participants who wished to lose weight were recruited for the study, participants likely began the study with at least some motivation to engage in behavioural weight change. However, it follows that higher levels of clinical perfectionism may have led to greater motivation and higher rates of completion, as this construct is characterized by the continual striving to meet personally challenging standards, high levels of determination and self-control, and the motivation to avoid failure (Shafran et al., 2002). However, the need to avoid failure in clinical perfectionism is thought to sometimes engender procrastination, abandonment, or avoidance of the task (Shafran et al., 2002). Participants were encouraged to continue to self-monitor and set goals past the 2-week study period. It may be that avoidance or abandonment of the task would have been more apparent had 
the study continued for a longer period of time. For example, while clinical perfectionists are initially more motivated than nonperfectionists to engage in a weight loss program, this significant difference may disappear after longer periods of time (i.e., 1 month).

Personal standards perfectionism is a composite score comprised of the HMPS SOP subscale and FMPS PS subscale, both of which capture the tendency to set excessively high standards for the self. In general, SOP has emerged as a factor related more closely to individuals with eating pathology than with anxiety or depression (Castro-Fornieles et al., 2007) or with healthy controls (Bastiani, Rao, Weltzin, \& Kaye, 1995). Using a nonclinical female university sample, Bardone-Cone (2007) found that SOP, but not SSP, significantly predicted higher rates of dieting and food restraint. Dietary restraint in the present study was defined by the number of days over the past month that individuals had attempted to influence their weight or body shape by (1) limiting food intake, (2) refusing desired foods, (3) following strict rules (e.g., calorie limit), or (4) having an empty stomach. Dietary restraint was measured before beginning any self-monitoring or goal-setting using MFP, and therefore was not influenced by behavioural change related to the study. Given that using MFP requires participants to engage in behaviours related to dietary restraint (e.g., calorie counting), it may be that participants higher in dietary restraint were more amenable to the requirements of MFP than those low in dietary restraint, and therefore more likely to complete the study. Furthermore, given that SOP is related to dietary restraint and eating pathology, it follows that completers higher in dietary restraint would also be higher in PSP than those who dropped out.

On average, participants who completed the study fell within the extremely severe range of anxiety, stress, and depression (Lovibond \& Lovibond, 1995b). This was not significantly different from those who dropped out, with the exception of the stress scale. In spite of this 
statistically significant difference between completers and dropouts on stress, the clinical relevance of this difference is questionable, as participants who dropped out still had clinically elevated levels of stress, with the average falling just above the cutoff score for severe stress. The elevated levels of anxiety, stress, and depression are of interest as past research suggests a positive association between weight loss variables and psychological distress. For example, higher overall BMI (Kubzansky, Gilthorpe, \& Goodman, 2014), the perception that one is overweight (Atlantis and Ball, 2008), and body dissatisfaction (Johnson \& Wardle, 2005) were related to higher levels of psychological distress (e.g., anxiety, stress, depression). Furthermore, weight loss behaviour has been shown to be a significant predictor of future life stress (Rosen, Tacy, \& Howell, 1990). Participants in the present study, whether having completed the study or not, are also characterized by these weight loss variables. For example, recruitment of participants who wish to lose weight may represent a set of individuals who perceive themselves as overweight or are dissatisfied with their body. The relationship between weight loss and higher levels of distress could explain the elevated levels of anxiety, stress, and depression in the entire sample.

2. General eating self-efficacy and perfectionism. It was hypothesized that general eating self-efficacy would be negatively associated with clinical perfectionism and ECP, but positively associated with PSP. The current study found the opposite pattern of results. A positive rather than negative association was found between general eating self-efficacy and both clinical perfectionism and ECP. Furthermore, general eating self-efficacy was not related to PSP. In support of the present study's hypotheses, Burns (1980) proposed that perfectionism should undermine self-efficacy, as excessive standards of perfectionists prevent success, which in turn lowers self-efficacy. Moreover, Dunkley et al. (2000) revealed that the relationship 
between ECP and distress is mediated by daily hassles and maladaptive, avoidant coping strategies (e.g., denial, withdrawal), whereas the relationship between PSP and distress is mediated by daily hassles and active coping strategies. Therefore, while both types of perfectionists experience stress, it is suggested that those higher in ECP engage in more dysfunctional coping strategies than those higher in PSP. Conceptually, dysfunctional coping is thought to lead to a helpless orientation (Dunkley et al., 2000; Flett, Hewitt, Blankstein, and Mosher, 1995), resulting in lower levels of self-efficacy (Dunkley et al., 2000). Lo and Abbott (2013a) found that adaptive perfectionists self-reported higher levels of self-efficacy than maladaptive perfectionists or nonperfectionists, as measured by the APS-R (Slaney et al., 2001). This research supports the hypothesis that adaptive perfectionism should be positively associated with self-efficacy and maladaptive perfectionism negatively associated with self-efficacy.

In contrast, Bandura (1986) suggested that the high expectations of the perfectionist may improve performance, and therefore bolster self-efficacy. Hart et al. (1998) found that SOP, a subscale included in PSP, was negatively associated with self-efficacy whereas SPP, a subscale included in ECP, was positively associated with self-efficacy. The reason for the discrepancy between hypotheses and findings remains unclear. Overall, PSP was unrelated to the majority of variables in the study, which could explain its lack of association with general eating selfefficacy. Conceptually, it may be that individuals who are overweight and wish to lose weight have a higher level of general eating self-efficacy than individuals who are overweight and do not wish to make any behavioural changes. Therefore, elevated self-efficacy may have been required to get participants to sign up for the study and elevated perfectionism may have been required to complete the study, resulting in significant associations between self-efficacy, clinical perfectionism, and ECP. 
3. General tendency to raise the bar (GGSQ). It was hypothesized that perfectionism and general eating self-efficacy would significantly predict the general tendency to raise the bar, as measured by the GGSQ total and factor scores. While individuals can set more challenging goals for a variety of reasons, setting more challenging goals (i.e., GGSQ StanI) in clinical perfectionism is thought to follow dissatisfaction with success and subsequent reappraisal that the standard is insufficient (i.e., GGSQ StanD; Shafran et al., 2002). Results of the present study revealed that clinical perfectionism significantly predicted the GGSQ total and GGSQ StanD scores, but not the GGSQ StanI score. These findings suggest that while clinical perfectionism may be related to the emotional experience of dissatisfaction (e.g., My sense of accomplishment never lasts long after meeting a goal) and the cognitive reappraisal of the goal as insufficient (e.g., Meeting my goal means that it must have been too easy), it is not related with the actual follow-through of setting more challenging goals (e.g. I move onto more challenging or difficult goals after meeting a goal). From a psychometric perspective, the ability of clinical perfectionism to predict the total score is likely more strongly influenced by GGSQ StanD items than StanI items, still suggesting that perfectionism leads to standard dissatisfaction and reappraisal, but not raising the bar.

Results for ECP were the same as those for clinical perfectionism. However, PSP was not significantly related with and did not significantly predict the GGSQ total and factor scores. PSP is characterized by a stringent focus on the need to attain perfectionism for the self, rather than a lack of satisfaction upon success (Dunkley et al., 2002). This might explain the lack of significance between PSP and the GGSQ total and StanD scores. It is also not surprising to find null results between PSP and GGSQ StanI, given the lack of association between GGSQ StanI and any other measure used in the study. ECP and PSP were included in the current study based 
on findings from Bieling et al. (2003), the only study to evaluate the influence of perfectionism on standard setting in a salient life domain. Given that Bieling et al. (2003) did not reference Shafran et al. (2002) or include a specific measure of raising the bar in their study, the present study attempted to further these findings by evaluating the role played by adaptive and maladaptive perfectionism in raising the bar. Bieling et al. (2003) found that adaptive perfectionism, but not maladaptive perfectionism, as measured using composite scores from Frost et al. (1993), significantly predicted setting higher future exam standards over and above one's success or failure in meeting a standard for a previous midterm. Although adaptive and maladaptive perfectionism (Frost et al., 1993) used in Bieling et al. (2003) include more FMPS and HMPS subscales than PSP or ECP, using Frost et al.'s (1993) composite scores revealed the same pattern of results as Table 5, except with smaller beta values (Results not shown). This suggests that the PSP and ECP are comparable and perhaps more streamlined than Frost et al.'s (1993) measures of adaptive and maladaptive perfectionism.

Future exam standards in Bieling et al. (2003) were quantified by asking participants to identify the percentage grade that would be acceptable to them or make them happy, but did not involve a comparison between the previous exam standard and the future exam standard. While similar, future exam standards is not entirely synonymous with the tendency to raise the bar as measured by the GGSQ. Raising the bar requires (1) the comparison of a current goal to a previous goal, and (2) that the current goal be more challenging than the previous goal. Given this lack of comparison, future exam standards is better understood as an independent future goal than a measure of raising the bar. Therefore, findings in the present study show the opposite pattern of Bieling et al. (2003); however, these results may be explained by different measurements of raising the bar across these studies. 
It was also hypothesized that general eating self-efficacy would significantly predict the general tendency to raise the bar. Results supported this hypothesis and followed the same pattern as clinical perfectionism and ECP, such that general eating self-efficacy significantly predicted the GGSQ total and StanD scores, but not the GGSQ StanI scores. In a 35-year review on goal-setting, Locke and Latham (2002) found that individuals with higher self-efficacy set more challenging goals, were more committed to their goals, used strategies that led to greater goal completion, and responded more positively to negative feedback than individuals with lower self-efficacy. In the context of the present study, Locke and Latham's (2002) findings suggest that increasing self-efficacy may explain why individuals reappraise goals after success. For example, an individual may have just enough perceived self-efficacy to attempt to lose $1 \mathrm{lb}$. of weight per week. Before meeting this goal, $1 \mathrm{lb}$. per week is appraised as sufficient, because it corresponds to his or her level of self-efficacy. Successfully losing $1 \mathrm{lb}$. per week may bolster self-efficacy, such that the previous goal no longer corresponds with the individual's level of self-efficacy. Only at this point does the previous goal seem insufficient. From this perspective, reappraisal is dependent upon increasing self-efficacy rather than dissatisfaction or insufficiency. This perspective could explain the significant association between general eating self-efficacy and greater GGSQ StanD scores. However, it should be noted that the general eating selfefficacy measure used in the present study refers specifically to the ability to control oneself from overeating, whereas the general tendency to raise the bar can refer to goal-setting in any domain. As such, the correlation between these two measures may only be meaningful if participants were focusing on general goal-setting tendencies related to eating behaviours and weight loss. 
4. Raising the bar using weight-related goals. It was hypothesized that higher levels of clinical perfectionism and goal-specific self-efficacy would significantly predict raising the bar in four weight-related goals (i.e., WLG, CCG, EG, and HHG) as demonstrated by a greater increase in goals from Day 1 to Day 8, and from Day 8 to Day 15. These hypotheses were not supported. The only significant predictor to lead to increased change in goals was goal-specific perceived success, where higher perceived success led to raising the bar in CCG from Day 8 to Day 15 for both clinical perfectionism and ECP. CCG was measured by having participants select the number of days they wanted to be at or below the suggested MFP daily calorie limit. Therefore, findings show that when perfectionism and self-efficacy are held constant at the mean, an individual would need to experience a four point increase above the mean in perceived success of the previous week's CCG to predict raising the bar by one calorie day. Although statistically significant, this increase in CCG from Day 8 to Day 15 may not represent a large meaningful change. On average, participants selected just under six out of seven days of the week to follow their MFP calorie consumption daily limit for each goal-setting time point (i.e., Day 1, Day 8, Day 15; see Table 3). Therefore, choosing goals in the upper limit of the available range of goal options from the beginning of the study may have resulted in a ceiling effect that prevented raising the bar in subsequent weeks. This limitation would also hold true for WLG, which restricted goal selection to four possible options ranging between $0.5 \mathrm{lbs}$. and $2 \mathrm{lbs}$. per week. On average, participants selected $1.5 \mathrm{lbs}$. out of a possible $2 \mathrm{lbs}$. to lose per week for each goal-setting time point, once again preventing a large upward shift in weight loss goals from week to week. Indeed, examination of the distribution for CCG and WLG confirmed a strong negative skew (Results not shown). 
However, if the lack of findings is due to a ceiling effect in CCG and WLG, one would expect to see a more consistent pattern of raising the bar in EG and HHG across weeks, which did not impose such strict upper limits. EG had no upper limit in total possible weekly minutes. Examination of the average, minimum, and maximum scores of participants who completed the study (Table 3) reveals a much larger range of goal-setting in this category. HHG, like EG, did not appear limited by a ceiling effect. On average, participants selected approximately six goals to follow per week (out of at least 15 if participants selected not to identify additional goals; Table 3), leaving more opportunity to raise the bar. Additionally, examination of the distribution of EG and HHG suggests that participants selected goals on the lower range of possible goal choices on Day 1, leaving room to raise the bar in subsequent weeks. The impact of HHG was interpreted by examining significant two-way interactions between both perfectionism and success and perfectionism and self-efficacy.

Specifically, it was hypothesized that goal-specific perceived success and self-efficacy would moderate the relationship between perfectionism and raising the bar in weight-related goals, such that those higher in clinical perfectionism would raise the bar when goal-specific perceived success and self-efficacy were high. Although each of these variables interacted with perfectionism independently, moderation was not consistent across time points, weight-related goals, measures of perfectionism, or level of the moderator. However, a few select findings are of note.

Although HHG perceived success moderated the influence of perfectionism on HHG, its influence on perfectionism was contrary to the study's prediction. High levels of HHG perceived success had no influence on change in HHG for either week or for any measure of perfectionism. In contrast, low HHG perceived success moderated the relationship between ECP and raising the 
bar only during Week 1 . The lack of consistent findings across measures of perfectionism or time points make it difficult to interpret these results.

HHG self-efficacy also moderated the relationship between perfectionism and raising the bar. For Week 1, when HHG self-efficacy was low, raising the bar in HHG decreased as ECP increased. The opposite pattern was found when HHG self-efficacy was high, such that raising the bar in HHG increased as perfectionism (i.e., clinical perfectionism and ECP) increased. These results may suggest that raising the bar is dependent on both self-efficacy and perfectionism. Only when self-efficacy is high do perfectionistic individuals raise the bar. The concern about the consequences of making mistakes, being negatively evaluated, and having doubts about actions characteristic of clinical perfectionism and ECP may undermine the ability to set higher goals when self-efficacy is low. In other words, believing in one's own ability acts as a buffer to these negative perfectionistic attributes.

Interestingly, the direction of slopes for high and low HHG self-efficacy was reversed for Week 2. When HHG self-efficacy was low, raising the bar in HHG increased as PSP increased. In contrast, when HHG self-efficacy was high, raising the bar in HHG decreased as clinical perfectionism and ECP increased. It may be that while self-efficacy and perfectionism interacted to predict greater raising the bar during Week 1, individuals were unable to follow through with these goals and therefore lowered the bar to a more realistic level for Week 2. However, these conjectures must be considered cautiously given the inconsistent findings across measures and time points.

In the current study, raising the bar was operationalized as an increase in goal difficulty from one time point to another (e.g., an individual raised the bar if more exercise minutes were selected on Day 15 than on Day 8). However, findings did not show a consistent pattern of goal 
increase across weight-related goals. There are two important considerations that may explain the lack of evidence for raising the bar as measured in the current study. First, it is possible that clinical perfectionists begin by setting the most challenging goal, leaving no room for the possibility of raising the bar. Although clinical perfectionism was not significantly positively correlated with Day 1 weight-related goals, examination of WLG and CCG distributions suggest that participants selected difficult goals from the beginning. Clinical perfectionists are thought to rigidly pursue personally demanding standards even in the midst of adverse consequences (Shafran et al., 2002). ECP and PSP are also characterized by this need to set excessively high standards. Therefore, low self-efficacy, limited resources, or past failure may not prevent the perfectionist from starting with and sticking to the most challenging goal. In the present study, individuals selected among the most difficult CCG and WLG goals on Day 1 (6/7 days, $1.5 / 2 \mathrm{lbs}$.), and these high standards did not waver across time. Therefore, raising the bar may only occur as a domain becomes salient and relevant to the individual. However, once perfectionism is fully manifested in that domain, the standards may be kept as high as possible. For example, a child may not develop perfectionistic tendencies at school until the age where he or she begins to receive performance evaluations (e.g., report cards, homework assignments). To capture a steady increase in goals over time, it may be necessary to measure raising the bar in younger samples as perfectionistic tendencies are developing.

Second, increase in goal challenge over time may be an overly inclusive definition of raising the bar. Participants identified a WLG between $0.5 \mathrm{lbs}$. and $2 \mathrm{lbs}$. per week. However, their MFP profile also depended on a personal total weight loss goal (e.g., 15 lbs.). The current study focused solely on the first; however, it may be that the decision to continue losing $2 \mathrm{lbs}$. per week after successfully losing $15 \mathrm{lbs}$. could be considered as raising the bar for the total 
weight loss goal. It was not possible to assess raising the bar in long-term goals given the 2-week duration of the current study. However, just because an individual consistently selects $2 \mathrm{lbs}$. per week does not necessarily mean that he or she is not raising the bar in some other related area.

5. Post hoc analysis. The post hoc analysis examining the predictive ability of total weight loss from perfectionism revealed that higher levels of clinical perfectionism predicted less overall weight loss. The incongruence between higher clinical perfectionism and worse performance can been explained by the role of failure in Shafran et al.'s (2002) model. Failure to meet one's goals is thought to engender negative self-evaluation and self-criticism in individuals high in clinical perfectionism. In order to avoid failure, clinical perfectionists are hypervigilant of failure cues at the expense of success, which remains unnoticed, unappreciated, or reappraised as insufficient. A strong cognitive focus on failure combined with the tendency to set unrealistically high standard may result to impediments in actual performance (e.g., avoidance, procrastination, distraction) and lower the probability of achieving desired outcomes (Shafran et al., 2002). Interestingly, although higher clinical perfectionism predicted less overall weight loss, completed participants still lost, on average, $1 \mathrm{lb}$. per measurement time (i.e., Day 1 to Day 8 to Day 15). Therefore, while completed participants may have been losing weight by engaging in the study, higher levels of clinical perfectionism may have hindered progress resulting in smaller overall gains.

A post hoc qualitative analysis revealed that individuals who report raising the bar following success (i.e., score high on GGSQ StanI) identify a number of different reasons for raising the bar. These reasons included the (1) attitude toward the goal, (2) resources, (3) difficulty level of the goal, (4) success of the self or others on previous goals, and (5) need to improve the self and set more challenging goals. Examination of statements within these 
categories did not explicitly demonstrate any of the elements related to raising the bar in Shafran et al.'s (2002) model such as fleeting satisfaction with success, fear or avoidance of failure, or reappraisal of the standard as insufficient.

For statements regarding attitudes towards the goal, participants scoring high in GGSQ StanD or CPQ reported that their mood, level of satisfaction, motivation, self-efficacy, or level of stress impacted whether they raised the bar. For the most part, statements did not provide enough information to understand the specifics of these variables. For example, participants did not specify what type of mood or level of satisfaction influences raising the bar. One exception to this was a participant who indicated that raising the bar depended on feeling good, which is in contrast to standard dissatisfaction identified in Shafran et al.'s (2002) model.

For statements regarding one's resources, participants scoring high in GGSQ StanD or CPQ reported that raising the bar necessitates the appropriate resources (e.g., time, energy, flexible schedule). Perceived resources are conceptually related to one's overall self-efficacy. Although resources and self-efficacy are not elements referenced in Shafran et al.'s (2002) model, having adequate resources may increase overall self-efficacy, leading to raising the bar.

For statements regarding the difficulty of the goal, participants scoring high in GGSQ StanD or CPQ reported that raising the bar was influenced by whether the goal was easy, difficult, or realistic. Those scoring high in perfectionism are characterized by setting personally demanding, excessive standards. Realistic goal-setting is somewhat inconsistent with this definition. However, it could be that individuals high in perfectionism believe that their goals are realistic, whereas outside observers perceive them as excessive.

For statements regarding one's level of success, participants scoring high in GGSQ StanD or CPQ reported that raising the bar was influenced by the level of previous self- or other- 
success. According to the model, individuals high in perfectionism experience fleeting positive feelings following success, and are more heavily focused on fear of failure than the accomplishment of a goal. While success is clearly a factor in raising the bar, participants' responses do not provide any information on the appraisal of success.

For statements regarding self-improvement, one participant scoring high in GGSQ StanD reported that raising the bar was influenced by the need to obtain better results, and another scoring high in CPQ acknowledged that there is always room for self-improvement. The need to obtain better results might be indicative of GGSQ StanD's lack of satisfaction upon meeting goals. However, fear of failure rather than self-improvement is thought to be the driving force for clinical perfectionism (Shafran et al., 2002). Overall, only two participants scoring high in either GGSQ StanD or the CPQ referenced anything related to the need to set higher standards. Examining self-improvement statements from participants not scoring in the top third of the GGSQ StanD or CPQ, one participant mentioned raising the bar occurs when previous "results were not enough." This statement seems to line up most closely with Shafran et al.'s (2002) idea of standard dissatisfaction.

In summary, participants often did not provide enough information to fully understand the meaning of reasons for raising the bar. However, this qualitative analysis suggests that raising the bar can occur in individuals high in standard dissatisfaction and clinical perfectionism for reasons beyond those identified by Shafran et al. (2002). For example, raising the bar can occur when individuals are motivated or excited about a goal, or when they feel they have the resources to accomplish a goal. Overall, the post hoc prediction that open-ended statements explaining reasons for raising the bar would map onto the conceptual understanding of raising the bar in clinical perfectionists (Shafran et al., 2002) is not strongly supported. 


\section{Implications of Findings and Future Recommendations}

Research on raising the bar has generated inconsistent results. Stoeber et al. (2008) and Kobori et al. (2009) found evidence in support of raising the bar, Lo and Abbott (2013a) found that raising the bar only occurred when expectations were low, and Egan et al. (2012) did not find evidence for raising the bar as predicted by the cognitive-behavioural model of clinical perfectionism. Interpretations of these findings are difficult given the use of different perfectionism measures and procedures across studies. In the current study, it was postulated that the inconsistency of previous findings could be attributed to the fact that raising the bar was not evaluated in standards that are self-imposed, personally demanding, or in a salient life domain. However, hypotheses were not supported even in a sample of participants that wished to engage in goal-setting for weight loss and were able to select individual goals.

Instead, a discrepancy was discovered between the two measures of raising the bar (i.e., GGSQ and weight-related raising the bar). Participants high in perfectionism reported a greater tendency to experience standard dissatisfaction and reappraisal. While setting more challenging goals is theoretically thought to follow from this emotional and cognitive reaction, perfectionism did not predict a stable pattern of raising the bar across four weight-related goals. Traditionally, personality research has assumed, albeit speciously, a one-to-one relationship between personality and behaviour; the stronger a disposition in an individual, the more of that disposition the individual will demonstrate (Mischel \& Shoda, 1995). The underlying assumption in past research on raising the bar is that participants higher in perfectionism should display higher levels of raising the bar, regardless of situation or task.

In personality research, variability in behaviour across situations has been treated as methodological error (Mischel \& Shoda, 1995). The solution to this error has often been to 
measure behavioural demonstrations of the personality trait (e.g., raising the bar in perfectionism) in a number of situations to create an aggregate score of the "true" level of that trait. However, evidence demonstrates considerable cross-situational variability in behaviour, making it very difficult to find a relationship between self-reported personality and aggregate scores in behaviours that demonstrate that personality trait (Mischel, 1968; Mischel \& Shoda, 1995). Mischel (1968) has labeled the disparity between self-report and behavioural personality as the Personality Paradox. Results from the Carlton Behaviour Study (Mischel \& Peake, 1982; Mischel \& Shoda, 1995) revealed that participants with high self-reported cross-situational consistency of conscientiousness did not demonstrate greater actual consistency in behavioural conscientiousness as rated by family and friends than participants with low self-reported crosssectional consistency in conscientiousness. Self-report and behavioural conscientiousness were only consistent when comparing the temporal stability of specific situations (e.g., Time 1 class attendance with Time 2 class attendance; Mischel \& Peake, 1982; Mischel \& Shoda, 1995). Mischel and Shoda (1995) further demonstrated this temporal relationship between personality and behaviour across a number of situations in children at a summer camp. They found that when evaluating level of aggression, individual children showed similar behavioural variation in aggression depending on the situation. For example, the level of aggression demonstrated by a child teased by his peers one week was related to his level of aggression in that situation over time.

Rather than attributing cross-situational variability to error, Mischel $(1973,2009)$ advocates for a constructivist and dynamic view of personality and situation, where an individual's behaviour is dependent on the meaning ascribed to the situation. The same situation will mean different things to individuals with varying dispositions, depending on their individual 
goals, motivations, level of self-efficacy, and expectations. From this perspective, personality predicts behaviour within an "If ... then ..." paradigm, where all of these variables influence personality to determine behaviour (Mischel, 2009). This is consistent with Shafran et al.'s (2002) definition of clinical perfectionism, which postulates that perfectionistic behaviours will only manifest in highly salient domains specific to the individual. Although a clinical perfectionist might self-report elevated fear of failure, setting of high standards, dissatisfaction with success, or tendency to raise the bar across situation (as is done for measures like the CPQ or GGSQ), this will not unidimensionally predict raising the bar across situation. Therefore, inconsistent patterns of raising the bar across weight-related goals as predicted by clinical perfectionism may be due to a constellation of factors specific to each perfectionist. Observational research may be a preliminary step needed to understand the unique situational meaning that contributes to raising the bar. Perhaps a methodology similar to the Carlton or camp studies (Mischel \& Peake, 1982; Mischel \& Shoda, 1995) would permit the identification of behavioural signatures of raising the bar in individuals with clinical perfectionism. This would require identifying a sample of clinical perfectionists and observing them in areas they report as salient and relevant to their perfectionism.

To the researcher's knowledge, Riley and Shafran (2005) are the only authors to qualitatively analyze the maintaining mechanisms of clinical perfectionism. Shafran et al. (2002) posited that if a clinical perfectionist's "standard for performance is met, it is usually viewed as insufficiently personally demanding and is therefore raised" (p. 779). Using grounded theory (Glaser \& Strauss, 1967), Riley and Shafran (2005) provided evidence for standard dissatisfaction following success and subsequent standard reappraisal. Specifically, while individuals with and without the core features of clinical perfectionism reportedly reacted 
positively to success, this positive reaction was more short-lived in individuals with clinical perfectionism. One participant reported that this feeling disappears "within a couple of days, and often even shorter, because you've achieved it you think well, it's not that brilliant anyway" ( $p$. 372). The comment at the end of this statement regarding brilliance may be a demonstration of standard reappraisal. Riley and Shafran (2005) did not examine raising the bar in these individuals.

Shafran et al.'s (2002) model was created to surpass descriptive phenomenology by providing empirical hypotheses that test the maintaining mechanisms of clinical perfectionism. Shafran et al. (2002) provide four possible hypotheses with regards to maintaining mechanisms, one of which is the reappraisal of standards as insufficient upon success. It is interesting to note that the behavioural act of raising the bar is not included among these four mechanisms, and is instead understood as sequelae to the maintaining mechanism of standard dissatisfaction and reappraisal within clinical perfectionism. Furthermore, Shafran et al. (2002) do not specifically call for exploration of raising the bar. As described in the fallacy affirming the consequent (i.e., if A then B, B then A), evidence of raising the bar in clinical perfectionists does not provide support for standard dissatisfaction and reappraisal. Raising the bar can occur for a variety of reasons, as seen in the qualitative analysis of the present study. Therefore, identifying whether raising the bar occurs does not provide the information necessary to validate the maintaining mechanism of standard dissatisfaction and reappraisal, and as such does not provide the most pertinent information needed for clinical assessment and intervention. For example, raising the bar in clinical perfectionism may only be a treatment target if followed by high levels of standard dissatisfaction and reappraisal. Instead, a more likely target of treatment would be the selfcriticism and fear of failure engendered from the negative cognitive and emotional reactions to 
success. Therefore, it is suggested that the focus of future studies be redirected to the examination of standard dissatisfaction and reappraisal as outlined in Shafran et al.'s (2002) hypotheses. If A then B holds true, then raising the bar (B) should only be examined if the standard dissatisfaction and reappraisal (A) has been confirmed. Furthermore, given the crosssituational inconsistency of behaviour as predicted from measures of personality (Mischel, 1968), experimental research is not likely to demonstrate a clear consistent pattern of raising the bar as predicted by measures of perfectionism. Riley and Shafran (2005) used grounded theory to explore maintaining mechanisms of clinical perfectionism. However, they did not provide a lot of evidence for the reappraisal of standards. Therefore, this qualitative methodology may be a good starting point to develop this hypothesis.

\section{Limitations}

The current study has several important limitations. First, the interpretation of findings is limited to raising the bar in eating and weight loss for individuals who are overweight or obese and wish to lose weight. The manifestation of perfectionism will depend completely on whether the individual finds the life domain important, and whether standards are self-imposed and personally demanding. Furthermore, given the cross-situational inconsistency between personality and behaviour, inconsistent findings across individuals of similar levels of clinical perfectionism would likely occur for goals within the same salient life domain (e.g., WLG vs. $\mathrm{EG})$.

Second, the measurement of weight-related goals was limited by a possible ceiling effect (e.g., elevated starting average of WLG and CCG). The current study attempted to prevent this ceiling effect by asking participants if they wanted to select a WLG of greater than $2 \mathrm{lbs}$. at each goal-setting time point. However, the majority of participants did not opt for this option, 
preventing further analysis. It may be necessary to explore raising the bar in goals that have no upper limit. However, if a ceiling effect was influencing results, a more consistent pattern of results would have been expected for EG and HHG as these goals did not impose a ceiling effect.

Third, the current study did not take into account the difficulty level of the combined effect of weight-related goals. While individual goals (e.g., WLG, CCG, EG, and HHG) may have fluctuated from week to week, a decrease in one goal (e.g., HHG) may have occurred given an increase in another goal (i.e. EG). However, given the variety of measurement units used across weight-related goals (e.g., lbs., days, minutes, number selected), the combined effect of weight-related goals would be difficult to quantify and interpret. Moreover, Mischel (1968) has cautioned against attempting to predict cross-situational behaviours (e.g., raising the bar in aggregated weight loss goals) from personality traits such as perfectionism.

Fourth, increase in goals was only measured at two weekly time points using change scores. This cross-sectional analysis prevents a clear interpretation of different patterns across weeks such as the opposing pattern of raising the bar in HHG for Week 1 and Week 2. Change scores also do not allow for the evaluation of long-term raising the bar (e.g., consistently losing 2lbs. per week even after meeting one's total weight loss goal of $15 \mathrm{lbs}$.). Understanding the fluctuation of goals over longer periods of time as predicted by variables such as perfectionism, self-efficacy, and success would allow for better interpretation of raising the bar. Therefore, it may be beneficial to look at the trend of goal-setting longitudinally through the use of growth curve modeling rather than evaluating the influence of perfectionism using change scores.

Fifth, psychometric, procedural, and experimenter artifacts may have influenced the results. With regards to the psychometrics of the CPQ, the present study's Cronbach's $\alpha$ of .63 was lower than the CPQ of other nonclinical samples (e.g., $\alpha=.83$, Chang \& Sanna, 2012; $\alpha=$ 
.71, Egan et al., 2016). As previously mentioned, preliminary studies have suggested that the level of clinical perfectionism can be better captured by removing items from the total scale and dividing the remaining items into separate factors. However, recommendations regarding which items should be retained to create these two factors are inconsistent (Egan et al., 2016; Dickie et al., 2012, Stoeber \& Damien, 2014). Furthermore, internal consistency does not improve by much even when such items are removed (e.g., $\alpha=.79$; Stoeber \& Damian, 2014). The use of the CPQ to measure perfectionism as conceptualized by Shafran et al. (2002) is still in its infancy. More research is needed to further refine the reliability and factor structure of the CPQ. Although a low Cronbach's $\alpha$ limits interpretation of findings in the present study, all analyses were also performed using the ECP, which shares features with the CPQ such as negative self-evaluation based on performance and the need to meet excessively high standards.

With regards to procedure and experimenter artifacts, completing measures of perfectionism prior to beginning the study may have artificially influenced subsequent goalsetting. Likewise, completing the GGSQ immediately before responding to the open-ended question on standard setting may have influenced participant responses. Information about the benefits of specific goal-setting and consistent self-monitoring may have led participants to set more challenging goals and to report higher levels of success and self-efficacy than they might have when completing these tasks alone. The influence of demand characteristics were demonstrated by a select number of participants who gained weight throughout the study. These participants shared concern that their lack of success would negatively influence study results, which suggests that motivation to succeed was influenced by the fact that weight loss was occurring in the context of a psychological study. These artifacts were hopefully reduced by allowing participants to complete the GSQ alone in the experimentation room. However, it 
would have been beneficial to have participants complete perfectionism and GGSQ questionnaires at the end of the study prior to debrief, or at a separate time several weeks prior or following the study.

Sixth, the current study did not collect data regarding the motivation behind goal-setting or raising the bar. As demonstrated by the qualitative analysis, a number of reasons can explain why an individual set a particular goal or raised the bar (e.g., attitude, resources, difficulty, success, self-improvement). Although participants identified motivations for raising the bar in general, these motivations do not necessarily translate to specific weight-related goal-setting. Given that Shafran et al. (2002) suggest that raising the bar is most important when it occurs in response to standard dissatisfaction and reappraisal, future studies should analyze the motivation behind standard setting and raising the bar.

Finally, nonsignificant results were likely influenced by a lack of statistical power (1- $\beta$ ) to predict raising the bar from clinical perfectionism. A priori power analysis was complicated to conduct for the current study as previous research on raising the bar has employed different procedures, measures of perfectionism, and statistical analyses than the present study. Egan et al.'s (2012) methods and procedure aligned most closely with the current study. Both used the CPQ to predict change scores at two time points and evaluated raising the bar using moderated hierarchical regression. However, in spite of these similarities, Egan et al. (2012) do not provide an adequate estimate of the effect of CPQ on raising the bar given (1) the inclusion of additional predictors into their model not used in the current study (i.e., positive and negative affect), and (2) the lack of a significant effect of CPQ or its interaction with success on raising the bar. As such, using the effect of Egan et al.'s (2012) study does not provide an accurate interpretation of the effect of CPQ on raising the bar, but rather of the combined effect of nonsignificant CPQ and 
other significant variables on raising the bar. Some researchers have recommended calculating a post hoc power analysis to determine whether the nonsignificant result is due to low power rather than lack of effect; however, retrospective power calculations do not provide any additional information that the $p$-value or CIs have not already provided (Hoenig \& Heisey, 2001; Lenth, 2007a, b). Furthermore, given the direct relationship between the study's $p$-value and the calculated post hoc power, when a nonsignificant result is found, power will always be low (Hoenig \& Heisey, 2001; Lenth 2007b). This leads to the erroneous conclusion that a bigger effect would be found if the study was replicated using a larger sample. Given the lack of an appropriate effect size from previous research and the limitations of conducting a post hoc power analysis, a power analysis was conducted using a hypothetical small effect size. If it is assumed that the effect of CPQ on raising the bar is $f^{2}=.02$ (Cohen, 1992), a G*Power 2 calculation for linear multiple regression: fixed model, $R^{2}$ deviation from zero identifies that a sample of 395 would be necessary to achieve adequate power $(1-\beta=.80)$, at $p=0.05$. This analysis would simply allow for an evaluation of the predictive power of CPQ on raising the bar, but would not allow for two- or three-way interactions. Even Egan et al. (2012), who conducted their study online, were unable to achieve this sample size. The sample size would greatly decrease $(N=55)$ if a medium effect size was used. However, empirical findings are not yet adequate to hypothesize the presence of a medium effect. As such, the major limitation of the current study and previous research on raising the bar is inadequate power.

In spite of these limitations, the current research makes an important contribution to the literature. Qualitative results demonstrate that raising the bar can occur for a number of reasons even when clinical perfectionism is high. Therefore, raising the bar is not specific to clinical perfectionism, nor does it necessarily provide evidence for standard dissatisfaction and 
reappraisal. The present study provides the literature with a new scale for measuring the general tendency to experience standard dissatisfaction and reappraisal and to raise the bar. While clinical perfectionism significantly predicts the emotional and cognitive reaction of dissatisfaction to success, it does not provide evidence for the behavioural follow-through of raising the bar in the domain of eating and weight loss. These results are consistent with the personality paradox (Mischel, 1968), where self-reported personality traits do not necessarily align with predicted behavioural manifestations of these traits in specific situations. Finally, this study calls for a redirection of attention back to the fundamental hypotheses of Shafran et al. (2002), with specific attention to the reappraisal of standards as insufficient after success. 
Table 1

Differences in Measures Between Completers and Noncompleters

\begin{tabular}{|c|c|c|c|c|}
\hline \multirow[b]{2}{*}{ Measure } & \multicolumn{2}{|c|}{$\operatorname{Mean}(S D)$} & \multirow[b]{2}{*}{$t(\mathrm{df})$} & \multirow[b]{2}{*}{$p$} \\
\hline & Completers $(n=66)$ & Noncompleters $(n=20)$ & & \\
\hline CPQ & $28.29(4.64)$ & $25.58(5.24)$ & $-2.22(84)$ & .029 \\
\hline PSP & $0.34(1.73)$ & $-1.12(2.00)$ & $-3.19(84)$ & .002 \\
\hline EDEQ_R & $2.57(1.61)$ & $1.72(1.28)$ & $-2.15(84)$ & .034 \\
\hline DASS_S & $35.12(11.57)$ & $27.26(8.14)$ & $-3.40(44.51)$ & .001 \\
\hline
\end{tabular}

Note. $\mathrm{CPQ}=$ Clinical Perfectionism Questionnaire; $\mathrm{PSP}=$ Personal standards perfectionism; EDEQ_R = Eating Disorders Evaluation Questionnaire, Dietary Restraint subscale; DASS_S = Depression Anxiety Stress Scales_21, Stress subscale. 
Table 2

Comparison of Mean Score to Total Possible Score on Primary Variables

\begin{tabular}{lcc}
\hline Measure & $\begin{array}{c}\text { Total } \\
\text { Possible }\end{array}$ & Mean $(S D)$ \\
\hline GGSQ Total & 72 & $35.86(8.26)$ \\
GGSQ StanD & 42 & $20.23(7.59)$ \\
GGSQ StanI & 21 & $15.64(3.24)$ \\
CPQ & 48 & $28.29(4.64)$ \\
ECP & $z$-score & $0.15(2.58)$ \\
PSP & $z$-score & $0.34(2.57)$ \\
DASS_A & 42 & $26.55(10.33)$ \\
DASS_S & 42 & $35.12(11.57)$ \\
DASS_D & 42 & $30.00(12.89)$ \\
ESES & 175 & $94.26(32.69)$ \\
EDEQ Total & 6 & $3.42(1.32)$
\end{tabular}

Note. Mean and SD based on completed participants $(N=66)$. GGSQ $=$ General Goal-setting Questionnaire; Total $=$ total score; $\mathrm{StanD}=$ Standard Dissatisfaction subscale; StanI $=$ Standard Increase subscale; $\mathrm{CPQ}=C$ linical Perfectionism Questionnaire; ECP = Evaluative concerns perfectionism; PSP = Personal standards perfectionism; DASS = Depression Anxiety Stress Scales_21; A = Anxiety subscale; S = Stress subscale; D = Depression subscale; ESES = Eating Self-Efficacy Scale $; \mathrm{EDEQ}=$ Eating Disorders Examination Questionnaire . 
Table 3

Descriptive Statistics for Weight-Related Goals

\begin{tabular}{lcccc}
\hline Measure & & & & \\
\hline WLG & Mean $(S D)$ & Min. & Max. & Mode \\
\hline D1 & $1.59(0.46)$ & 0.5 & 2 & 2 \\
D8 & $1.55(0.47)$ & 0.5 & 2 & 2 \\
D15 & $1.46(0.52)$ & 0.5 & 2 & 2 \\
\hline CCG & & & & \\
\hline D1 & $5.61(1.68)$ & 1 & 7 & 7 \\
D8 & $5.78(1.35)$ & 2 & 7 & 7 \\
D15 & $5.58(1.46)$ & 1 & 7 & 7 \\
\hline EG & & & & \\
\hline D1 & $201.53(142.49)$ & 60 & 900 & 180 \\
D8 & $187.61(99.42)$ & 0 & 540 & 180 \\
D15 & $171.40(102.90)$ & 0 & 525 & 90,180 \\
\hline HHG & & & & \\
\hline D1 & $6.28(2.92)$ & 1 & 14 & 5 \\
D8 & $5.92(2.99)$ & 1 & 13 & 4 \\
D15 & $5.27(2.67 * *$ & 1 & 13 & 3 \\
\hline
\end{tabular}

Note. ${ }^{*} \mathrm{HHG}$ Day 15 significantly less than Day 1 or Day $8 . \mathrm{WLG}=$ Weight Loss Goals; $\mathrm{CCG}=$ Calorie

Consumption Goals; EG = Exercise Goals; HHG = Healthy Habit Goals; D1 = Day 1, D8 = Day 8, D15 = Day 15. 
Table 4

Correlation Matrix for GGSQ, Perfectionism, Self-Efficacy and Psychopathology Measures

\begin{tabular}{|c|c|c|c|c|c|c|c|}
\hline Measure & 1 & 2 & 3 & 4 & 5 & 6 & 7 \\
\hline 1. GGSQ Total & & & & & & & \\
\hline 2. GGSQ StanD & $.9 \overline{2 * * *}$ & & & & & & \\
\hline 3. GGSQ StanI & $.40 * *$ & $\overline{.01}$ & & & & & \\
\hline 4. CPQ Total & $.31 *$ & $.25 *$ & $\overline{.21}$ & & & & \\
\hline 5. ECP & $.40^{* *}$ & $.41 * *$ & .06 & $.7 \overline{1 * * *}$ & & & \\
\hline 6. PSP & .10 & .02 & .20 & $.51 * * *$ &.$\overline{41 * *}$ & & \\
\hline 7. ESES Total & $.27 *$ & $.32 * *$ & -.06 & $.37 * *$ & $.54 * * *$ & .16 & \\
\hline DASS_A & $.45 * * *$ & $.47 * * *$ & .04 & $.58 * * *$ & $.74 * * *$ & $.29 *$ & $.56^{* * *}$ \\
\hline DASS_S & $.38 * *$ & $.36 * *$ & .06 & $.66 * * *$ & $.72 * * *$ & $.37 * *$ & $.57 * * *$ \\
\hline DASS_D & $.37 * *$ & $.41 * *$ & .03 & $.46^{* * *}$ & $.67 * * *$ & .18 & $.49 * * *$ \\
\hline EDEQ Total & $.21^{\mathrm{t}}$ & $.23^{\mathrm{t}}$ & .01 & $.46^{* * *}$ & $.58 * * *$ & $.25^{*}$ & $.47 * * *$ \\
\hline
\end{tabular}

Note. 1 participant completed the GGSQ following debrief and was therefore removed from analyses. $N=65$ for correlations with GGSQ. GGSQ = General Goal-setting Questionnaire; Total = total score, StanD = Standard Dissatisfaction subscale, $\mathrm{StanI}=$ Standard Increase subscale; $\mathrm{CPQ}=$ Clinical Perfectionism Questionnaire; $\mathrm{ECP}=$ Evaluative concerns perfectionism; PSP = Personal standards perfectionism; ESES = Eating Self-Efficacy Scale; DASS $=$ Depression Anxiety Stress Scales_21; A = Anxiety subscale; $\mathrm{S}=$ Stress subscale; $\mathrm{D}=$ Depression subscale. $\mathrm{EDEQ}=$ Eating Disorders Examination Questionnaire.

${ }^{\mathrm{t}} .05<\mathrm{p}>.10, * \mathrm{p}<.05 . * * \mathrm{p}<.01 ., * * * \mathrm{p}<.001$ 
Table 5

Linear Regression for Predicting the General Tendency to Raise the Bar (GGSQ).

\begin{tabular}{|c|c|c|c|c|c|c|}
\hline \multirow{3}{*}{ Predictor } & \multicolumn{6}{|c|}{ GGSQ } \\
\hline & \multicolumn{2}{|c|}{ Total } & \multicolumn{2}{|c|}{ StanD } & \multicolumn{2}{|c|}{ StanI } \\
\hline & $R^{2}$ & $\beta$ & $R^{2}$ & $\beta$ & $R^{2}$ & $\beta$ \\
\hline CPQ & 0.097 & $0.549 *$ & 0.063 & $.406 *$ & NS & $\mathrm{NS}$ \\
\hline ECP & 0.158 & $1.266^{* *}$ & 0.165 & $1.182 * *$ & NS & NS \\
\hline PSP & NS & NS & NS & NS & NS & NS \\
\hline ESES & 0.072 & $0.068 *$ & 0.103 & $.074 * *$ & NS & NS \\
\hline
\end{tabular}

Note. $\mathrm{N}=65$, as 1 participant completed the GGSQ following debrief and was therefore removed from analyses.

Each predictor was entered into a separate linear regression for the GGSQ total and factor scores, creating a total of 12 linear regressions. GGSQ = General Goal-setting Questionnaire; Total = total Score; StanD = Standard

Dissatisfaction subscale; $\mathrm{StanI}=$ Standard Increase subscale; $\mathrm{CPQ}=$ Clinical Perfectionism Questionnaire $; \mathrm{ECP}=$ Evaluative concerns perfectionism; PSP = Personal standards perfectionism; ESES = Eating Self-Efficacy Scale. NS $=$ Nonsignificant.

$* p<.05, * * p<.01$ 
Table 6

Hierarchical Multiple Regression Analyses Predicting Week 1 Change in Weight-Related Goals From CPQ

\begin{tabular}{|c|c|c|c|c|c|c|c|c|}
\hline \multirow[b]{3}{*}{ Predictor } & \multicolumn{8}{|c|}{ Week 1 Change in GSQ Goal Domain } \\
\hline & \multicolumn{2}{|c|}{ WLG } & \multicolumn{2}{|c|}{$\mathrm{CCG}$} & \multicolumn{2}{|c|}{ EG } & \multicolumn{2}{|c|}{ HHG } \\
\hline & $\Delta R^{2}$ & $\beta$ & $\Delta R^{2}$ & $\beta$ & $\Delta R^{2}$ & $\beta$ & $\Delta R^{2}$ & $\beta$ \\
\hline Step 1 & $.22 * *$ & & NS & & NS & & .03 & \\
\hline CPQ & & $\begin{array}{c}.01 \\
(-0.02-0.03)\end{array}$ & & & & & & $\begin{array}{c}.08 \\
(-0.04-0.19)\end{array}$ \\
\hline $\mathrm{SE}$ & & $\begin{array}{c}.02 \\
(-0.07-0.12)\end{array}$ & & & & & & $\begin{array}{c}-.03 \\
(-0.84-0.84)\end{array}$ \\
\hline Success & & $\begin{array}{c}.08 * * \\
(0.04-0.12)\end{array}$ & & & & & & $\begin{array}{c}.05 \\
(-0.35-0.41)\end{array}$ \\
\hline Step 2 & .06 & & NS & & NS & & $.10^{\mathrm{t}}$ & \\
\hline CPQ x SE & & $\begin{array}{c}.03^{\mathrm{t}} \\
(.00-.06)\end{array}$ & & & & & & $\begin{array}{c}.23 * \\
(-0.03-0.57)\end{array}$ \\
\hline CPQ $\mathrm{x}$ & & -.00 & & & & & & -.05 \\
\hline Success & & $(-.02-.01)$ & & & & & & $(-0.16-0.07)$ \\
\hline SE $x$ & & .00 & & & & & & .20 \\
\hline Success & & $(-.03-.04)$ & & & & & & $(-0.29-0.50)$ \\
\hline Step 3 & NS & & NS & & NS & & $.04^{\mathrm{t}}$ & \\
\hline $\begin{array}{c}\text { CPQ x SE x } \\
\text { Success }\end{array}$ & & & & & & & & $\begin{array}{c}.06 \\
(-0.8-0.12)\end{array}$ \\
\hline
\end{tabular}

Note. All predictor variables are centered on the variable mean. $\Delta R^{2}$ and $\beta$ based on 1000 bootstrap samples to control for violations in normality of errors and homoscedasticity. Bias corrected and accelerated (BCa) $95 \%$ confidence intervals (CI) provided in parentheses. Week 1 represents the change between goals set on Day 1 and goals set on Day 8. WLG = Weight Loss Goal; CCG = Calorie Consumption Goal; EG = Exercise Goal; HHG = Healthy Habit Goal; CPQ = Clinical

Perfectionism Questionnaire; $\mathrm{SE}=$ Goal-specific self-efficacy; Success = Goal-specific perceived success; NS = Non significant

t. $05<\mathrm{p}>.10, * \mathrm{p}<.05 . * * \mathrm{p}<.01 ., * * * \mathrm{p}<.001$ 
Table 7

Hierarchical Multiple Regression Analyses Predicting Week 1 Change in Weight-Related Goals From ECP

\begin{tabular}{|c|c|c|c|c|c|c|c|c|}
\hline \multirow[b]{3}{*}{ Predictor } & \multicolumn{8}{|c|}{ Week 1 Change in GGSQ Goal Domain } \\
\hline & \multicolumn{2}{|c|}{ WLG } & \multicolumn{2}{|c|}{ CCG } & \multicolumn{2}{|c|}{ EG } & \multicolumn{2}{|c|}{$\mathrm{HHG}$} \\
\hline & $\Delta R^{2}$ & $\beta$ & $\Delta R^{2}$ & $\beta$ & $\Delta R^{2}$ & $\beta$ & $\Delta R^{2}$ & $\beta$ \\
\hline Step 1 & $.24 * *$ & & NS & & NS & & -.03 & \\
\hline $\mathrm{ECP}$ & & .02 & & & & & & .11 \\
\hline & & $(-0.01-0.07)$ & & & & & & $(-0.12-0.35)$ \\
\hline SE & & .03 & & & & & & -.06 \\
\hline & & $(-0.07-0.12)$ & & & & & & $(-0.74-0.72)$ \\
\hline Success & & $.08 * *$ & & & & & & .04 \\
\hline & & $(0.04-0.12)$ & & & & & & $(-0.38-0.53)$ \\
\hline Step 2 & .06 & & NS & & NS & & $.15^{*}$ & \\
\hline $\mathrm{ECP} \times \mathrm{SE}$ & & $.04^{\mathrm{t}}$ & & & & & & $.53 * *$ \\
\hline & & $(-0.00-0.10)$ & & & & & & $(0.16-0.83)$ \\
\hline ECP $x$ & & .00 & & & & & & $-.22 *$ \\
\hline Success & & $(-0.01-0.02)$ & & & & & & $(-0.47-0.04)$ \\
\hline SE $x$ & & -.00 & & & & & & .18 \\
\hline Success & & $(-0.04-0.03)$ & & & & & & $(-0.26-0.44)$ \\
\hline Step 3 & NS & & NS & & NS & & & \\
\hline ECP x SE x & & & & & & & NS & \\
\hline Success & & & & & & & & \\
\hline
\end{tabular}

Note. All predictor variables are centered on the variable mean. $\Delta R^{2}$ and $\beta$ based on 1000 bootstrap samples to control for violations in normality of errors and homoscedasticity. Bias corrected and accelerated (BCa) $95 \%$ confidence intervals (CI) provided in parentheses. Week 1 represents the change between goals set on Day 1 and goals set on Day 8. WLG = Weight Loss Goal; CCG = Calorie Consumption Goal; EG = Exercise Goal; HHG = Healthy Habit Goal; ECP = Evaluative concerns perfectionism; $\mathrm{SE}=$ Goal-specific self-efficacy; Success $=$ Goal-specific perceived success; NS = Nonsignificant.

${ }^{\mathrm{t}} .05<\mathrm{p}>.10, * \mathrm{p}<.05$. ** $\mathrm{p}<.01 ., * * * \mathrm{p}<.001$ 
Table 8

Hierarchical Multiple Regression Analyses Predicting Week 1 Change in Weight-Related Goals From PSP

\begin{tabular}{|c|c|c|c|c|c|c|c|c|}
\hline \multirow[b]{3}{*}{ Predictor } & \multicolumn{8}{|c|}{ Week 1 Change in GGSQ Goal Domain } \\
\hline & \multicolumn{2}{|c|}{ WLG } & \multicolumn{2}{|c|}{$\mathrm{CCG}$} & \multicolumn{2}{|c|}{ EG } & \multicolumn{2}{|c|}{ HHG } \\
\hline & $\Delta R^{2}$ & $\beta$ & $\Delta R^{2}$ & $\beta$ & $\Delta R^{2}$ & $\beta$ & $\Delta R^{2}$ & $\beta$ \\
\hline Step 1 & $.21 * *$ & & NS & & NS & & NS & \\
\hline PSP & & .04 & & & & & & \\
\hline & & $(-0.02-0.11)$ & & & & & & \\
\hline SE & & .01 & & & & & & \\
\hline & & $(-0.09-0.10)$ & & & & & & \\
\hline Success & & $.09 * *$ & & & & & & \\
\hline & & $(0.04-0.13)$ & & & & & & \\
\hline Step 2 & .05 & & NS & & NS & & NS & \\
\hline PSP $x$ SE & & $0.06^{\mathrm{t}}$ & & & & & & \\
\hline & & $(-0.02-0.12)$ & & & & & & \\
\hline PSP $x$ & & -0.01 & & & & & & \\
\hline Success & & $(-0.04-0.03)$ & & & & & & \\
\hline SE $x$ & & 0.01 & & & & & & \\
\hline Success & & $(-0.03-0.03)$ & & & & & & \\
\hline Step 3 & NS & & NS & & NS & & NS & \\
\hline PSP $x$ SE $x$ & & & & & & & & \\
\hline Success & & & & & & & & \\
\hline
\end{tabular}

Note. All predictor variables are centered on the variable mean. $\Delta R^{2}$ and $\beta$ based on 1000 bootstrap samples to control for violations in normality of errors and homoscedasticity. Bias corrected and accelerated (BCa) 95\% confidence intervals (CI) provided in parentheses. Week 1 represents the change between goals set on Day 1 and goals set on Day 8. WLG = Weight Loss Goal; CCG = Calorie Consumption Goal; EG = Exercise Goal; HHG = Healthy Habit Goal; PSP = Personal standards perfectionism; SE = Goal-specific self-efficacy; Success = Goal-specific perceived success; NS = Nonsignificant.

t. $05<\mathrm{p}>.10, * \mathrm{p}<.05 . * * \mathrm{p}<.01 ., * * * \mathrm{p}<.001$ 
Table 9

Hierarchical Multiple Regression Analyses Predicting Week 2 Change in Weight-Related Goals From CPQ

\begin{tabular}{|c|c|c|c|c|c|c|c|c|}
\hline \multirow[b]{3}{*}{ Predictor } & \multicolumn{8}{|c|}{ Week 2 Change in GGSQ Goal Domain } \\
\hline & \multicolumn{2}{|c|}{ WLG } & \multicolumn{2}{|c|}{ CCG } & \multicolumn{2}{|c|}{ EG } & \multicolumn{2}{|c|}{$\mathrm{HHG}$} \\
\hline & $\Delta R^{2}$ & $\beta$ & $\Delta R^{2}$ & $\beta$ & $\Delta R^{2}$ & $\beta$ & $\Delta R^{2}$ & $\beta$ \\
\hline Step 1 & NS & & $.06^{t}$ & & $.13 *$ & & .07 & \\
\hline CPQ & & & & $\begin{array}{c}.03 \\
(-0.05-.12)\end{array}$ & & $\begin{array}{c}2.21 \\
(0.07-4.96)\end{array}$ & & $\begin{array}{c}-.04 \\
(-0.12-0.06)\end{array}$ \\
\hline $\mathrm{SE}$ & & & & $\begin{array}{c}-.31 \\
(-0.73-0.17)\end{array}$ & & $\begin{array}{c}-9.29 \\
(-27.01-8.65)\end{array}$ & & $\begin{array}{c}-.37 \\
(-1.06-0.35)\end{array}$ \\
\hline Success & & & & $\begin{array}{c}.28 * * \\
(0.10-0.47)\end{array}$ & & $\begin{array}{c}9.42^{\mathrm{t}} \\
(-0.22-19.43)\end{array}$ & & $\begin{array}{c}.33 \\
(-0.12-0.78)\end{array}$ \\
\hline $\begin{array}{l}\text { Step } 2 \\
\quad \text { CPQ x SE }\end{array}$ & NS & & NS & & NS & & $.12 *$ & $\begin{array}{c}-.14^{* *} \\
(-0.29--0.05)\end{array}$ \\
\hline CPQ $x$ & & & & & & & & $.06^{\mathrm{t}}$ \\
\hline Success & & & & & & & & $(-0.04-0.18)$ \\
\hline SE $x$ & & & & & & & & $.18^{\mathrm{t}}$ \\
\hline Success & & & & & & & & $(-0.24-0.67)$ \\
\hline $\begin{array}{l}\text { Step } 3 \\
\text { CPQ x SE x } \\
\text { Success }\end{array}$ & NS & & NS & & NS & & NS & \\
\hline
\end{tabular}

Note. All predictor variables are centered on the variable mean. $\Delta R^{2}$ and $\beta$ based on 1000 bootstrap samples to control for violations in normality of errors and homoscedasticity. Bias corrected and accelerated (BCa) 95\% confidence intervals (CI) provided in parentheses. Week 2 represents the change between goals set on Day 8 and goals set on Day 15 . WLG = Weight Loss Goal; $\mathrm{CCG}=$ Calorie Consumption Goal; EG = Exercise Goal; HHG = Healthy Habit Goal; CPQ = Clinical Perfectionism Questionnaire; SE = Goal-specific Self-efficacy; Success = Goal-specific perceived success; NS = Nonsignificant.

t. $05<\mathrm{p}>.10, * \mathrm{p}<.05 . * * \mathrm{p}<.01 ., * * * \mathrm{p}<.001$ 
Table 10

Hierarchical Multiple Regression Analyses Predicting Week 2 Change in Weight-Related Goals From ECP

\begin{tabular}{|c|c|c|c|c|c|c|c|c|}
\hline \multirow[b]{3}{*}{ Predictor } & \multicolumn{8}{|c|}{ Week 2 Change in GGSQ Goal Domain } \\
\hline & \multicolumn{2}{|c|}{ WLG } & \multicolumn{2}{|c|}{ CCG } & \multicolumn{2}{|r|}{ EG } & \multicolumn{2}{|r|}{ HHG } \\
\hline & $\Delta R^{2}$ & $\beta$ & $\Delta R^{2}$ & $\beta$ & $\Delta R^{2}$ & $\beta$ & $\Delta R^{2}$ & $\beta$ \\
\hline Step 1 & .05 & & $.10^{t}$ & & $.06^{\mathrm{t}}$ & & .07 & \\
\hline ECP & & .00 & & -.03 & & 2.23 & & -.50 \\
\hline & & $(-0.05-0.05)$ & & $(-0.18-0.12)$ & & $(-2.58-7.39)$ & & $(-0.23-0.12)$ \\
\hline SE & & -.01 & & -.32 & & -9.98 & & -.38 \\
\hline & & $(-0.08-0.10)$ & & $(-0.78-0.13)$ & & $(-28.81-7.83)$ & & $(-1.07-0.29)$ \\
\hline Success & & .04 & & $.25^{*}$ & & $9.97^{\mathrm{t}}$ & & .33 \\
\hline & & $(-0.01-0.07)$ & & $(0.09-0.40)$ & & $(0.08-20.04)$ & & $(-0.03-0.82)$ \\
\hline Step 2 & .04 & & NS & & NS & & $.11^{\mathrm{t}}$ & \\
\hline $\mathrm{ECP} \times \mathrm{SE}$ & & .01 & & & & & & $-.26^{*}$ \\
\hline & & $(-0.05-0.09)$ & & & & & & $(-0.54--0.06)$ \\
\hline ECP $x$ & & -.01 & & & & & & $.11^{\mathrm{t}}$ \\
\hline Success & & $(-0.04-0.01)$ & & & & & & $(-0.03-0.27)$ \\
\hline SE $x$ & & -.01 & & & & & & .19 \\
\hline Success & & $(-0.06-0.05)$ & & & & & & $(-0.30-0.64)$ \\
\hline Step 3 & $.07 *$ & & NS & & NS & & NS & \\
\hline ECP x SE $x$ & & .02 & & & & & & \\
\hline Success & & $(-0.00-0.05)$ & & & & & & \\
\hline
\end{tabular}

Note. All predictor variables are centered on the variable mean. $\Delta R^{2}$ and $\beta$ based on 1000 bootstrap samples to control for violations in normality of errors and

homoscedasticity. Bias corrected and accelerated (BCa) $95 \%$ confidence intervals (CI) provided in parentheses. Week 2 represents the change between goals set on Day 8 and goals set on Day 15. WLG = Weight Loss Goal; $\mathrm{CCG}=$ Calorie Consumption Goal; $\mathrm{EG}=$ Exercise Goal; HHG = Healthy Habit Goal; ECP = Evaluative concerns perfectionism; $\mathrm{SE}=$ Goal-specific Self-efficacy, Success $=$ Goal-specific perceived success; NS = Nonsignificant.

t. $05<\mathrm{p}>.10, * \mathrm{p}<.05 . * * \mathrm{p}<.01 ., * * * \mathrm{p}<.001$ 
Table 11

Hierarchical Multiple Regression Analyses Predicting Week 2 Change in Weight-Related Goals From PSP

\begin{tabular}{|c|c|c|c|c|c|c|c|c|}
\hline \multirow[b]{3}{*}{ Predictor } & \multicolumn{8}{|c|}{ Week 2 Change in GGSQ Goal Domain } \\
\hline & \multicolumn{2}{|c|}{ WLG } & \multicolumn{2}{|c|}{ CCG } & \multicolumn{2}{|r|}{ EG } & \multicolumn{2}{|c|}{ HHG } \\
\hline & $\Delta R^{2}$ & $\beta$ & $\Delta R^{2}$ & $\beta$ & $\Delta R^{2}$ & $\beta$ & $\Delta R^{2}$ & $\beta$ \\
\hline Step 1 & NS & & $.10^{\mathrm{t}}$ & & $.12 *$ & & $.11^{\mathrm{t}}$ & \\
\hline PSP & & & & -.06 & & 5.47 & & $.22^{\mathrm{t}}$ \\
\hline & & & & $(-0.20-0.09)$ & & $(-3.87-14.74)$ & & $(-0.03-0.48)$ \\
\hline SE & & & & -.32 & & -12.03 & & -.38 \\
\hline & & & & $(-0.74-0.17)$ & & $(-31.23-6.37)$ & & $(-1.11-0.23)$ \\
\hline Success & & & & .26 & & 10.02 & & .37 \\
\hline & & & & $(0.09-0.44)$ & & $(0.55-19.78)$ & & $(-0.03-0.91)$ \\
\hline Step 2 & NS & & NS & & .02 & & .06 & \\
\hline PSP $\times$ SE & & & & & & -3.07 & & $-.33^{t}$ \\
\hline & & & & & & $(-16.06-9.86)$ & & $(-0.69-0.14)$ \\
\hline PSP $x$ & & & & & & 1.13 & & -.13 \\
\hline Success & & & & & & $(-3.68-5.34)$ & & $(-0.16-0.36)$ \\
\hline SE $x$ & & & & & & 3.14 & & .11 \\
\hline Success & & & & & & $(-6.12-13.33)$ & & $(-0.27-0.51)$ \\
\hline Step 3 & NS & & NS & & $.05^{\mathrm{t}}$ & & NS & \\
\hline PSP $x$ SE $x$ & & & & & & $3.49^{t}$ & & \\
\hline Success & & & & & & $(-0.29-6.41)$ & & \\
\hline
\end{tabular}

Note. All predictor variables are centered on the variable mean. $\Delta R^{2}$ and $\beta$ based on 1000 bootstrap samples to control for violations in normality of errors and homoscedasticity. Bias corrected and accelerated (BCa) $95 \%$ confidence intervals (CI) provided in parentheses. Week 2 represents the change between goals set on Day 8 and goals set on Day 15. WLG = Weight Loss Goal; $\mathrm{CCG}=$ Calorie Consumption Goal; EG = Exercise Goal; HHG = Healthy Habit Goal; PSP = Personal standards perfectionism; $\mathrm{SE}=$ Goal-specific Self-efficacy; Success = Goal-specific perceived success; NS = Nonsignificant.

t. $05<\mathrm{p}>.10, * \mathrm{p}<.05 . * * \mathrm{p}<.01 ., * * * \mathrm{p}<.001$ 
Table 12

Summary of Nonsignificant Simple Slopes for Two-Way Interactions on Weight-Related Goals

\begin{tabular}{|c|c|c|c|c|c|c|}
\hline \multirow{3}{*}{ Predictor } & \multicolumn{6}{|c|}{$\mathrm{HHG}$} \\
\hline & & Week & & & Veek 2 & \\
\hline & $b$ & $t$ & $p$ & $b$ & $t$ & $p$ \\
\hline $\begin{array}{l}\text { ECP x Success } \\
1 \text { SD above Mean }\end{array}$ & -0.33 & -1.65 & .105 & & & \\
\hline $\begin{array}{l}\mathrm{CPQ} \times \mathrm{SE} \\
1 \mathrm{SD} \text { below Mean }\end{array}$ & -0.16 & -1.31 & .194 & 0.10 & 1.53 & .131 \\
\hline $\begin{array}{l}\text { ECP x SE } \\
1 \text { SD below Mean }\end{array}$ & & & & 0.23 & 1.75 & .086 \\
\hline $\begin{array}{l}\text { PSP x SE } \\
1 \text { SD above Mean }\end{array}$ & & & & -0.09 & -0.47 & 639 \\
\hline
\end{tabular}

Note. Significant slope values are provided in simple slope figures and in text of results. Week 1 represents the change between goals set on Day 1 and goals set on Day 8. Week 2 represents the change between goals set on Day 8 and Day 15 . HHG = Healthy Habit Goals; SE = Goal-specific self-efficacy; Success = Goal-specific perceived success; $\mathrm{CPQ}=$ Clinical Perfectionism Questionnaire; ECP = Evaluative concerns perfectionism; PSP = Personal standards perfectionism. Final interaction of success $\mathrm{x}$ self-efficacy on HHG represents interaction entered into model with CPQ as measure of perfectionism (See Table 8). 
Table 13

Linear Regression Analysis Predicting Total Weight Loss from Perfectionism

\begin{tabular}{|c|c|c|c|c|c|c|}
\hline \multirow[b]{3}{*}{ Predictor } & \multicolumn{6}{|c|}{ Total Weight Loss } \\
\hline & \multicolumn{2}{|c|}{ CPQ } & \multicolumn{2}{|c|}{ ECP } & \multicolumn{2}{|c|}{ PSP } \\
\hline & $R^{2}$ & $\beta$ & $R^{2}$ & $\beta$ & $R^{2}$ & $\beta$ \\
\hline Perfectionism & $0.07 *$ & $-.17 *$ & NS & & NS & \\
\hline
\end{tabular}


Table 14

Description of Reasons for Raising the Bar

\begin{tabular}{|c|c|c|}
\hline Reason & Description & Select Examples \\
\hline $\begin{array}{l}\text { Attitude Toward } \\
\text { the Goal }\end{array}$ & $\begin{array}{l}\text { Any statement that refers to feelings about } \\
\text { oneself, the goal, or one's ability and } \\
\text { motivation to complete the goal (e.g., } \\
\text { stress level, motivation, self-efficacy, and } \\
\text { satisfaction). }\end{array}$ & $\begin{array}{l}\text { 1. How I feel overall, i.e., if I'm feeling ambitious, I'll set a higher } \\
\text { goal. } \\
\text { 2. Personal motivation to succeed } \\
\text { 3. Belief that I can reach them [the goals]. } \\
\text { 4. My level of excitement. } \\
\text { 5. Whether I think I can reasonably meet a future goal. }\end{array}$ \\
\hline Resources & $\begin{array}{l}\text { Any statement that refers to the resources } \\
\text { necessary to complete the goal (e.g., time, } \\
\text { work schedule, energy). }\end{array}$ & $\begin{array}{l}\text { 1. Time restraints. } \\
\text { 2. Fatigue. } \\
\text { 3. Depending on how busy I am the following week. } \\
\text { 4. If things are really settled as opposed to chaotic, I can focus more on } \\
\text { a goal. } \\
\text { 5. If work allows me to (being stuck at work might not motivate me to } \\
\text { do things to achieve my goal). }\end{array}$ \\
\hline Difficulty Level & $\begin{array}{l}\text { Any statement that refers to the difficulty } \\
\text { level of the goal (e.g., realistic, easy, } \\
\text { difficult). }\end{array}$ & $\begin{array}{l}\text { 1. How easy/difficult it was for me to achieve the goals I set. } \\
\text { 2. It depends on how easy I found it to meet my first goal. } \\
\text { 3. If I feel it is realistic. }\end{array}$ \\
\hline Success & $\begin{array}{l}\text { Any statement that refers to success, } \\
\text { achievement, or outcome of a current or } \\
\text { past goal, either with regards to the self or } \\
\text { others. }\end{array}$ & $\begin{array}{l}\text { 1. How many others achieved the same goal. } \\
\text { 2. If I exceeded my goal. } \\
\text { 3. The outcome of the goal. }\end{array}$ \\
\hline Self-Improvement & $\begin{array}{l}\text { Any statement that refers to setting more } \\
\text { challenging goals, aiming for self- } \\
\text { improvement, or reasons why the previous } \\
\text { goal was not sufficient. }\end{array}$ & $\begin{array}{l}\text { 1. Just ready to take on the next challenge. } \\
\text { 2. Improving myself } \\
\text { 3. There is always room for improvement! You can never be too } \\
\text { healthy. } \\
\text { 4. Results were not enough. } \\
\text { 5. The need to obtain better results. } \\
6 \text {. Want to see if I can go further and test myself. }\end{array}$ \\
\hline
\end{tabular}


Table 15

Interrater Agreement for Qualitative Analysis Total Statements \# Agreement $\%$ Agreement

Attitude 24 18 19.5

81.2

Resources

13

18

81.2

Difficulty

11

Success

6

9

69.2

Self-Improvement

7.5

68.2

5

83.3

\begin{tabular}{l} 
Total $72 \quad 59$ \\
\hline Note. Of 49 participants, 2 participants did not provide a response. Responses from individual participants could \\
include multiple statements. Total statements = the number of statements initially coded within each respective \\
category. \# Agreement = the number of statements which were coded the same as the initial coder (Total \\
Statements). Full points were attributed to statements that were completely congruent with initial coder. Half points \\
were attributed to statements that were $50 \%$ congruent with the initial coder (e.g., first part of statement coded the \\
same as the initial coder, second part of statement coded differently). \% Agreement = the percentage of statements \\
which were coded the same as the initial coder (Total Statements). One statement: "Be Healthy" was coded as not \\
fitting into a category by the initial coder, and was coded as Self-Improvement by Coder 2, but was not included in \\
the above table.
\end{tabular}


Table 16

Frequency of GGSQ StanD and CPQ Scores in Top Third of GGSQ StanI Participants

\begin{tabular}{|c|c|c|c|c|c|}
\hline \multicolumn{3}{|c|}{ GGSQ StanD } & \multicolumn{3}{|c|}{$\mathrm{CPQ}$} \\
\hline Valid Score & Frequency & $\begin{array}{c}\text { Cumulative } \\
\%\end{array}$ & $\begin{array}{l}\text { Valid } \\
\text { Score }\end{array}$ & Frequency & $\begin{array}{c}\text { Cumulative } \\
\%\end{array}$ \\
\hline 6 & 1 & 2.0 & 17 & 2 & 4.1 \\
\hline 8 & 1 & 4.1 & 20 & 1 & 6.1 \\
\hline 9 & 1 & 6.1 & 21 & 1 & 8.2 \\
\hline 10 & 2 & 10.2 & 23 & 1 & 10.2 \\
\hline 12 & 3 & 16.3 & 24 & 4 & 18.4 \\
\hline 13 & 2 & 20.4 & 25 & 4 & 26.5 \\
\hline 14 & 1 & 22.4 & 27 & 2 & 30.6 \\
\hline 16 & 1 & 24.5 & 28 & 10 & 51.0 \\
\hline 17 & 3 & 30.6 & 29 & 4 & 59.2 \\
\hline 18 & 4 & 38.8 & 30 & 2 & 63.3 \\
\hline 19 & 4 & 46.9 & 31 & 4 & 71.4 \\
\hline 20 & 2 & 51.0 & 32 & 4 & 79.6 \\
\hline 21 & 3 & 57.1 & 33 & 3 & 85.7 \\
\hline 22 & 3 & 63.3 & 34 & 2 & 89.8 \\
\hline 23 & 2 & 67.3 & 35 & 2 & 93.9 \\
\hline 24 & 4 & 75.5 & 38 & 1 & 95.9 \\
\hline 25 & 3 & 81.6 & 39 & 1 & 98.0 \\
\hline 28 & 2 & 85.7 & 40 & 1 & 100.0 \\
\hline 20 & 1 & 87.8 & & & \\
\hline 30 & 2 & 91.8 & & & \\
\hline 31 & 2 & 95.9 & & & \\
\hline 33 & 1 & 98.0 & & & \\
\hline 41 & 1 & 100.0 & & & \\
\hline
\end{tabular}

Note. GGSQ = General Goal-setting Questionnaire; $\mathrm{StanD}=$ Standard Dissatisfaction subscale; $\mathrm{CPQ}=$ Clinical Perfectionism Questionnaire; Valid Score $=$ Scores where at least one participant received that total score. GGSQ StanD ranged from 6-41 out of a total of 42 . CPQ ranged from 17 to 40 out of a total 48. 
Table 17

Responses of Participants that Scored in the Top Third of the GGSQ StanI and GGSQ StanD or the CPQ

\begin{tabular}{|c|c|c|}
\hline & GGSQ StanD Statements & CPQ Statements \\
\hline \multicolumn{3}{|l|}{ Category } \\
\hline Attitude & $\begin{array}{l}\text { 1. My mood/How I feel about myself } \\
\text { 3. If I wasn't very stressed out while meeting the original } \\
\text { goal } \\
\text { 4. Satisfaction } \\
\text { 5. If I want to work harder }\end{array}$ & $\begin{array}{l}\text { 1. My mood/Based on feelings/Whether I feel good/How I feel } \\
\text { about myself } \\
\text { 2. Stress while trying to accomplish the goal. } \\
\text { 3. Satisfaction } \\
\text { 4. Belief that I can reach them [the goals]. } \\
\text { 5. If I want to work harder }\end{array}$ \\
\hline Resources & $\begin{array}{l}\text { 1. How busy my schedule is } \\
2 \text {. If I have the time }\end{array}$ & $\begin{array}{l}\text { 1. How busy my schedule is/Schedule } \\
\text { 2. Resources } \\
\text { 3. If I have the time/Time restraints/Time } \\
\text { 4. Fatigue } \\
\text { 5. Soreness } \\
\text { 6. Perspiration }\end{array}$ \\
\hline Difficulty & $\begin{array}{l}\text { 1. When it was too easy for me to stick within the daily } \\
\text { recommended caloric intake for weight loss }\end{array}$ & $\begin{array}{l}\text { 1. How difficult it was to achieve. } \\
\text { 2. If the goal was difficult. } \\
\text { 3. If it's realistic. }\end{array}$ \\
\hline Success & $\begin{array}{l}\text { 1. If I exceeded my goal. } \\
\text { 2. Meeting the previous goal. }\end{array}$ & $\begin{array}{l}\text { 1. If I exceeded my goal. } \\
\text { 2. Meeting my previous goal. } \\
\text { 3. Considering whether I just barely achieved my goal or surpassed } \\
\text { my goal without too much difficulty. } \\
\text { 4. Impact of previous goal. } \\
\text { 5. How many others achieved the same goal. }\end{array}$ \\
\hline $\begin{array}{l}\text { Self- } \\
\text { Improvement }\end{array}$ & 1. The need to obtain better results. & $\begin{array}{l}\text { 1. There is always room for improvement! You can never be too } \\
\text { healthy. }\end{array}$ \\
\hline
\end{tabular}


Table 18

Remaining Statements for Self Improvement with Accompanying GGSQ Factor and CPQ Scores

\begin{tabular}{|c|c|c|c|}
\hline GGSQ StanI & GGSQ StanD & CPQ & Statement \\
\hline 21 & 12 & 23 & Just ready to take on the next challenge. \\
\hline 19 & 20 & 17 & Improving myself. \\
\hline 17 & 23 & 29 & Results were not enough. \\
\hline 15 & 17 & 25 & Want to see if I can go further and test myself \\
\hline
\end{tabular}

Note. GGSQ = General Goal-setting Questionnaire StanI = Standard Increase subscale; $\mathrm{StanD}=$ Standard Dissatisfaction subscale; $\mathrm{CPQ}=$ Clinical

Perfectionism Questionnaire. 


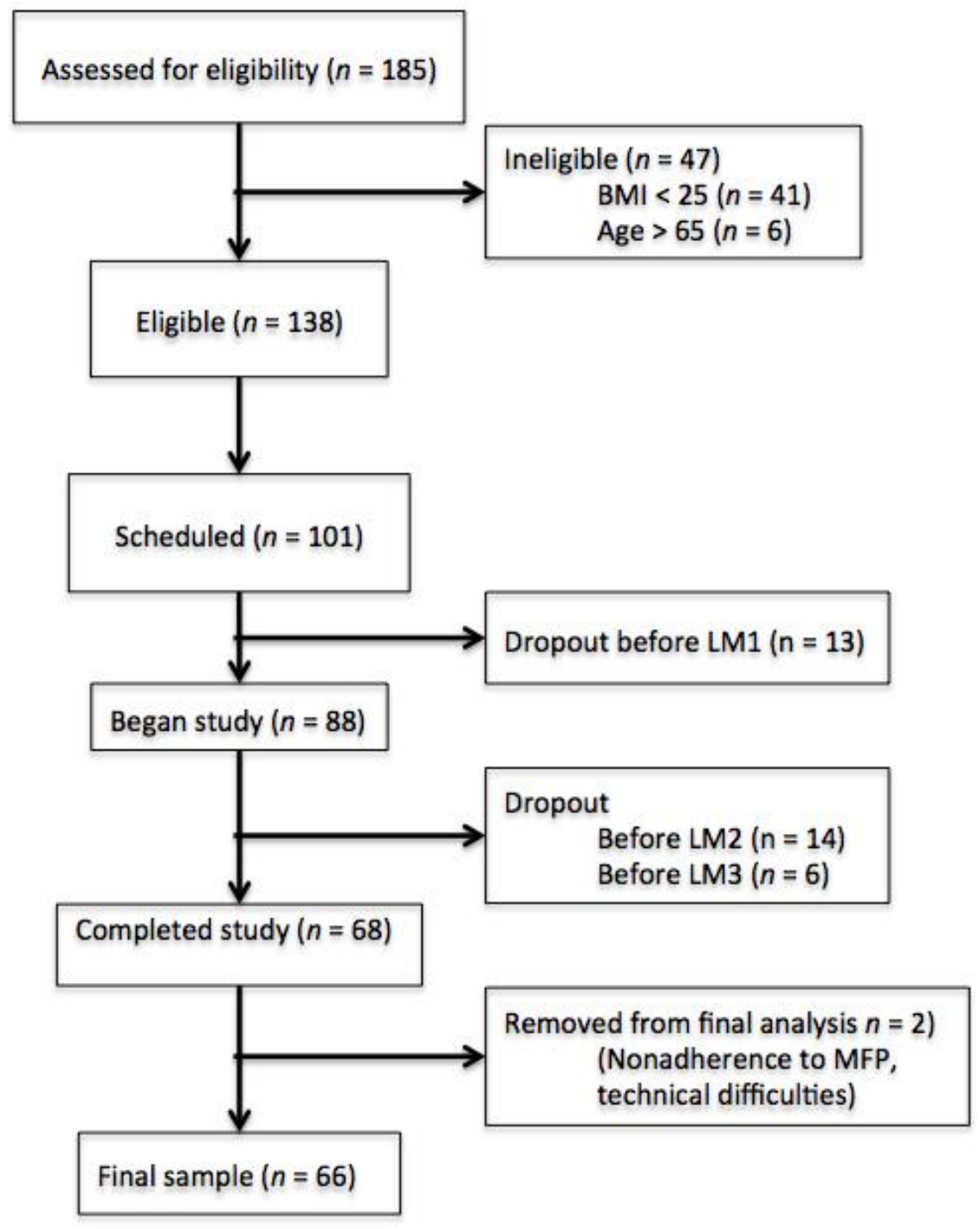

Figure 1. Response rate throughout recruitment and testing process. 
RAISING THE BAR

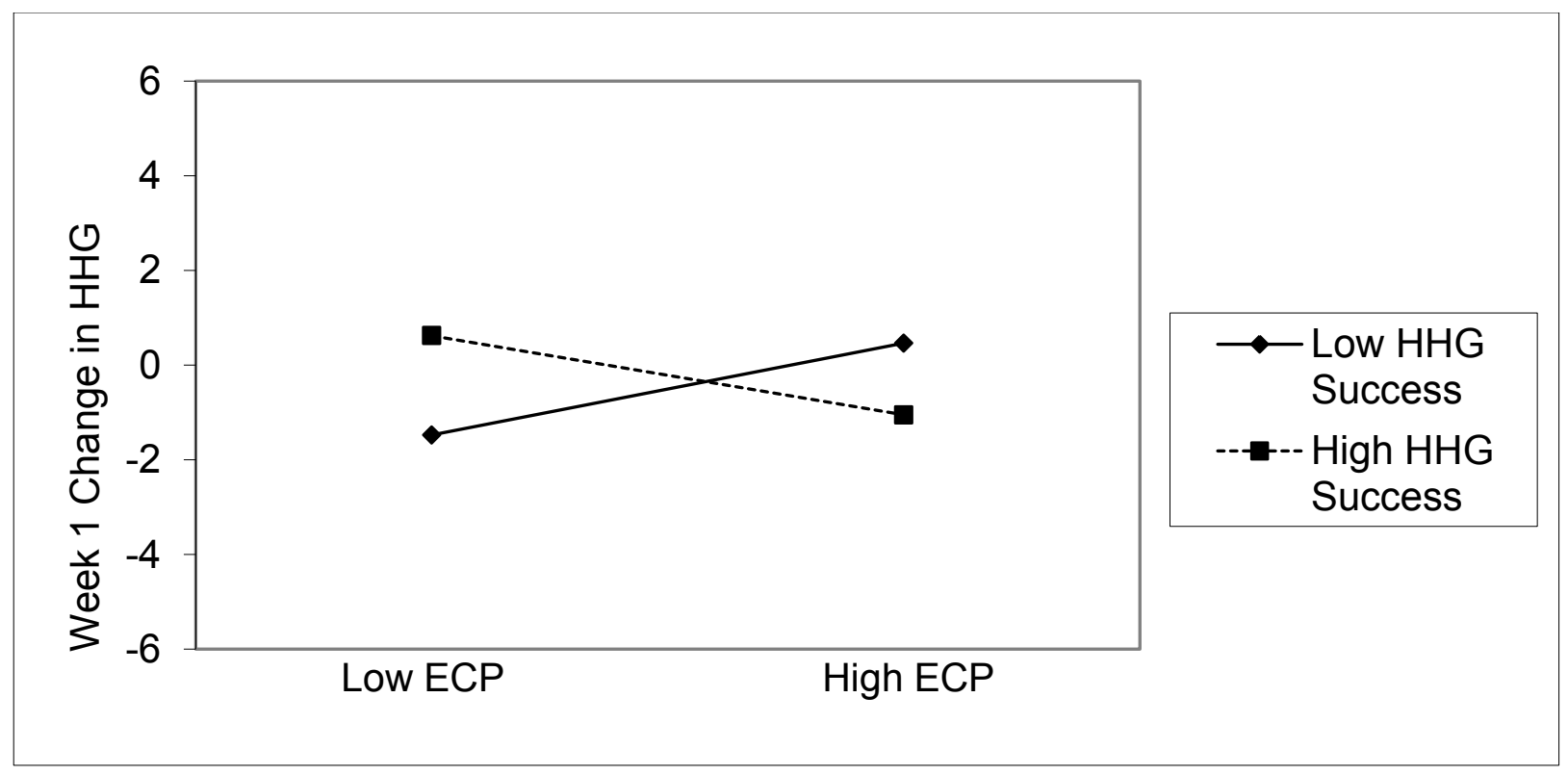

Figure 2. Simple slopes analysis of ECP by HHG success on Week 1 HHG change.

Week 1 Change in HHG ranges from -6 to 5 change in goals selected from Day 1 to Day 8 . HHG = Healthy Habit Goals, ECP = Evaluative concerns perfectionism. Slopes based on centered and bootstrapped variables from Table 7. Low HHG self-efficacy ( $1 S D$ below mean of HHG average self-efficacy): $b=0.38, t=2.18, p=.033$. High HHG self-efficacy (1 SD above the mean of HHG average self-efficacy: $b=-.33, t=-1.65$, NS. 
RAISING THE BAR

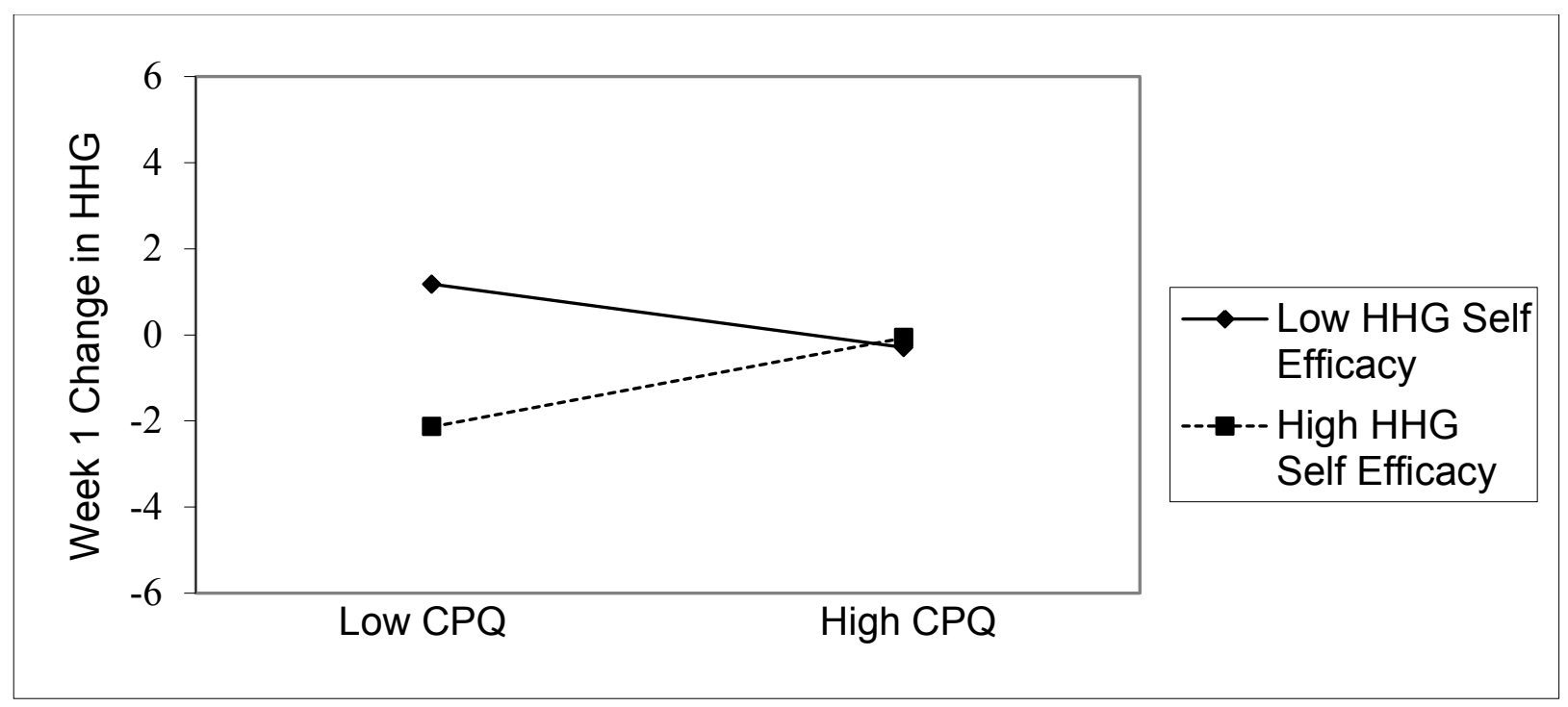

Figure 3. Simple slopes analysis of CPQ by average HHG self-efficacy on Week 1 HHG change.

Week 1 Change in HHG ranges from -6 to 5 change in goals selected from Day 1 to Day 8 . HHG $=$ Healthy Habit Goals, $\mathrm{CPQ}=$ Clinical Perfectionism Questionnaire. Slopes based on centered and bootstrapped variables from Table 6. Low HHG self-efficacy (1 $S D$ below mean of HHG average self-efficacy): $b=0.10, t=-1.53$, NS. High HHG self-efficacy (1SD above the mean of HHG average self-efficacy: $b=0.22, t=2.68, p=.010$. 
RAISING THE BAR

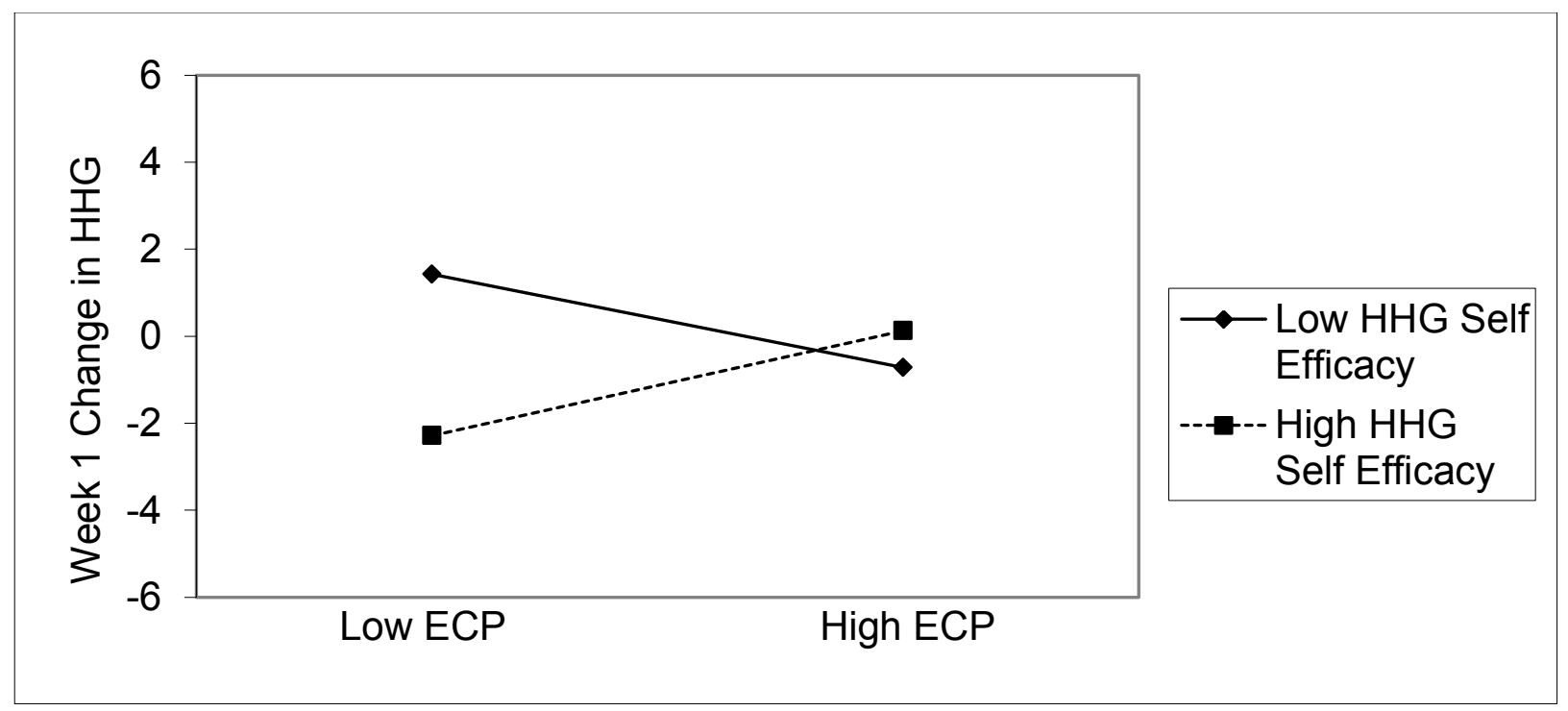

Figure 4. Simple slopes analysis of ECP by average HHG self-efficacy on Week 1 HHG change.

Week 1 Change in HHG ranges from -6 to 5 change in goals selected from Day 1 to Day 8 . HHG = Healthy Habit Goals, ECP= Evaluative concerns perfectionism. Slopes based on centered and bootstrapped variables from Table 7. Low HHG self-efficacy (1 $S D$ below mean of HHG average self-efficacy): $b=-0.42, t=-2.16, p=.035$. High HHG self-efficacy ( $1 S D$ above the mean of HHG average self-efficacy: $b=0.47, t=3.07, p=.043$. 


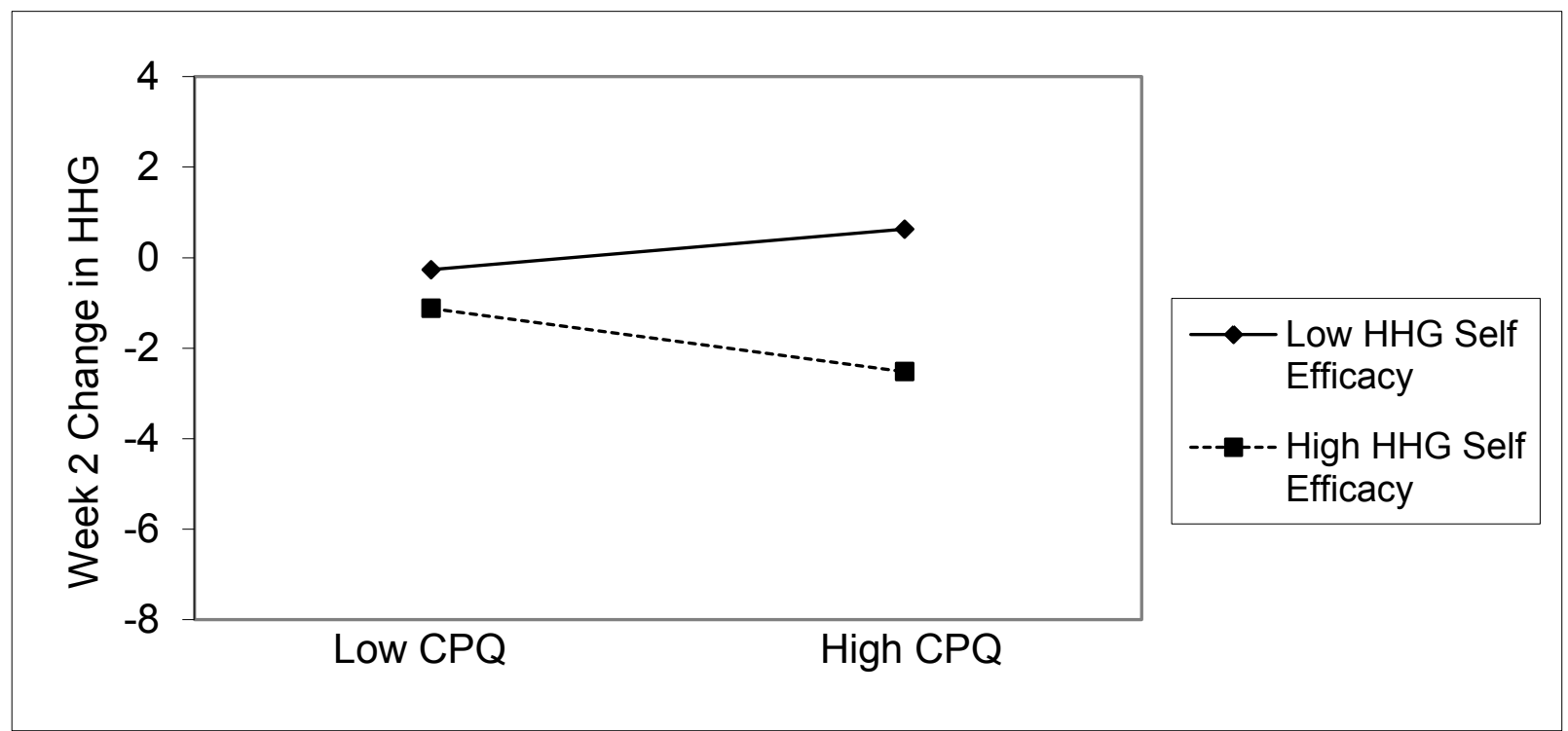

Figure 5. Simple slopes analysis of CPQ by average HHG self-efficacy on Week 2 HHG change.

Week 1 Change in HHG ranges from -8 to 3 change in goals selected from Day 8 to Day 15 . HHG $=$ Healthy Habit Goals, CPQ = Clinical Perfectionism Questionnaire. Slopes based on centered and bootstrapped variables from Table 9. Low HHG self-efficacy (1 SD below mean of HHG average self-efficacy): $b=-0.001, t=-0.03$, NS. High WLG self-efficacy ( $1 S D$ above the mean of HHG average self-efficacy: $b=-0.15, t=-2.41, p=.019$. 


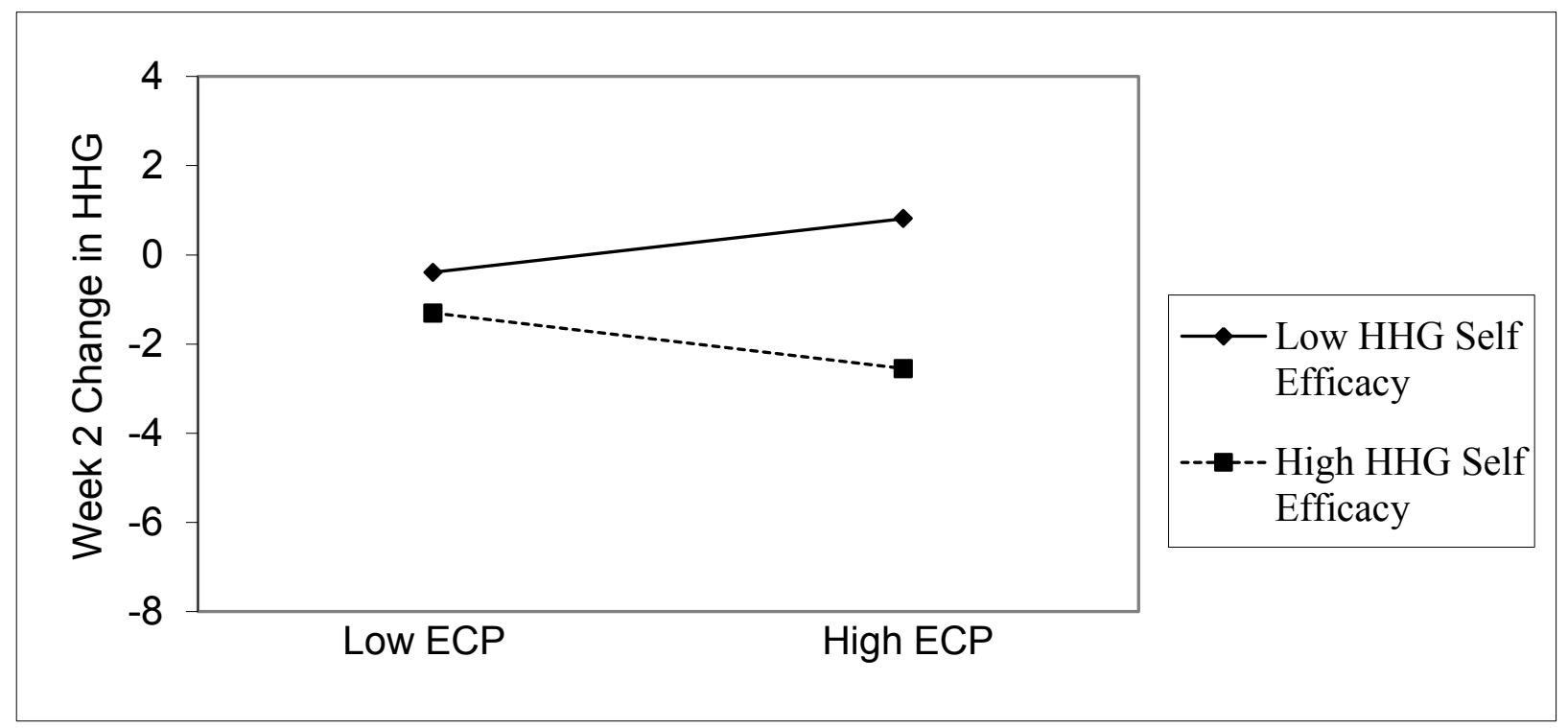

Figure 6. Simple slopes analysis of CPQ by average HHG self-efficacy on Week 2 HHG change.

Week 1 Change in HHG ranges from -8 to 3 change in goals selected from Day 8 to Day 15 . HHG $=$ Healthy Habit Goals, ECP = Evaluative concerns perfectionism. Slopes based on centered and bootstrapped variables from Table 10. Low HHG self-efficacy ( $1 S D$ below mean of HHG average self-efficacy): $b=0.23, t=1.75$, NS. High WLG self-efficacy (1SD above the mean of HHG average self-efficacy: $b=-0.24, t=-2.13, p=.038$. 


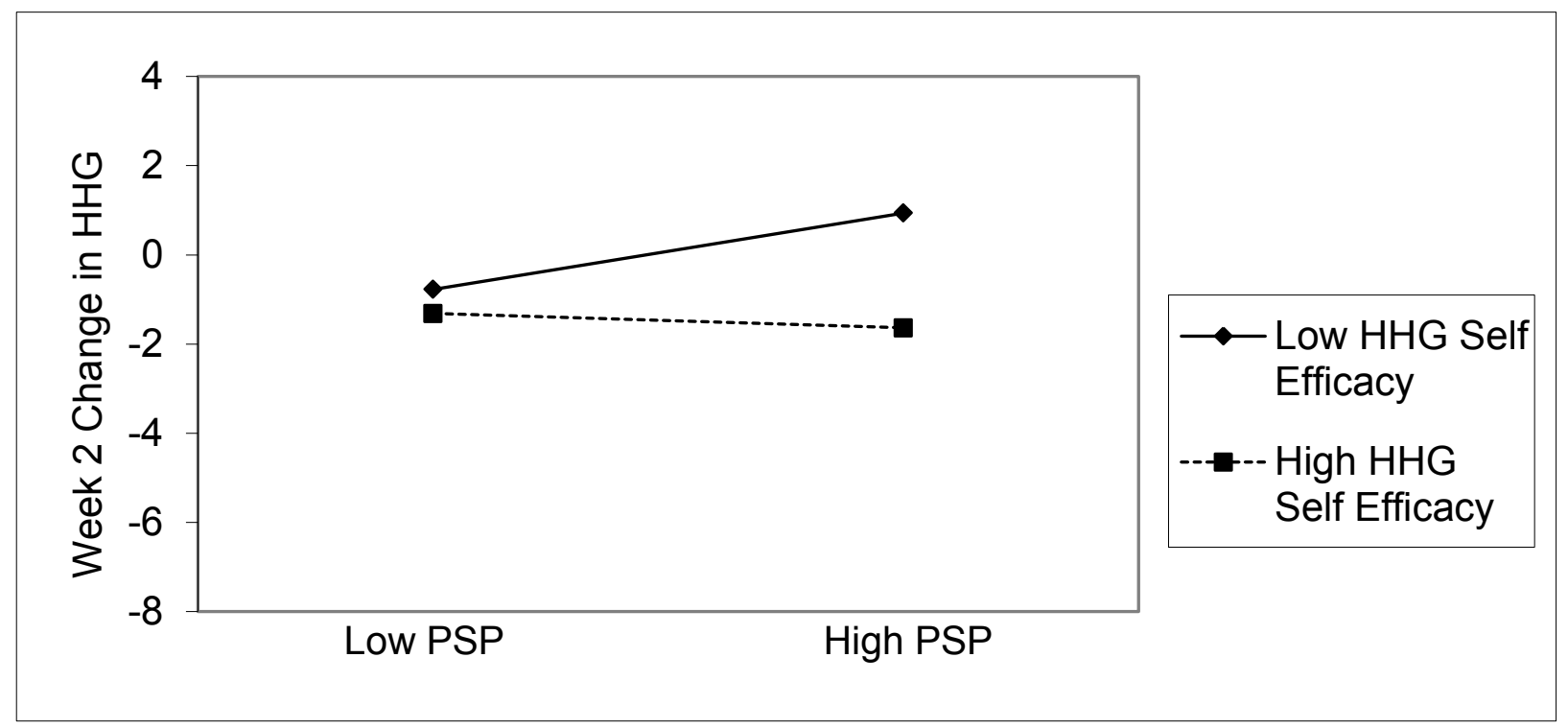

Figure 7. Simple slopes analysis of PSP by average HHG self-efficacy on Week 2 HHG change.

Week 1 Change in HHG ranges from -8 to 3 change in goals selected from Day 8 to Day 15 . HHG $=$ Healthy Habit Goals, PSP $=$ Personal standards perfectionism. Slopes based on centered and bootstrapped variables from Table 11 . Low HHG self-efficacy ( $1 S D$ below mean of HHG average self-efficacy): $b=0.50, t=2.51, p=.015$. High WLG self-efficacy ( $1 S D$ above the mean of HHG average self-efficacy: $b=-0.09, t=-0.47$, NS. 


\section{Appendix A}

\section{Goal-setting Questionnaire (GSQ) - Week 1}

Setting specific and measureable weight loss goals has been shown to be an effective weight loss strategy when combined with self-monitoring food intake. Therefore, in addition to selfmonitoring what you eat every day using the My Fitness Pal food diary app, this questionnaire provides you with an additional weight loss strategy by asking you to set specific and measurable goals once each week. These goals include weight loss, calorie consumption, exercise, and healthy habit goals. At the end of each week, you will (1) be provided with a reminder of the goals that you set, (2) evaluate whether you met your goals, and (3) decide whether you would like to change or revise your goals for the upcoming week.

Weight:

Height:

My Fitness Pal asks you to identify how many pounds you would like to lose in total while completing the self-monitoring food diary. Please indicate your total weight loss goal below:

I wish to lose lbs. in total

\section{Weight loss Goals}

\section{My Fitness Pal Weekly Weight loss Goal}

My Fitness Pal asks you to identify how many pounds you would like to lose per week while completing the self-monitoring food diary. For the upcoming week, which of the following did you select as your weight loss goal?

$0.5 \mathrm{lbs} . /$ week

1lb./week

$1.51 \mathrm{bs} . /$ week

2lbs./week

How successful do you expect to be in meeting your My Fitness Pal's weekly weight loss goal this upcoming week?
1
2

3

4

5

6

7

Not at all

Completely

successful

successful 


\section{Personal Weekly Weight loss Goal}

If My Fitness Pal allowed you to select a different weekly weight loss goal (i.e. more than 2 lbs./week), would you?

\section{YES NO}

If YES, indicate the number of lbs./week you would like to lose

How successful do you expect to be in meeting your personal weekly weight loss goal this upcoming week?

1

Not at all successful
$2 \quad 3$

4

5

6

Completely

successful

\section{Calorie Consumption Goals}

Depending on your BMI, and your weight loss goals (e.g., losing 1.5lbs./week), My Fitness Pal will calculate the number of calories/day you should consume to meet your goal.

How many calories/day did My Fitness Pal suggest you consume? calories/day

If you could select the number of calories/day to consume, how many calories would you select? calories/day

Even though My Fitness Pal has suggested the number of calories/day you should consume to meet your weight loss goal, you have the ability to exceed this number of calories depending on which foods you eat.

How many days this week would you like to be at or below the suggested number of My Fitness Pal calories:

days (Please select a number between 1 and 7)

How successful do you expect to be in meeting your calorie consumption goal this upcoming week (i.e., do you expect to eat at or below the suggested number of My Fitness Pal calories for the number of days you indicated)?

1

Not at all successful
4

5

6 7 Completely successful 


\section{Exercise Goals}

In addition to tracking what you eat, My Fitness Pal will allow you to enter your exercise goals by asking how much exercise you would like to do this week, and how many minutes each exercise session should last.

How many days this week would you like to exercise days (Please select a number between 1 and 7)

How long would you like each of your exercise sessions to be? minutes

Please list what types of activities you do when you workout (e.g., swimming, running, weights, etc.)

1.

2.

3.

4.

How successful do you expect to be in meeting your exercise goal this upcoming week?

1

Not at all successful
4

5

6 7 Completely successful 


\section{Healthy Habit Goals}

In your personal experience, you may have certain bad habits that you believe contribute to your weight gain. Perhaps you eat a snack late at night before bed, even when you're still full from dinner. Or maybe you choose sugary drinks over water. Below is a list of healthy habit goals.

Please select at least one goal from the list below that you would like to follow this week by adding a checkmark beside the appropriate goal. If you would like, you can select multiple goals. Keep track of your progress, because you will be asked how successful you were at keeping your healthy habit goals each week.

\section{Healthy Habit Goals}

1. Eat lunch and dinner using the plate method $(1 / 2$ plate $=$ vegetables, $1 / 4$ plate $=$ meat or protein, $1 / 4$ plate grains or starchy food)

Refer to The Plate Method handout for more information and for examples of protein and starch

2. $\quad$ Eat meals with a minimum of 3 food groups (Dairy, grain, protein, fruits and vegetables)

3. Avoid eating at restaurants (e.g., pack your lunch, eat meals prepared at home)

4. Eat food off of personal plates, bowls, and dishware instead of serving platters, bags, and containers.

5. Avoid distractions while eating (e.g., working, watching TV, surfing the web, reading a book, playing with your phone)

6. Go grocery shopping with a list and a full stomach (**Only select this goal if you will go grocery shopping at least once this week)

7. Drink water instead of sugary drinks (e.g., juice, beer, pop)

8. $\quad$ Avoid fried foods (e.g., French fries, chicken fingers)

9. Move to a food free location once you've finished your meal *

10. Avoid second helpings by removing serving dishes from the table

11. Eat your meals sitting at a table *

12. Eat one dark green and one orange vegetable per day *

13. Eat a minimum number of fruits and vegetable servings per day* Indicate serving number__ (recommended 5 servings)

14. Avoid eating treats or snacks after dinner or before bed.

15. Slow down the pace of eating meals (e.g. put down your fork between bites)

16. Other:

17. Other:

18. Other:

19. Other:

20. Other:

* From the Cognitive Behavioral Workbook for Weight Management (Laliberté, McCabe, \& Taylor, 2009) 
How successful do you expect to be in following the healthy habit goal(s) you selected for the upcoming week?

12

Not at all successful
3

4

5

6 7 Completely successful

\section{Overall Accuracy of Food Diary}

Some people find it difficult to accurately record their food intake every day.

Using the following scale, please indicate how accurately you completed your food diary this week:

1

Not at all

accurate
3

4

5

6 Completely accurate 


\section{Goal-Setting Questionnaire (GSQ) - Week 2/3}

Setting specific and measureable weight loss goals has been shown to be an effective weight loss strategy when combined with self-monitoring food intake. Therefore, in addition to selfmonitoring what you eat every day using the My Fitness Pal food diary app, this questionnaire provides you with an additional weight loss strategy by asking you to set specific and measurable goals once each week. These goals include weight loss, calorie consumption, exercise, and healthy habit goals. At the end of each week, you will (1) be provided with a reminder of the goals that you set, (2) evaluate whether you met your goals, and (3) decide whether you would like to change or revise your goals for the upcoming week.

My Fitness Pal asks you to identify how many pounds you would like to lose in total while completing the self-monitoring food diary. Please indicate your total weight loss goal below:

\section{Weight Loss Goals}

\section{My Fitness Pal Weekly Weight loss Goal}

What was your weight last week (see Summary of Goals)

What is your weight this week?

My Fitness Pal asks you to identify how many pounds you would like to lose per week while completing the self-monitoring food diary. Remembering the number of pounds/week you wished to lose last week according to My Fitness Pal's options (see Summary of Goals), how successful were you in meeting My Fitness Pal's weekly weight loss goal (i.e., did you lose the intended amount of weekly weight)?

1

Not at all successful
3

4

5

6 7 Completely successful

For the upcoming week, which of the following do you want to select as your weight loss goal?

$0.5 \mathrm{lbs} . /$ week

11b./week

1.5lbs./week

2lbs./week

How successful do you expect to be in meeting your My Fitness Pal's weekly weight loss goal this upcoming week? 
RAISING THE BAR

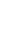

2

3

4

5

6

7

Not at all

successful

Completely

successful

\section{Personal Weekly Weight loss Goal}

Last week you were asked if you would set a different weekly weight loss goal if allowed by $M y$ Fitness Pal (i.e., more than $2 \mathrm{lbs}$./week). If you answered YES to that question, remembering the number of pounds/week you wished to lose last week independent of My Fitness Pal's options (See Summary of Goals), how successful were you in meeting this alternate weekly weight loss goal (i.e., did you lose the intended amount of weekly weight)?
1
2
3
4
5
6
7

Not at all Completely successful successful

For the upcoming week, if My Fitness Pal allowed you to set another weekly weight loss goal, would you?

YES NO

If YES, indicate the number of lbs./week you would like to lose

How successful do you expect to be in meeting your personal weekly weight loss goal this upcoming week?

1

Not at all successful
4

5

6

7

Completely

successful 


\section{Calorie Consumption Goals}

Depending on your BMI, and your weight loss goals (e.g., losing 1.5lbs./week), My Fitness Pal will calculate the number of calories/day you should consume to meet your goal.

How many calories/day did My Fitness Pal suggest you consume?

calories/day

Even though My Fitness Pal has suggested the number of calories/day you should consume to meet your weight loss goal, you have the ability to exceed this number of calories depending on which foods you eat.

Remembering the number of days during the past week you wanted to be at or below the suggested number of calories (See Summary of Goals), how successful were you in meeting your goal?

1

Not at all successful
45

56 7 Completely successful

For the upcoming week, if you could select the number of calories/day to consume, how many calories would you select? calories/day

For the upcoming week, how many days this week would you like to be at or below the suggested number of calories:

days (Please select a number between 1 and 7)

How successful do you expect to be in meeting your calorie consumption goal this upcoming week (i.e., do you expect to eat at or below the suggested number of My Fitness Pal calories for the number of days you indicated)?

1

Not at all successful
3
5

6

Completely

successful 


\section{Exercise Goals}

In addition to tracking what you eat, My Fitness Pal will allow you to enter your exercise goals by asking how much exercise you would like to do this week, and how many minutes each exercise session should last.

Remembering the total amount of time (days x minutes) during the past week you wanted to exercise (See Summary of Goals), how successful were you at meeting your goal?
1
2
3
4
5
6
7

Not at all

successful Completely successful

For the upcoming week, how many days would you like to exercise?

days (Please select a number between 1 and 7)

For the upcoming week, how long would you like each of your exercise sessions to be? minutes

Please list what types of activities you do when you workout (e.g., swimming, running, weights, etc.)

1.

2.

3.

4.

How successful do you expect to be in meeting your exercise goal this upcoming week?

1

Not at all

successful
4

5

6

7

Completely successful 


\section{Healthy Habit Goals}

In your personal experience, you may have certain bad habits that you believe contribute to your weight gain. Perhaps you eat a snack late at night before bed, even when you're still full from dinner. Or maybe you choose sugary drinks over water.

Remembering the healthy habit goals you wanted to follow during the past week (See Summary of Goals), how successful were you in following the goals you selected?
1
2
3
4
5
6
7

Not at all

Completely

successful

successful

For the upcoming week, please select at least one goal from the list below that you would like to follow by adding a checkmark beside the appropriate goal. It can be the same goal or a different goal from the previous week. If you would like, you can select multiple goals. Keep track of your progress, because you will be asked how successfully you were at keeping your healthy habit goals each week.

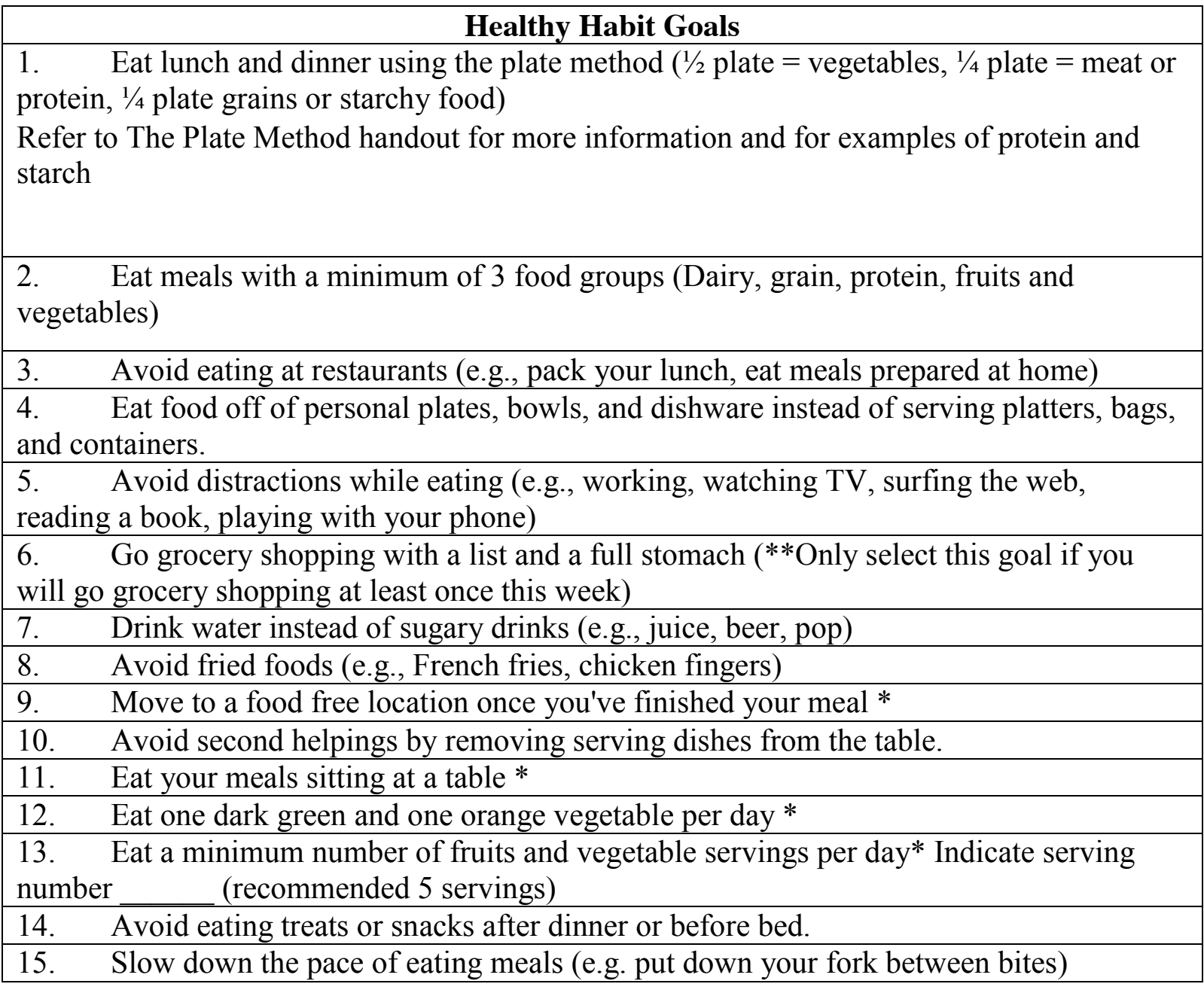




\begin{tabular}{|ll|}
\hline 16. & Other: \\
\hline 17. & Other: \\
\hline 18. & Other: \\
\hline 19. & Other: \\
\hline 20. & Other: \\
\hline
\end{tabular}

* From the Cognitive Behavioral Workbook for Weight Management (Laliberté, McCabe, \& Taylor, 2009)

How successful do you expect to be in following the healthy habit goal(s) you selected for the upcoming week?

12

Not at all successful
3

4

5

6 7 Completely successful

\section{Overall Accuracy of Food Diary}

Some people find it difficult to accurately record their food intake every day.

Using the following scale, please indicate how accurately you completed your food diary this week:

1

Not at all accurate
2

3
4

5

6

Completely accurate 


\section{Appendix B}

\section{Part 1}

\section{General Goal-setting Questionnaire}

A goal is a specific objective or aim that someone wants to achieve or accomplish. Goals can be set in a number of different life domains including school (e.g., finishing a project or passing an exam), work (e.g., getting a promotion or landing a client), eating or appearance (e.g., losing weight or exercising more frequently), or sports, hobbies or activities (e.g., finishing a painting or winning a tennis match). The following are a list of statements that describe your attitudes and feelings towards meeting your goals. These statements refer to any life domain or category in which you find yourself setting goals. Indicate the extent to which you agree or disagree with the following statements.

12

Strongly

Disagree
3

4

Neutral
5

6 7 Strongly Agree

1. When I meet a goal, I set more challenging or difficult goals the next time.

2. I feel the need to set more challenging goals after meeting a previous goal.

3. I move onto more challenging or difficult goals after meeting a goal.

4. Meeting my goal means that it must have been too easy.

5. When I meet a goal, I think that I should have aimed for a bigger goal.

6. Meeting my goal means that it wasn't sufficiently challenging or difficult to begin with.

7. Meeting my goal means that I should have set a more challenging or difficult goal to begin with.

8. Even if I initially feel good about accomplishing a goal, I soon feel that my goal was not sufficiently challenging.

9. My sense of accomplishment never lasts long after meeting a goal.

\section{Part 2}

What factors determine whether you decide to set a more challenging goal for yourself after successfully meeting a previous goal? 


\section{Appendix C}

\section{Summary of Goals - Week 1/2/3}

Below is a summary of the weight loss, calorie consumption, exercise, and healthy habit goals you set the previous week. Please use this summary to remind yourself of your goals for this week and to complete the Goal-setting Questionnaire for the upcoming week. Specifically, use the information below to (1) evaluate whether you met your goals for the past week, and (2) decide whether you would like to change or revise your goals for the upcoming week.

BMI:

Current weight:

\section{My Fitness Pal Weekly Weight loss Goal}

\section{Weight loss Goals}

Last week you weighed:

My Fitness Pal asks you to identify how many pounds you would like to lose per week while completing the selfmonitoring food diary.

According to My Fitness Pal's options, you selected lbs./week

\section{Personal Weekly Weight loss Goal}

When asked if you would select a different weekly weight loss goal if My Fitness Pal allowed (i.e. more than 2 $\mathrm{lbs} /$ week), you answered: (YES/NO)

Specifically, you indicated that you would like to lose lbs./week independent of My Fitness Pal's options.

\section{Calorie Consumption Goals}

My Fitness Pal suggested you consume calories/day

When asked how many calories/day you would consume if you could choose, you selected calories/day

When asked how many days during the week you would like to be at or below the suggested number of calories, you selected days

You wished to exercise

\section{Exercise Goals}

days for minutes each time

\section{Healthy Habit Goals}

You selected the following healthy habit goals to follow:

1.

2.

3. 
RAISING THE BAR

\section{References}

Aardoom, J. J., Dingemans, A. E., Slof Op't Landt, M. C. T., \& Van Furth, E. F. (2012). Norms and discriminative validity of the Eating Disorder Examination Questionnaire (EDEQ). Eating Behaviors, 13, 305-309.

Aiken, L. S., \& West, S. G. (1991). Multiple regression: Testing and interpreting interactions. Thousand Oaks, CA: Sage.

Alden, L. E., Bieling, P. J., \& Wallace, S. T. (1994). Perfectionism in an interpersonal context: A self-regulation analysis of dysphoria and social anxiety. Cognitive Therapy and Research, 18, 297-316.

American Gastroenterological Association (2002). American Gastroenterological Association medical position statement on obesity. Gastroenterology, 123, 879-881.

American Psychiatric Association (2013). Diagnostic and statistical manual of mental disorders (5th ed.). Arlington, VA: American Psychiatric Press.

Anderson, D. A., Lundgren, J. D., Shapiro, J. R., \& Paulosky, C. A. (2004). Assessment of eating disorders: Review and recommendations for clinical use. Behavior Modification, 28, 763782.

Antony, M. M., Bieling, P. J., Cox, B. J., Enns, M. W. \& Swinson, R. P. (1998). Psychometric properties of the 42-item and 21-item versions of the Depression Anxiety Stress Scales in clinical groups and a community sample. Psychological Assessment, 10, 176-181.

Antony, M. M., Purdon, C. L., Huta, V., \& Swinson, R. P. (1998). Dimensions of perfectionism across the anxiety disorders. Behaviour Research and Therapy, 36, 1143-1154.

Atlantis, E., \& Ball, K. (2008). Association between weight perception and psychological distress. International Journal of Obesity, 32, 315-723. 
Bagozzi, R. P., \& Edwards, E. A. (2000). Goal-striving and the implementation of goal intentions in the regulation of body weight. Psychology and Health, 15, 255-270.

Baker, R. C., \& Kirschenbaum, D. S. (1993). Self-monitoring may be necessary for successful weight control. Behavior Therapy, 24, 377-394.

Baker, R. C., \& Kirschenbaum, D. S. (1998). Weight control during the holidays: Highly consistent self-monitoring as a potentially useful coping mechanism. Health Psychology, $17,367-370$.

Bandura, A. (2006). Guide for constructing self-efficacy scales. In F. Pajares, \& T. Urdan (Eds.), Self-efficacy beliefs of adolescents (pp. 307-337). Charlotte, NC: Information Age Publishing.

Bandura, A. (1998). Health promotion from the perspective of social cognitive theory. Psychology and Health, 13, 623-649.

Bandura, A. (1977). Self-efficacy: Toward a unifying theory of behavioral change. Psychological Review, 84, 191-215.

Bandura, A. (1986). Social foundations of thought and action: A social cognitive theory. Englewood Cliffs, NJ: Prentice Hall.

Bardone-Cone, A. (2007). Self-oriented and socially prescribed perfectionism dimensions and their associations with disordered eating. Behaviour Research and Therapy, 45, 19771986.

Bardone-Cone, A. M., \& Boyd, C. A. (2007). Psychometric properties of eating disorder instruments in black and white young women: Internal consistency, temporal stability, and validity. Psychological Assessment, 19, 356-362. 
Bardone-Cone, A., Brownstone, L. M., Higgins, M. K., Harney, M. B., \& Fitzsimmons-Craft, E. (2012). Predicting difficulties controlling overeating and drinking when experiencing negative affect in undergraduate women. Journal of Social and Clinical Psychology, 31, $1051-1073$.

Bardone-Cone, A., Sturm, K., Lawson, M., Robinson, D., \& Smith, R. (2010). Perfectionism across stages of recovery from eating disorders. International Journal of Eating Disorders, $43,139-148$.

Bastiani, A. M., Rao, R., Weltzin, T., \& Kaye, W. H. (1995). Perfectionism in anorexia nervosa. International Journal of Eating Disorders, 17, 147-152.

Besser, A., Flett, G.L., \& Hewitt, P.L. (2004). Perfectionism, cognition, and affect in response to performance failure vs success. Journal of Rational-Emotive and Cognitive-Behavior Therapy, 22, 301-328.

Berg, K. C., Peterson, C. B., Frazier, P., \& Crow, S. J. (2012) Psychometric evaluation of the eating disorder examination and eating disorder examination-questionnaire: A systematic review of the literature. International Journal of Eating Disorders, 45, 428-438.

Bieling, P. J., \& Alden, L. E. (1997). The consequences of perfectionism for patients with social phobia. British Journal of Clinical Psychology, 36, 387-395.

Bieling, P. J., Israeli, A. L., \& Antony, M. M. (2004). Is perfectionism good, bad, or both? Examining models of the perfectionism construct. Personality and Individual Differences, 36, 1373-1385.

Bieling, P.J., Israeli, A., Smith, J., \& Antony, M.M. (2003). Making the grade: The behavioural consequences of perfectionism in the classroom. Personality and Individual Differences, $35,163-178$. 
Bieling, P. J., Summerfeldt, L. J., Israeli, A. L., \& Antony, M. M. (2004). Perfectionism as an explanatory construct in comorbidity of Axis I disorders. Journal of Psychopathology and Behavioral Assessment, 26, 193-201.

Blatt, S. J., Zuroff, D. C., Bondi, C. M., Sanislow, C. A. 3RD, \& Pilkonis, P.A. (1998). When and how perfectionism impedes the brief treatment of depression: Further analyses of the National Institute of Mental Health Treatment of Depression Collaborative Research Program. Journal of Consulting and Clinical Psychology, 66, 423-428.

Boone, L., Soenens, B., Vansteenkiste, M., \& Braet, C. (2012). Is there a perfectionist in each of us? An experimental study on perfectionism and eating disorder symptoms. Appetite, 59, 531-540.

Borrelli, B., \& Mermelstein, R. (1994). Goal-setting and behavior change in a smoking cessation program. Cognitive Therapy and Research, 18, 69-83.

Boutelle, K. N., \& Kirschenbaum, D. S. (1998). Further support for consistent self monitoring as a vital component of successful weight control. Obesity Research, 6, 219-224.

Boutelle, K. N., Kirschenbaum, D. S., Baker, R. C., \& Mitchell, M. E. (1999). How can obese weight controllers minimize weight gain during the high risk holiday season? By selfmonitoring very consistently. Health Psychology, 18, 364-368.

Brown, E. J., Heimberg, R. G., Frost, R. O., Makris, G. S., Juster, H. R., \& Leung, A. W. (1999). Relationship of perfectionism to affect, expectations, attributions and performance in the classroom. Journal of Social and Clinical Psychology, 18, 98-120.

Burke, L. E., Sereika, S. M., Music, E., Warziski, M., Styn, M. A., \& Stone, A. A. (2008). Using instrumented paper diaries to document self-monitoring patterns in weight-loss.

Contemporary Clinical Trials, 29, 182-193. 
Burke, L. E., Styn, M. A., Glanz, L. J. Ewing, L. J., Elci, O. U., Conroy, M. B., ... \& Keating, A. L. (2009) SMART Trial: A randomized clinical trial of self-monitoring in behavioral weight management design and baseline findings. Contemporary Clinical Trials, 30, 540551.

Burke, L. E., Wang, J., \& Sevick, M. A. (2011). Self-monitoring in weight-loss: A systematic review of the literature. Journal of the American Dietetic Association, 111, 91-102.

Burns, D. D. (1980, November). The perfectionist's script for self-defeat. Psychology Today, 14, 34-52.

Byrne, S. M. (2002). Psychological aspects of weight maintenance and relapse in obesity. Journal of Psychosomatic Research, 53, 1029-1036.

Carter, M. C., Burley, V. J., Nukjaer, C., \& Cade, J. E. (2013). Adherence to a smartphone application for weight-loss compared to website and paper diary: Pilot randomized controlled trial. Journal of Medical Internet Research, 15, 56-72.

Castro-Fornieles, J., Gual, P., Lahortiga, F., Gila, A., Casulà, V., Fuhrmann, C., . . Toro, J. (2007). Self-oriented perfectionism in eating disorders. International Journal of Eating Disorders, 40, 562-568.

Chang, E. C., \& Sanna, L. J. (2012). Evidence for the validity of the clinical perfectionism questionnaire in a nonclinical population: More than just negative affectivity. Journal of Personality Assessment, 94, 102-108.

Cho, H. (2007). Influences of self-monitoring and goal-setting on drinking refusal self-efficacy and drinking behavior. Alcoholism Treatment Quarterly, 25, 53-65. 
Clara, I. P., Cox, B. J., \& Enns, M. W. (2001). Confirmatory factor analysis of the Depression Anxiety Stress Scales in depressed and anxious patients. Journal of Psychopathology and Behavioral Assessment, 23, 61-67.

Cohen, J. (1992). A power primer. Psychological Bulletin, 112, 155-159.

Conn, V. S. (1998). Older women: Social cognitive theory correlates of health behavior. Women and Health, 26, 71-85.

Cooper, Z., \& Fairburn, C. (1987). The eating disorder examination: A semi-structured interview for the assessment of the specific psychopathology of eating disorders. International Journal of Eating Disorders, 6, 1-8.

De Vet, E., Nelissen, R. M. A., Zeelenberg, M., \& De Ridder, D. T. D. (2012). Ain’t no mountain high enough? Setting high weight-loss goals predict effort and short-term weight-loss. Journal of Health Psychology, 18, 638-647.

De Walt, D. A., Davis, T. C., Wallace, A. S., Seligman, H. K., Bryant-Shilliday, B., Arnold, C. L., .. . Schillinger, D. (2009). Goal-setting in diabetes self-management: Taking the baby steps to success. Patient Education and Counseling, 77, 218-223.

Dennis, K. E., \& Goldberg, A. P. (1996). Weight control self-efficacy types and transitions affect weight-loss outcomes in obese women. Addictive Behaviors, 21, 103-116.

Dickie, L., Surgenor, L. J., Wilson, M., \& McDowall, J. (2012). The structure and reliability of the clinical perfectionism questionnaire. Personality and Individual Differences, 52, 865869.

Dunkley, D. M., Blankstein, K. R., Halsall, J., Williams, M., \& Winkworth, G. (2000). The relation between perfectionism and distress: Hassles, coping, and perceived social support as mediators and moderators. Journal of Counseling Psychology, 47, 437-453. 
Dunkley, D. M., Blankstein, K. R., Masheb, R. M., \& Grilo, C. M. (2006). Personal standards and evaluative concerns dimensions of "clinical" perfectionism: A reply to Shafran et al. (2002, 2003) and Hewitt et al. (2003). Behaviour Research and Therapy, 44, 63-84.

Egan, S. J., Dick, M., \& Allen, P. J. (2012). An experimental investigation of standard setting in clinical perfectionism. Behaviour Change, 29, 183-195.

Egan, S. J., \& Hine, P. (2008). Cognitive behavioural treatment of perfectionism: A single case experimental design series. Behaviour Change, 25, 245-258.

Egan, S. J., Shafran, R., Lee, M., Fairburn, C. G., Cooper, Z., Doll, H. A., . . Watson, H. J. (2016). The reliability and validity of the Clinical Perfectionism Questionnaire in eating disorder and community samples. Behavioural and Cognitive Psychotherapy, 44, 79-91.

Egan, S. J., van Noort, E., Chee, A., Kane, R. T., Hoiles, K. J., Shafran, R., \& Wade, T. D. (2014). A randomized controlled trial of individual face to face versus pure online self-help cognitive behavioural treatment for perfectionism. Behaviour Research and Therapy, 63, 107-113.

Egan, S. J., Wade, T. D., \& Shafran, R. (2011). Perfectionism as a transdiagnostic process: A clinical review. Clinical Psychology Review, 31, 203-212.

Egan, S. J., Wade, T. D., Shafran, R., \& Antony, M. M. (2014). Cognitive-behavioral treatment of perfectionism. New York, NY: Guilford Press.

Elfhag, K. and Rossner, S. (2005) Who succeeds in maintaining weight-loss? A conceptual review of factors associated with weight-loss maintenance and weight regain. Obesity Reviews, 6, 67-85.

Enns, M. W., \& Cox, B. J. (2005). Perfectionism, stressful life events, and the 1-year outcome of depression. Cognitive Therapy and Research, 29, 541-553. 
Fairburn, C. G. (2013). Overcoming binge eating ( $2^{\text {nd }}$ ed.). New York, NY: Guilford Press.

Fairburn, C. G., \& Bèglin, S. J. (1994). Assessment of eating disorders: Interview or self-report questionnaire? International Journal of Eating Disorders, 16, 363-370.

Fairburn, C. G., Cooper, Z., \& Shafran, R. (2003a). Clinical Perfectionism Questionnaire. Unpublished scale, University of Oxford, Oxford, UK.

Fairburn, C. G., Cooper, Z., \& Shafran, R. (2003b). Cognitive behaviour therapy for eating disorders: A "transdiagnostic" theory and treatment. Behaviour Research and Therapy, 41, 509-528.

Fernandez, R., Rajaratnam, R., Evans, K., \& Speizer, A. (2012). Goal-setting in cardiac rehabilitation: Implications for clinical practice. Contemporary Nurse, 43, 13-21.

Flett, G. L., Hewitt, P. L., Blankstein, K. R., \& Mosher, S. W. (1995). Perfectionism, life events, and depressive symptoms: A test of a diathesis-stress model. Current Psychology: A Journal for Diverse Perspectives on Diverse Psychological Issues, 14, 112-137.

Frost, R. O., Heimberg, R. G., Holt, C. S., Mattia, J. I., \& Neubauer, A. L. (1993). A comparison of two measures of perfectionism. Personality and Individual Differences, 14, 119-126.

Frost, R. O., Martin, P., Lahart, C., \& Rosenblate, R. (1990). The dimensions of perfectionism. Cognitive Therapy and Research, 14, 449-468.

Ganske, K., \& Ashby, J. S. (2007). Multidimensional perfectionism and career decision making self-efficacy. Journal of Employment Counselling, 44, 17-28.

Glaser, B. and Strauss, A. (1967). The Discovery of Grounded Theory. Chicago, IL: Aldine.

Glover, D. S., Brown, G. P., Fairburn, C. G., \& Shafran, R. (2007). A preliminary evaluation of cognitive-behaviour therapy for clinical perfectionism: A case series. British Journal of Clinical Psychology, 46, 85-94. 
Glynn, S. M., \& Ruderman, A. J. (1986). The development and validation of an eating selfefficacy scale. Cognitive Therapy and Research, 10, 403-420.

Gormally, J., Rardin, D., \& Black, S. (1980). Correlates of successful response to a behavioral weight control clinic. Journal of Counseling Psychology, 27, 179-191.

Gosselin, J. T. (2003). Perfectionism: Examination from a self-regulation framework (Doctoral dissertation). Retrieved from PsycINFO. (Order No. AAI3079323)

Gosselin, J. T., Boone, A. L., Sinek, D., \& Tangney, J. P. (2002, February). A new measurement of perfectionism: Capturing the maladaptive nature of perfectionism where other measures have failed. Poster presented at the meeting of the Society for Personality and Social Psychology, Savannah, GA.

Haapala, I., Barengo, N. C., Biggs, S., Surakka, L., \& Manninen, P. (2009). Weight-loss by mobile phone: A 1-year effectiveness study. Public Health Nutrition, 12, 2382-2391.

Hamacheck, D. E. (1978). Psychodynamics of normal and neurotic perfectionism, Journal of Human Behavior, 15, 27-33.

Hart, B. A., Gilner, F. H., Handal, P. J., \& Gfeller, J. D. (1998). The relationship between perfectionism and self-efficacy. Personality and Individual Differences, 24, 109-113.

Hewitt, P. L., \& Flett, G. L. (1991). Perfectionism in the self and social contexts: Conceptualization, assessment, and association with psychopathology. Journal of Personality and Social Psychology, 60, 456-470.

Hewitt, P. L., Flett, G. L., Besser, A., Sherry, S. B., \& McGee, B. (2003). Perfectionism is multidimensional: A reply to Shafran, Cooper and Fairburn (2002). Behaviour Research and Therapy, 41, 1221-1236. 
Hewit, P. L., Flett, G. L., Turnbull-Donovan, W., \& Mikail, S. F. (1991). The Multidimensional Perfectionism Scale: Reliability, validity, and psychometric properties in psychiatric samples. Journal of Consulting and Clinical Psychology, 3, 464-468.

Hoenig, J. M., \& D. M. Heisey. (2001). The abuse of power: The pervasive fallacy of power calculations in data analysis. The American Statistician, 55, 19-24.

Hoiles, K., Egan, S. J., Kane, R. T., \& Rees, C. S. (2014). A randomized controlled trial of guided self-help treatment for clinical perfectionism. Manuscript in preparation.

Hrabosky, J. I., White, M. A., Masheb, R. M., Rothschild, B. S., Burke-Martindale, C., \& Grilo, C. M. (2008). Psychometric evaluation of the eating disorder examination-questionnaire for bariatric surgery candidates. Obesity, 16, 763-769.

Iketani, T., Kiriike, N., Stein, M. B., Nagao, K., Nagata, T., Minamikawa, N., . . Fukuhara, H. (2002). Relationship between perfectionism and agoraphobia in patients with panic disorder. Cognitive Behaviour Therapy, 31, 119-128.

Irwin, C. A. (1998). Eating self-efficacy among adults in behavioral, diet, and exercise based weight control programs (Doctoral dissertation). Retrieved from PsycINFO (Order No. AAM9810026)

Jeffery, R. W., Bjornson-Benson, W. M., Rosenthal, B. S., Lindquist, R. A., Kurth, C. L., \& Johnson, S. L. (1984). Correlates of weight-loss and its maintenance over two years of follow-up among middle-aged men. Preventive Medicine, 13, 155-168.

Jensen M. D., Ryan D. H., Apovian C. M., Ard J. D., Comuzzie A. G., Donato K. A., ... \& Yanovski S. Z. (2014). 2013 AHA/ACC/TOS guideline for the management of overweight and obesity in adults: A report of the American College of Cardiology/American Heart 
Association Task Force on Practice Guidelines and The Obesity Society. Journal of the American College of Cardiology, 63, 2986-3023.

Johnson, F., \& Wardle, J. (2005). Dietary restraint, body dissatisfaction, and psychological distress: A prospective analysis. Journal of Abnormal Psychology, 114, 119-125.

Juster, H. R., Heimberg, R. G., Frost, R. O., Holt, C. S., Mattia, J. I., \& Faccenda, K. (1996). Social phobia and perfectionism. Personality and Individual Differences, 21, 403-410.

Kitsantas, A. (2000) The role of self-regulation strategies and self-efficacy perceptions in successful weight-loss maintenance. Psychology and Health, 15, 811-820.

Kobori, O., Hayakawa, M., \& Tanno, Y. (2009). Do perfectionists raise their standards after success? An experimental examination of the revaluation of standard setting in perfectionism. Journal of Behavior Therapy and Experimental Psychiatry, 40, 515-521.

Kralj, D. (1989). Perfectionism, self-efficacy, and self-control as predictors of diet success (Doctoral dissertation). Retrieved from PsycINFO. (Order No. 3626694)

Krause, K. L., \& Antony, M. M., \& Cassin, S. E. (2016, October). Clinical perfectionism predicts raising the bar: Validation of a new questionnaire. Poster presented at the meeting of the Association for Behavioral and Cognitive Therapies, New York, NY.

Kubzansky, L., Gilthorpe, M., \& Goodman, E. (2012). A prospective study of psychological distress and weight status in adolescents/young adults. Annals of Behavioral Medicine, 43, 219-228.

Kyllo, L. B., \& Landers, D. M. (1995). Goal-setting in sport and exercise: A research synthesis to resolve the controversy. Journal of Sport and Exercise Psychology, 17, 117-137.

Laliberté, M., McCabe, R. E., \& Taylor, V. (2009). The cognitive behavioral workbook for weight management: A step-by-step program. Oakland, CA: New Harbinger Publications. 
RAISING THE BAR

Laurenti, H. J., Bruch, M. A., \& Haase, R. F. (2008). Social anxiety and socially prescribed perfectionism: Unique and interactive relationships with maladaptive appraisal of interpersonal situations. Personality and Individual Differences, 45, 55-61.

Lee, W., Chae, Y. M., Kim, S., Ho, S. H., \& Choi, I. (2010). Evaluation of a mobile phone-based diet game for weight control. Journal of Telemedicine and Telecare, 16, 270-275.

Lenth, R. V. (2007a). Post hoc power: Tables and commentary. Department of Statistics and Actuarial Science Technical Report No. 378. Iowa City, IA.

Lenth, R. V. (2007b). Statistical power calculations. Journal of Animal Science, 85(E. Suppl.): E24-E29.

Levy, R. L., Finch, E. A., Crowell, M. D., Talley, N. J., \& Jeffery R. W. (2007). Behavioral intervention for the treatment of obesity: Strategies and effectiveness data. American Journal of Gastroenterology, 102, 2314-2321.

Lo, A., \& Abbott, M. J. (2013a). The impact of manipulating expected standards of performance for adaptive, maladaptive, and non-perfectionists. Cognitive Therapy and Research, 37, $762-778$.

Lo, A., \& Abbott, M. J. (2013b). Review of the theoretical, empirical, and clinical status of adaptive and maladaptive perfectionism. Behaviour Change, 30, 96-116.

LoCicero, K., \& Ashby, J. S. (2000). Multidimensional perfectionism and self-reported selfefficacy in college students. Journal of College Student Psychotherapy, 15, 47-56.

Locke, E. A., \& Latham, G. P. (1990) A theory of goal-setting and task performance. Englewood Cliffs, NJ: Prentice-Hall.

Locke, E. A., \& Latham, G. P. (2002). Building a practically useful theory of goal-setting and task motivation: A 35-year odyssey. American Psychologist, 57, 705-717. 
RAISING THE BAR

Lovibond, P. F., \& Lovibond, S. H. (1995a). The structure of negative emotional states:

Comparison of the Depression Anxiety Stress Scales (DASS) with the Beck Depression and Anxiety Inventories. Behaviour Research and Therapy, 33, 335-343.

Lovibond, S.H. \& Lovibond, P.F. (1995b). Manual for the Depression Anxiety Stress Scales. (2nd Ed.). Sydney, Australia: Psychology Foundation.

Martin, T. R., Flett, G.L., Hewitt, P. L., Krames, L., \& Szanto, G. (1996). Personality correlates of depression and healthy symptoms: A test of a self-regulation model. Journal of Research in Personality, 31, 264-277.

Mattila, E., Lappalainen, R., Pärkkä, J., Salminen, J., \& Korhonen, 1. (2010). Use of a mobile phone diary for observing weight management and related behaviours. Journal of Telemedicine and Telecare, 16, 260-264.

McCann, B. S., Bovbjerg, V. E., Brief, D. J., Turner, C., Follette, W. C., Fitzpatrick, V., .. . \& Knopp, R. H. (1995). Relationship of self-efficacy to cholesterol lowering and dietary change in hyperlipidemia. Annals of Behavioral Medicine, 17, 221-226.

McKee, H., Ntoumanis, N., \& Smith, B. (2013). Weight maintenance: Self-regulatory factors underpinning success and failure. Psychology and Health, 28, 1207-1223.

Michie, S., Abraham, C., Whittington, C., McAteer, J., \& Gupta, S. (2009). Effective techniques in healthy eating and physical activity interventions: A meta-regression. Health Psychology, 28, 690-701.

Middelweerd, A., Mollee, J. S., van der Wal, C. N., Brug, J., \& te Velde, S. J. (2014). Apps to promote physical activity among adults: A review and content analysis. International Journal of Behavioral Nutrition and Physical Activity, 11, 1-9. 
Mills, J., \& Blankstein, K. R. (2000). Perfectionism, intrinsic vs extrinsic motivation, and motivated strategies for learning: A multidimensional analysis of university students. Personality and Individual Differences, 29, 1191-1204.

Mischel, W. (1968). Personality and assessment. New York: Wiley.

Mischel, W. (2009). From personality and assessment (1968) to personality science, 2009. Journal of Research in Personality, 43, 282-290.

Mischel, W. (1973). Toward a cognitive social learning reconceptualization of personality. Psychological Review, 80, 252-283.

Mischel, W., \& Peake, P. K. (1982). Beyond déjà vu in the search for cross-situational consistency. Psychological Review, 89, 730-755.

Mischel, W., \& Shoda, Y. (1995). A cognitive-affective system theory of personality: Reconceptualizing situations, dispositions, dynamics, and invariance in personality structure. Psychological Review, 102, 246-268.

Moretz, M. W., \& McKay, D. (2009). The role of perfectionism in obsessive-compulsive symptoms: "Not just right" experiences and checking compulsions. Journal of Anxiety Disorders, 23, 640-644.

Napolitano, M. A., Hayes, S., Bennett, G. G., Ives, A. K., \& Foster, G. D. (2013). Using Facebook and text messaging to deliver a weight-loss program to college students. Obesity, 21, 25-31.

Obsessive Compulsive Cognitions Working Group (1997). Cognitive assessment of obsessivecompulsive disorder. Behaviour Research and Therapy, 35, 667-681.

Otani, K., \& Sakurai, S. (1995). Relationship of perfectionism to depression and hopelessness in college students. Japanese Journal of Psychology, 66, 41-47. 
Pearson, E. S. (2012). Goal-setting as a health behavior change strategy in overweight and obese adults: A systematic literature review examining intervention components. Patient Education and Counseling, 87, 32-42.

Peters, G. A. (1996). An investigation of psychosocial factors associated with weight-loss maintenance among females (Doctoral dissertation). Retrieved from PsycINFO (Order No. AAM9541285)

Peterson, C. B., Crosby, R. D., Wonderlich, S. A., Joiner, T., Crow, S. J., Mitchell, J. E., . . le Grange, D. (2007). Psychometric properties of the eating disorder examinationquestionnaire: Factor structure and internal consistency. International Journal of Eating Disorders, 40, 386-389.

Prezioso, M. (1995). Social learning variables as predictors of success in a behavioral weightloss program (Doctoral dissertation). Retrieved from PsycINFO (Order No. AAM9511241)

Rice, K. G., \& Ashby, J. S. (2007). An efficient method for classifying perfectionists. Journal of Counseling Psychology, 54, 72-85.

Rice, K. G., Ashby, J. S., \& Slaney, R. B. (1998). Self-esteem as a mediator between perfectionism and depression: A structural equation analysis. Journal of Counseling Psychology, 45, 304-314.

Riley, C., Lee, M., Cooper, Z., Fairburn, C. \& Shafran, R. (2007). A randomized controlled trial of cognitive-behaviour therapy for clinical perfectionism: A preliminary study. Behaviour Research and Therapy, 45, 2221-2231.

Riley, C., \& Shafran, R. (2005). Clinical perfectionism: A preliminary qualitative analysis. Behavioural and Cognitive Psychotherapy, 33, 369-374. 
Rodin, J., Elias, M., Silberstein, L. R., \& Wagner, A. (1988). Combined behavioral and pharmacologic treatment for obesity: Predictors of successful weight maintenance. Journal of Consulting and Clinical Psychology, 56, 399-404.

Rosen, J. C., Tacy, B., \& Howell, D. (1990). Life stress, psychological symptoms and weight reducing behavior in adolescent girls: A prospective analysis. International Journal of Eating Disorders, 9, 17-26.

Schnoll, R. \& Zimmerman, B. J. (2001). Self-regulation training enhances dietary self-efficacy and dietary fiber consumption. Journal of the American Dietetic Association, 101, 10061011

Shafran, R., Cooper, Z. \& Fairburn, C.G. (2002). Clinical perfectionism: A cognitivebehavioural analysis. Behaviour Research and Therapy, 40, 773-791.

Shafran, R., Cooper, Z., \& Fairburn, C. G. (2003). “Clinical perfectionism” is not “multidimensional perfectionism": A reply to Hewitt, Flett, Besser, Sherry, \& McGee. Behaviour Research and Therapy 41, 1217-1220.

Shafran, R., Egan, S. J., \& Wade, T. D. (2010). Overcoming perfectionism: A self-help guide using cognitive-behavioural techniques. London, UK: Constable \& Robinson.

Shafran, R., Lee, M., \& Fairburn, C. G. (2004). Clinical perfectionism: A case report. Behavioural and Cognitive Psychotherapy, 32, 353-357.

Shafran, R., Lee, M., Payne, E., \& Fairburn, C. G. (2006). The impact of manipulating personal standards on eating attitudes and behaviour. Behaviour Research and Therapy, 44, 897906.

Shay, L. E., Seibert, D., Watts, D., Sbrocco, T., \& Pagliara, C. (2009). Adherence and weightloss outcomes associated with food-exercise diary preference in a military weight 
RAISING THE BAR

management program. Eating Behaviors, 10, 220-227.

Sinclair, S. J., Siefert, C. J., Slavin-Mulford, J., Stein, M. B., Renna, M., \& Blais, M. A. (2012).

Psychometric evaluation and normative data for the Depression, Anxiety, and Stress

Scales-21 (DASS-21) in a nonclinical sample of U.S. adults. Evaluation and the Health Professions, 35, 259-279.

Slaney, R. B., Rice, K. G., Mobley, M., Trippi, J., \& Ashby, J. S. (2001). The Revised Almost Perfect Scale. Measurement and Evaluation in Counselling and Development, 34, 130145.

Steele, A. L., Waite, S., Egan, S. J., Finnigan, J., Handley, A. \& Wade, T. D. (2013) Psychoeducation and group cognitive-behavioural therapy for clinical perfectionism: A case-series evaluation. Behavioural and Cognitive Psychotherapy, 41, 129-143.

Stoeber, J. (2014). How other-oriented perfectionism differs from self-oriented and socially prescribed perfectionism. Journal of Psychopathology and Behavioral Assessment, 36, 329-338.

Stoeber, J. (2015). How other-oriented perfectionism differs from self-oriented and socially prescribed perfectionism. Journal of Psychopathology and Behavioral Assessment, 37, 611-623.

Stoeber, J., \& Damian, L. E. (2014). The Clinical Perfectionism Questionnaire: Further evidence for two factors capturing perfectionistic strivings and concerns. Personality and Individual Differences, 61-62, 38-42.

Stoeber, J., Hutchfield, J., \& Wood, K.V. (2008). Perfectionism, self-efficacy, and aspiration level: Differential effects of perfectionistic striving and self-criticism after success and failure. Personality and Individual Differences, 45, 323-327. 
Stoeber, J., \& Otto, K. (2006). Positive conceptions of perfectionism: Approaches, evidence, challenges. Personality and Social Psychology Review, 10, 295-319.

Stoeber, J., Otto, K., Pescheck, E., Becker, C., \& Stoll, O. (2007). Perfectionism and competitive anxiety in athletes: Differentiating striving for perfection and negative reactions to imperfection. Personality and Individual Differences, 42, 959-969.

Stotland, S., \& Zuroff, D. C. (1991). Relations between multiple measures of dieting selfefficacy and weight change in a behavioral weight control program. Behavior Therapy, 22, 47-59.

Strecher, V. J., DeVellis, B. M., Becker, M. H., \& Rosenstock, I. M. (1986). The role of selfefficacy in achieving health behavior change. Health Education Quarterly, 13, 73-92.

Tabachnick, B. G., \& Fidell, L. S. (2007). Using multivariate statistics (5th ed.). Boston, MA: Allyn and Bacon.

Teixeira, P. J., Going, S. B., Houtkooper, L. B., Cussler, E. C., Metcalfe, L. L., Blew, R. M., ... \& Lohman, T. G. (2004). Pretreatment predictors of attrition and successful weight management in women. International Journal of Obesity, 28, 1124-1133.

Telch, C. F., Pratt, E. M., \& Niego, S. H. (1998). Obese women with binge eating disorder define the term binge. International Journal of Eating Disorders, 24, 313-317.

Terry-Short, L., Glynn Owens, R., Slade, P. D., \& Dewey, M. E. (1995). Positive and negative perfectionism. Personality and Individual Differences, 18, 663-668.

Tsai, C. C., Lee, G., Raab, F., Norman, G. J., Sohn, T., Griswold, W. G., \& Patrick, K. (2007). Usability and feasibility of PmEB: A mobile phone application for monitoring real time caloric balance. Mobile Networks and Applications, 12, 173-184. 
Wharton, C. M., Johnston, C. S., Cunningham, B. K., \& Sterner, D. (2014). Dietary selfmonitoring, but not dietary quality, improves with use of smartphone app technology in an 8-week weight-loss trial. Journal of Nutrition Education and Behavior, 46, 440-444.

World Health Organization (WHO, 2006). BMI Classification. Retrieved from http://apps.who.int/bmi/index.jsp?introPage=intro_3.html

Yon, B. A., Johnson, R. K., Harvey-Berino, J., Gold, B. C., \& Howard, A. B. (2007). Personal digital assistants are comparable to traditional diaries for dietary self-monitoring during a weight-loss program. Journal of Behavioral Medicine, 30, 165-175.

Zhang, B., \& Cai, T. (2012). Moderating effects of self-efficacy in the relations of perfectionism and depression. Studia Psychologica, 54, 15-21.

Zhang, B., Xie, J., \& Cai, T. (2013). Construct and characteristics of perfectionism. Chinese Mental Health Journal, 27, 215-219.

Zuroff, D. C., Blatt, S. J., Sotsky, S. M., Krupnick, J. L., Martin, D. J., Sanislow, C. A., \& Simmens, S. (2000). Relation of therapeutic alliance and perfectionism to outcome in brief outpatient treatment of depression. Journal of Consulting and Clinical Psychology, 68, $114-124$ 